\title{
Validation of EIT using Simultaneous Dynamic CT images
}

\author{
By \\ Hassan A. Hannoon, BEng \\ A thesis submitted to the \\ Faculty of Graduate Studies and Postdoctoral affairs \\ in partial fulfillment of the requirements for \\ the degree of Master of Applied Science in Biomedical Engineering \\ Ottawa-Carleton Institute for Biomedical Engineering (OCIBME) \\ Department of Systems and Computer Engineering \\ Carleton University \\ Ottawa, Ontario, Canada, K1S 5B6 \\ January, 2011
}

(C) Copyright, Hassan Hannoon 
Library and Archives

Canada

Published Heritage Branch

395 Wellington Street

Ottawa ON K1A ON4

Canada
Bibliothèque et

Archives Canada

Direction du

Patrimoine de l'édition

395 , rue Wellington

Ottawa ON K1A ON4

Canada
Your file Votre reférence

ISBN: 978-0-494-79534-7

Our file Notre référence

ISBN: 978-0-494-79534-7
NOTICE:

The author has granted a nonexclusive license allowing Library and Archives Canada to reproduce, publish, archive, preserve, conserve, communicate to the public by telecommunication or on the Internet, loan, distribute and sell theses worldwide, for commercial or noncommercial purposes, in microform, paper, electronic and/or any other formats.

The author retains copyright ownership and moral rights in this thesis. Neither the thesis nor substantial extracts from it may be printed or otherwise reproduced without the author's permission.
AVIS:

L'auteur a accordé une licence non exclusive permettant à la Bibliothèque et Archives Canada de reproduire, publier, archiver, sauvegarder, conserver, transmettre au public par télécommunication ou par l'Internet, prêter, distribuer et vendre des thèses partout dans le monde, à des fins commerciales ou autres, sur support microforme, papier, électronique et/ou autres formats.

L'auteur conserve la propriété du droit d'auteur et des droits moraux qui protège cette thèse. $\mathrm{Ni}$ la thèse ni des extraits substantiels de celle-ci ne doivent être imprimés ou autrement reproduits sans son autorisation.
In compliance with the Canadian Privacy Act some supporting forms may have been removed from this thesis.

While these forms may be included in the document page count, their removal does not represent any loss of content from the thesis.
Conformément à la loi canadienne sur la protection de la vie privée, quelques formulaires secondaires ont été enlevés de cette thèse.

Bien que ces formulaires aient inclus dans la pagination, il n'y aura aucun contenu manquant. 
To my family 


\begin{abstract}
Electrical impedance tomography (EIT) estimates a cross-sectional conductivity image of a body from surface electrical measurements. One promising application of EIT is for monitoring lung ventilation to help optimize management of intensive care patients. To test the applicability of EIT for this application, it is important to validate EIT images to understanding of the magnitude and types of errors. This thesis seeks to develop a set of software tools to validate regional EIT against simultaneous dynamic CT. The implemented algorithm analyzes data obtained from each image and estimates research parameters to detect the accuracy of EIT measurements. Data were derived from an experimental lung injury model, created by repeated lavage in pigs. Lungs were inflated by constant slow flow inflation while dynamic CT and EIT data were simultaneously acquired. Data were obtained during the inflation and deflation phase before and after experimental lung injury. Regions of interest (ROI) were selected on both EIT and CT images, and data were analyzed and compared based on five parameters of the image magnitude and temporal behaviour. Of the eleven pigs studied and analyzed, six pigs' data were excluded. The five research parameters were estimated on each image measurements for healthy and acute lung injury (ALI) diseased pigs' data. Regional behaviour of EIT and CT were either uncorrelated or inversely correlated. We hypothesize that this unexpected inverse correlation is due to errors in the positions of reconstructed image ROI in the EIT images. The change in research parameters from healthy to ALI was tested and was significant for some parameters and some animals. EIT gave significant differences more often than CT. These results support a certain confidence in global EIT measures, while suggesting future work to better understand position errors in EIT images.
\end{abstract}




\section{Acknowledgements}

I would like to thank my supervisor Dr. Andy Adler, professor in Carleton University and Canada Research Chair (Tier II) in Biomedical engineering, for his great support, guidance, and encouragement. His experience and suggestions were the main key factors in accomplishing the work done in this thesis.

I would also like to thank Carleton University for having the chance and be one of the graduate students in Biomedical engineering program. Through this opportunity, I have chance to know and work with the EIT lab team, Yasheng Maimaitijiang, Obaydah Jaber, Navid Bahrani, Beyman Tahmati, and Ravi Bhanabhai. 


\section{Table of Contents}

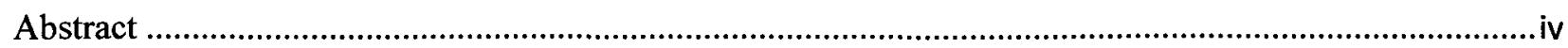

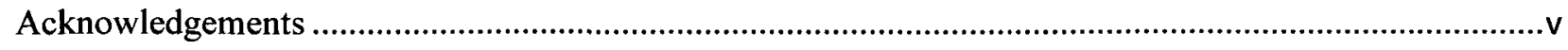

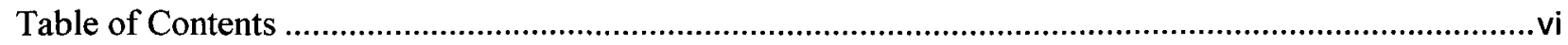

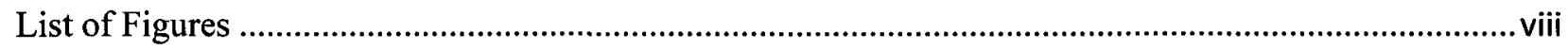

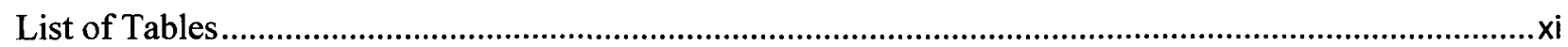

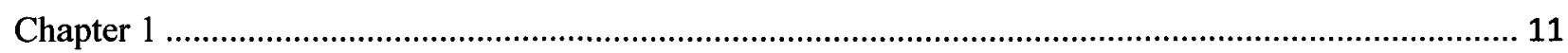

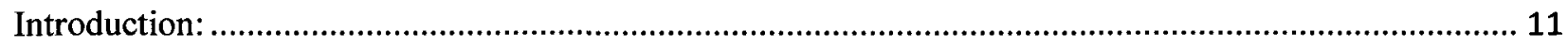

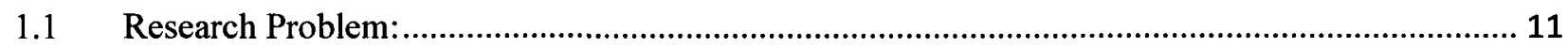

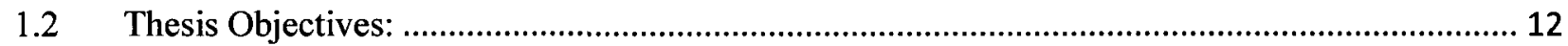

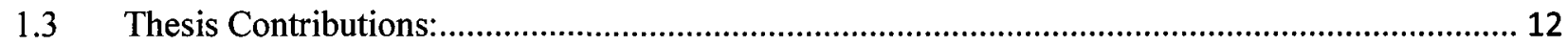

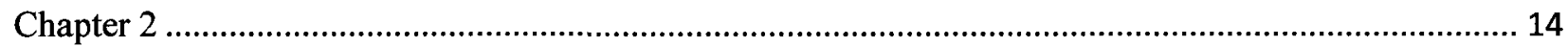

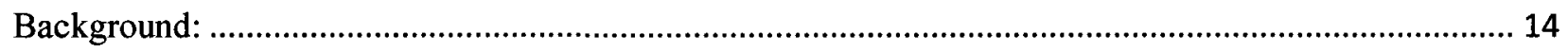

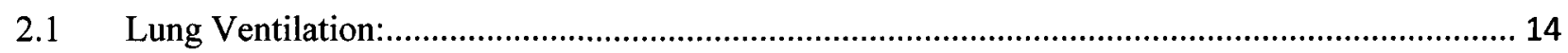

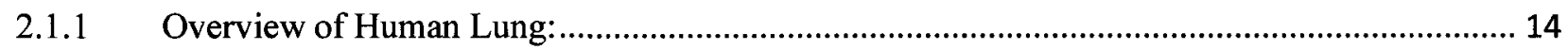

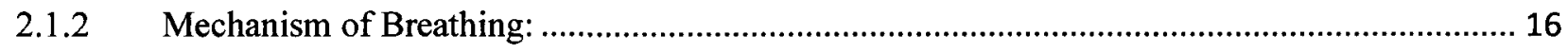

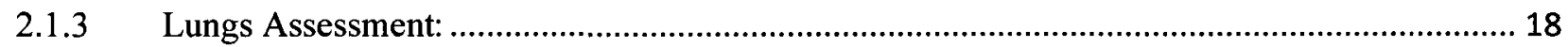

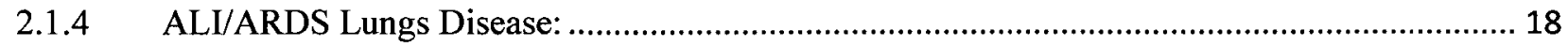

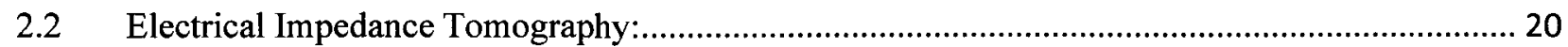

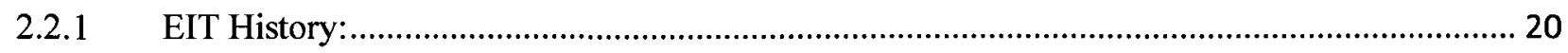

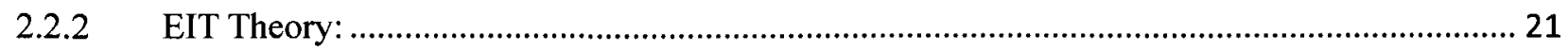

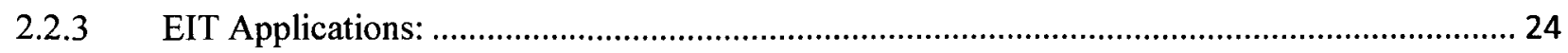

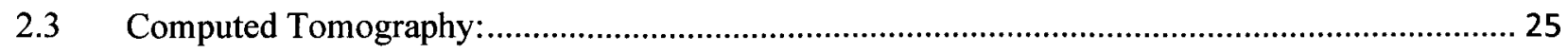

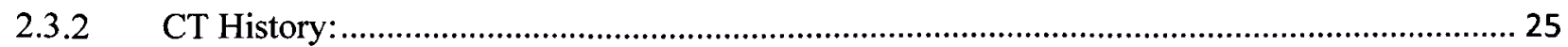

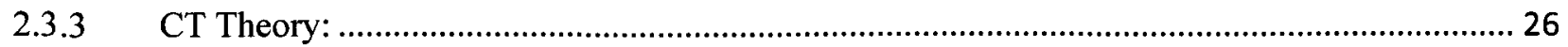




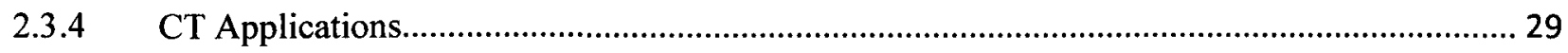

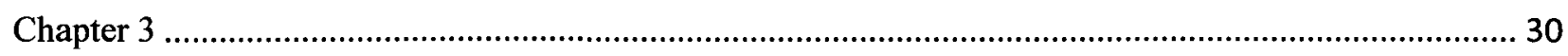

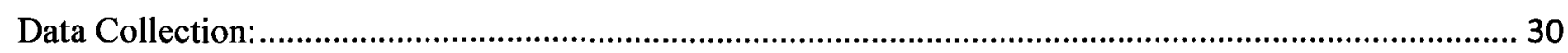

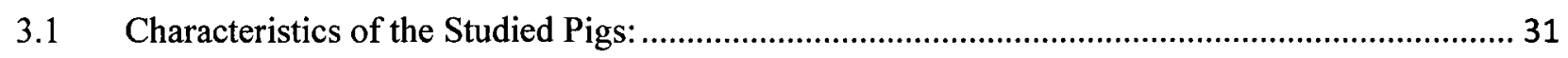

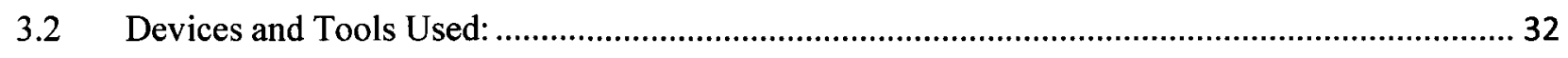

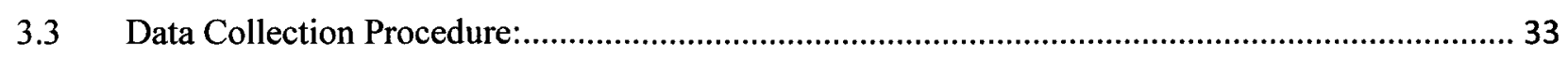

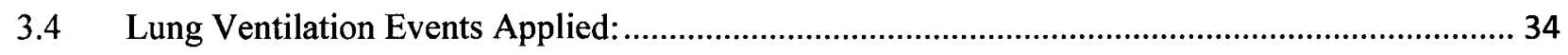

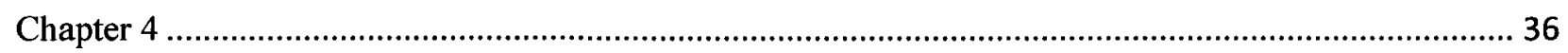

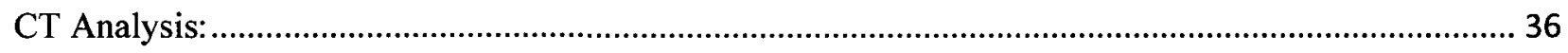

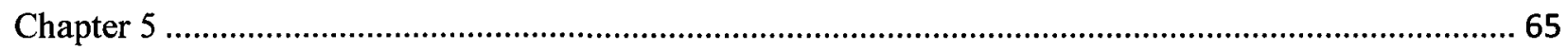

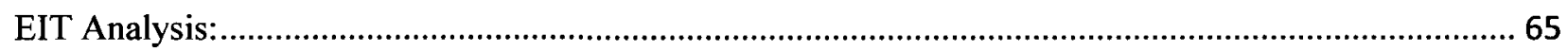

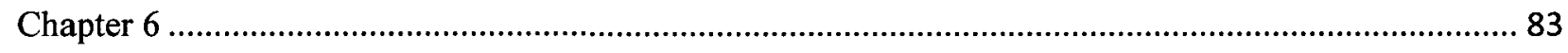

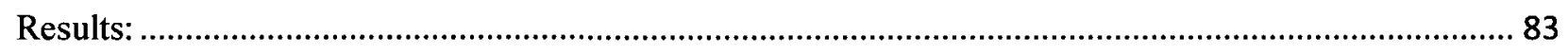

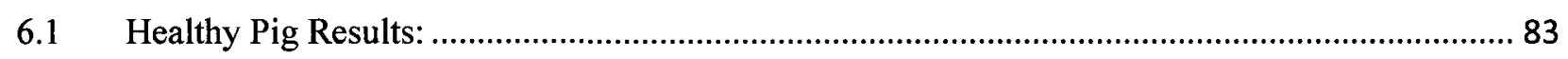

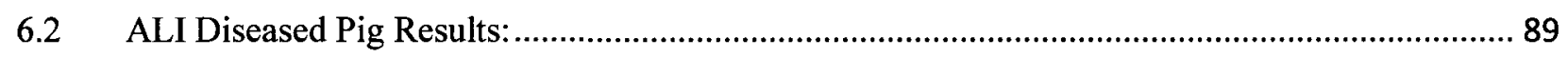

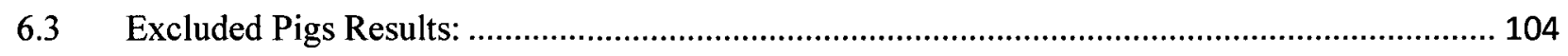

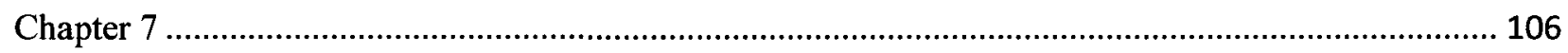

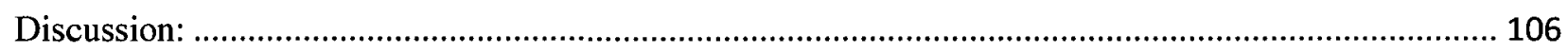

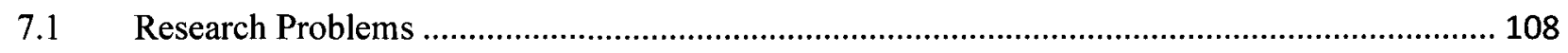

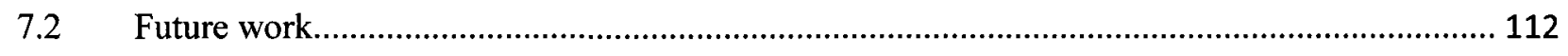

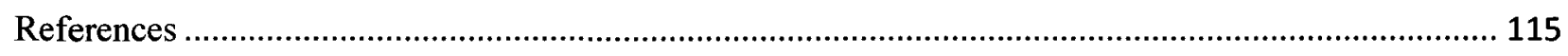




\section{List of Figures}

Figure 2- 1: Basic parts of human respiratory system. .................................................. 15

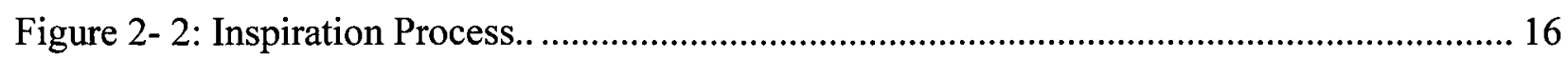

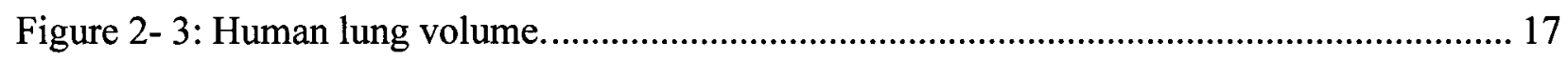

Figure 2- 4: A block diagram of EIT system. ............................................................. 21

Figure 2- 5: EIT thorax phantom. ................................................................................... 23

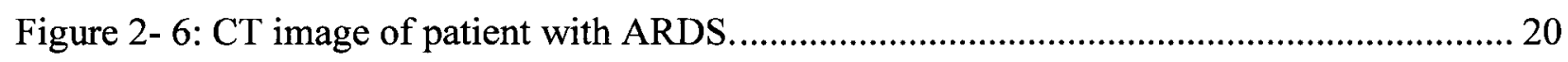

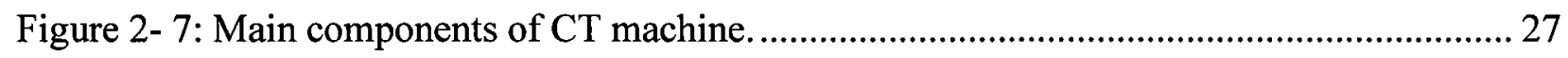

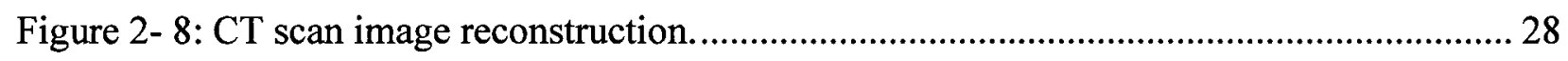

Figure 2- 9: Sample CT cross sectional image of a pig chest......................................... 29

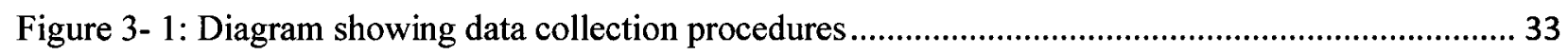

Figure 4- 1: Research project overview to process EIT and CT images. ................................ 36

Figure 4- 2: CT image for a sample healthy pig ............................................................ 37

Figure 4- 3: CT image for a sample healthy pig before and after air filling.......................... 38

Figure 4- 4: CT image for a sample healthy pig before and after air emptying....................... 39

Figure 4- 5: CT image for a sample ALI diseased pig before and after air filling.................. 40

Figure 4- 6: CT image for a sample ALI diseased pig before and after air emptying................ 41

Figure 4- 7: Flowchart illustrating the algorithmic steps implemented to analyse CT data ........ 44

Figure 4- 8: CT image before and after image adjustment step........................................... 44

Figure 4- 9: CT image after applying image thresholding step. ........................................... 46

Figure 4- 10: CT image after applying hole filling step.. ................................................ 47

Figure 4- 11: CT image after applying the main object selection step................................ 48

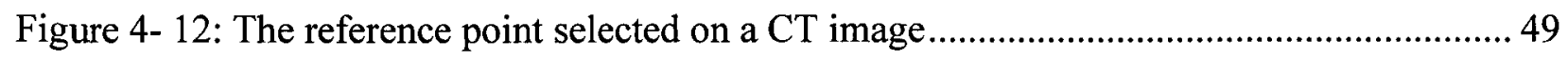

Figure 4- 13: Representative selection of ROIs chosen on CT image................................... 50 
Figure 4- 14: 5 research parameters identified on CT data....................................................... 52

Figure 4- 15: Before and after curve cleaning step for CT data. ................................................. 54

Figure 4- 16: Finding the highest point at air filling on CT data.................................................. 55

Figure 4- 17: Finding the lowest point at air filling on CT data................................................... 56

Figure 4- 18: Finding the highest point at air emptying on CT data............................................. 57

Figure 4- 19: Finding the lowest point at air emptying on CT data.......................................... 58

Figure 4- 20: Estimating research parameter A1 on CT data. ......................................................... 59

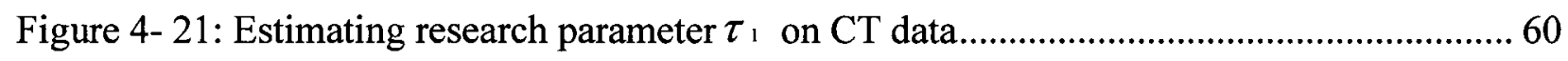

Figure 4- 22: Estimating research parameter A2 on CT data ......................................................... 61

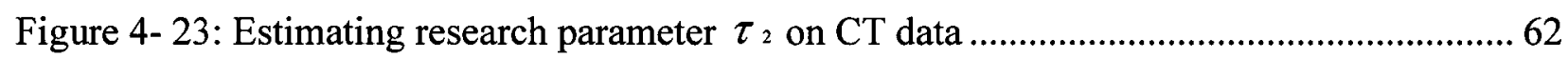

Figure 4- 24: Estimating research parameter slope on CT data ........................................................ 64

Figure 5- 1: EIT images for healthy pig before and after air filling............................................66

Figure 5- 2: EIT images for healthy pig before and after air emptyinging....................................67

Figure 5- 3: EIT images for ALI diseased pig before and after air filling.................................. 68

Figure 5- 4: EIT images for ALI diseased pig before and after air emptying .............................. 69

Figure 5- 5: A flowchart describing the algorithm used to analyse EIT data ............................... 70

Figure 5- 6: Representative selection of (ROI) selected on EIT image ......................................... 71

Figure 5- 7: Before and after curve cleaning step for EIT data. ……........................................ 72

Figure 5- 8: Finding the highest point at air filling on EIT data.................................................. 73

Figure 5- 9: Finding the lowest point at air filling on EIT data....................................................... 74

Figure 5- 10: Finding the highest point at air emptying on EIT data. ......................................... 75

Figure 5- 11: Finding the lowest point at air emptying on EIT data............................................. 76

Figure 5- 12: Estimating research parameter A1 on EIT data. ....................................................... 77

Figure 5- 13: Estimating research parameter $\tau_{1}$ on EIT data.................................................. 78 
Figure 5- 14: Estimating research parameter A2 on EIT data. ……....................................... 79

Figure 5- 15: Estimating research parameter $\tau_{2}$ on EIT data.................................................... 80

Figure 5- 16: Estimating research parameter slope on EIT data...................................................... 81

Figure 6- 1: CT cross sectional image showing the locations of a representative selection......................... 83

Figure 6- 2:EIT image showing the locations of a representative selection................................................. 84

Figure 6- 3: (left) CT image and (right) EIT image of the selected ROI number 40................................. 85

Figure 6- 4: (left) CT graph and (right) EIT graph of the selected ROI number 40..................................... 86

Figure 6- 5: $\mathrm{A} 1$ and $\tau_{1}$ correlation between $\mathrm{CT}$ and EIT images...................................................... 87

Figure 6- 6: $\mathrm{A} 2$ and $\tau_{2}$ correlation between CT and EIT images....................................................... 88

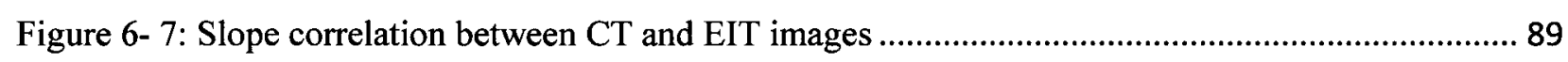

Figure 6- 8: CT and EIT graphs of ALI diseased pig for a selected ROI number 40................................. 90

Figure 6- 9: $\mathrm{Al}$ and $\tau_{1}$ correlation between $\mathrm{CT}$ and EIT images of ALI diseased pig lungs ................... 91

Figure 6- 10: A2 and $\tau_{2}$ correlation between CT and EIT images of ALI diseased pig lungs................. 92

Figure 6- 11: Slope correlation between CT and EIT images of ALI diseased pig images. ....................... 93

Figure 6- 12: $\mathrm{Al}$ and $\tau_{1}$ correlation between healthy and ALI diseased pig lungs images. ..................... 97

Figure 6- 13: A2 and $\tau_{2}$ correlation between healthy and ALI diseased pig lungs images...................... 98

Figure 6- 14: Slope correlation between Healthy and ALI diseased pig lungs images ............................... 99

Figure 6- 15: Excluded data obtained from CT and EIT images....................................................... 104

Figure 7- 1: CT image showing the problem, heart covering the top left lung......................... 109

Figure 7-2: EIT showing the problem, thickness of the boundaries are not clear ..................... 110

Figure 7- 3: EIT image showing the problem, lungs and boundaries size not changing............ 111

Figure 7- 4: CT image showing the lungs and boundaries size changing. ................................ 111

Figure 7- 5: CT image showing noise artifact problem ......................................................... 112 


\section{List of Tables}

Table 3- 1: Characteristics of the 11 studied pigs used for the research experiment................. 31

Table 6- 1: 5 research parameters estimated from both CT and EIT healthy images........................... 86

Table 6- 2: 5 research parameters estimated from both CT and EIT ALI diseased images..................... 90

Table 6- 3: Correlation coefficients for 5 research parameters estimated on healthy and ALI images...... 94

Table 6- 4: Correlation coefficients for the y-coordinate and parameters estimated on EIT images ......... 95

Table 6- 5: Correlation coefficients for the y-coordinate and parameters estimated on CT images .......... 96

Table 6- 6: Correlation coefficients for each research parameter between healthy and ALI diseased..... 100

Table 6- 7: Average change and standard deviation of each parameter between healthy and ALI......... 101

Table 6- 8: P-value showing that healthy parameters are different from ALI diseased parameters......... 102

Table 6- 9: P-value showing that the correlations between healthy and ALI images is not Random...... 103 


\section{Chapter 1}

\section{Introduction:}

Electrical Impedance Tomography (EIT) is an imaging technique used for monitoring regional lungs ventilation and pulmonary disorders (Frerichs et al, 1998, 2003). EIT images the internal structure of a body by measuring the differences of voltages at its boundaries. It can be used as a ventilation monitoring technique to optimize treatments of lungs diseases such as acute lung injury (ALI) and acute respiratory distress syndrome (ARDS). This thesis aims to validate EIT measurements on ventilation versus dynamic Computed Tomography (CT) measurements taken simultaneously on live pigs. Functional regions of interest (ROI) on EIT images are studied along with CT ROI. This thesis focuses on analysis and validation of EIT images versus dynamic CT images with respect to the functional behaviour of regional lungs of some healthy and ALI/ARDS diseased pigs.

\subsection{Research Problem:}

Modern healthcare relies on medical images when need to monitor the physiological changes of such an organ in a body. Treatment diseases (e.g., ALI/ARDS) need a diagnostic technology such as medical images for better assessment. For example, CT images are used to monitor lungs behaviour and detect diseases like ALI/ARDS and lungs cancer.

EIT provides different type of medical images. It produces images representing the distribution of electrical characteristics, conductivity or sensitivity, across a sectional plane of a 
body (Barber and Brown, 1997). EIT is a non-invasive, inexpensive and portable imaging technique that can be used for lung monitoring applications.

However, EIT is relatively low resolution imaging technique that it is still in the research and development phase and has not yet been used clinically in the intensive care unit. It is important to validate EIT measurements and determine its accuracy to improve this imaging technology and minimize errors.

\subsection{Thesis Objectives:}

The objective of this thesis is to validate EIT regional lung measurements against simultaneous dynamic CT lung measurements. The implemented algorithm processes EIT and CT images of healthy and ALI diseased pig's lungs. It measures regional lungs behaviour and analyzes data obtained from both images to detect the percentage of accuracy. The thesis investigations include identifying and estimating five research parameters on the data obtained from both EIT and CT measurements, and finding the correlation between the estimated parameters on both images.

\subsection{Thesis Contributions:}

This thesis offers three novel contributions. First, an EIT validation technique developed to analyze regional measurements selected on EIT and CT images of chosen pigs' lungs through mechanical ventilation events. The implemented algorithm measures region of interests (ROI) on 
EIT images in comparison to regional measurements on simultaneous dynamic CT images. The implemented algorithm to process EIT and CT images is shown in figure 5-5 and figure 4- 7.

Second, this thesis processes and analyzes regional lungs measurements through the developed algorithm. ROIs were selected on different areas on the lungs for both healthy and ALI diseased pigs EIT and CT images. Data obtained from EIT and CT healthy pigs images were analyzed and compared to the same ALI diseased pigs images. Thesis shows that for some animals there are significant changes from healthy to ALI in $\tau_{1}$ and $\tau_{2}$ research parameters. It was found that these parameters poorly correlated between healthy and ALI for both EIT and CT images. EIT shows the changes in these parameters for more animals than CT. Thus, results suggest that EIT is a promising technology that can help to better understand ALI disease and give functional information about regional lungs ventilation.

Third, lungs physiological research parameters (explained in figure 4- 13) were developed and classified. The five research parameters were estimated on data obtained from EIT and CT images to detect the percentage of accuracy. It is found that $\mathrm{A} 1$ and $\mathrm{A} 2$ research parameters have negative correlation when comparing EIT to CT ROIs, whereas the other parameters are poorly correlated. The research parameters estimated on ROIs negatively correlate to ROIs y-coordinate locations on the EIT images and positively correlate to ROIs y-coordinate locations on the CT images. Thesis shows that EIT images have position errors when comparing EIT to CT ROIs. 


\section{Chapter 2}

\section{Background:}

This chapter presents background information related to the thesis. Breathing and lungs ventilation are discussed along with anatomy and physiology of the lungs. The background of EIT is presented in terms of its history, theory, and applications. CT technology is presented in terms of history, theory, and applications at the end of this chapter.

\subsection{Lung Ventilation:}

Humans need a constant supply of oxygen in order to stay alive and use it to break down food and to release energy and produce carbon dioxide as a waste product. This process happens continuously by taking oxygen from air, and expelling carbon dioxide (Moreno et al, 2007).

The respiratory system which is made up of organs such as the lungs, airways, and the respiratory muscles helps humans to breath. Lungs are one of the main organs of human respiratory system in which gas exchange occurs.

\subsubsection{Overview of Human Lung:}

Lungs are spongy organs lying in the thoracic cavity and in a group include lobes, lobules, bronchi, bronchioles, infundibula, and alveoli or air sacs (inner health group, 1996). Figure 2- 1 shows the anatomical components of human lungs and respiratory system. 


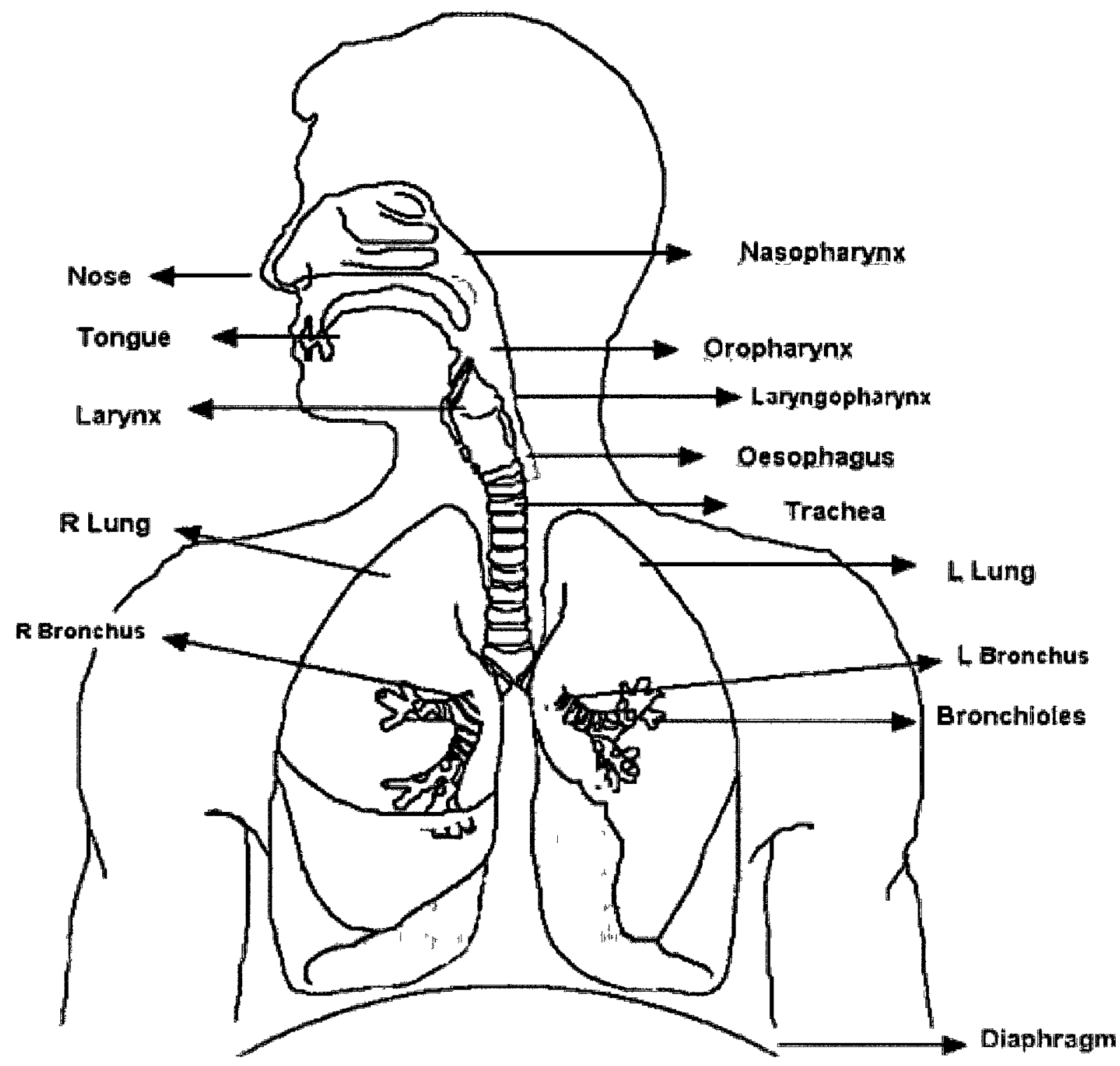

Figure 2- 1 : Basic parts of human respiratory system (Source: www.tutorvista.com/biology, 2010).

Air moves through the nose and mouth to get to the lungs. Inspired air gets heated to body temperature, and moisturized. When air enters the lungs, oxygen, gets absorbed into the blood and carbon dioxide, leaves it. 
Lungs contain about 300 million alveoli (Notter, 2000) which allows for generation of respiratory surface area up to $70 \mathrm{~m}^{2}$ in adult humans (Daniels and Orgeig, 2003). The exchange of respiratory gases between lungs and blood takes place by diffusion across layers of alveolar and capillary walls. Each alveolus is surrounded by small blood vessels called capillaries necessary for $\mathrm{O}_{2}$ and $\mathrm{CO}_{2}$, exchanges to take place (Nucleus Medical Art, 2005).

\subsubsection{Mechanism of Breathing:}

The process of breathing and exchanging gases, $\mathrm{O}_{2}$ and $\mathrm{CO}_{2}$, in the lungs is referred to as ventilation and pulmonary gas exchange. Figure 2- 2 shows the mechanic of inspiration or inhalation of fresh air into lungs and expiration or exhalation used air from lungs.
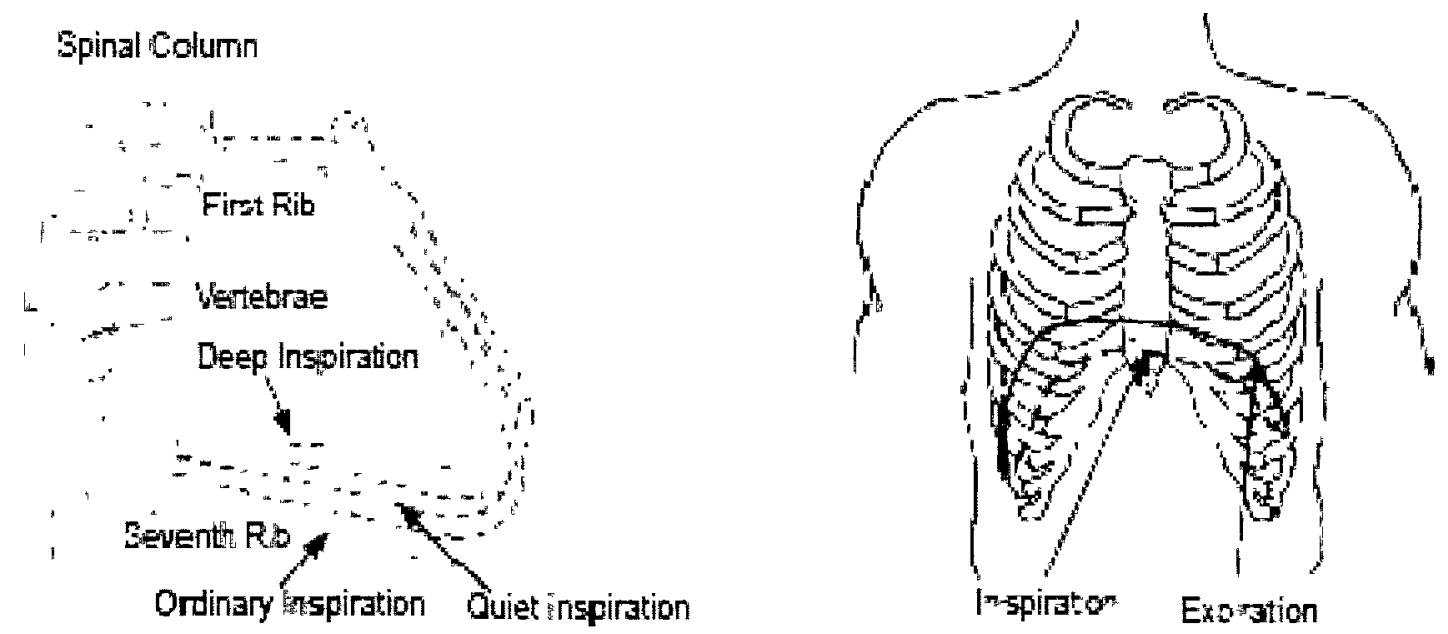

Figure 2- 2: Inspiration Process. Inspiration involves both raising the rib cage (left panel) and lowering the Diaphragm (right panel), both movements enlarge the volume of the thoracic cavity and draw air into the lung (Source: U. S. Navy Diving Manual, 2008). 
As seen in figure 2- 2, the volume of the lungs is increased by elevating the ribs and lowering the diaphragm. According to Boyle's law, the pressure should increase when the volume has decreased. Therefore, an increase in alveolar pressure occurs when the lung volume decreases, causing air to move from higher pressure regions to lower pressure regions, and this result in moving the air out of the respiratory system to environment (Chan et al, 1996).

During respiration, lungs expand and contract as the chest cavity changes size. The chest wall and diaphragm move down to enlarge the thorax cavity during inspiration and back up during the expiration (Chan et al, 1996).

In the process of respiration, air enters the lungs and then exits again through the trachea. With each complete breath, some air called residual volume stays in the lungs. Every time inspiration happens, additional air is added and, in the process of expiration, about the same volume that was inhaled gets removed.

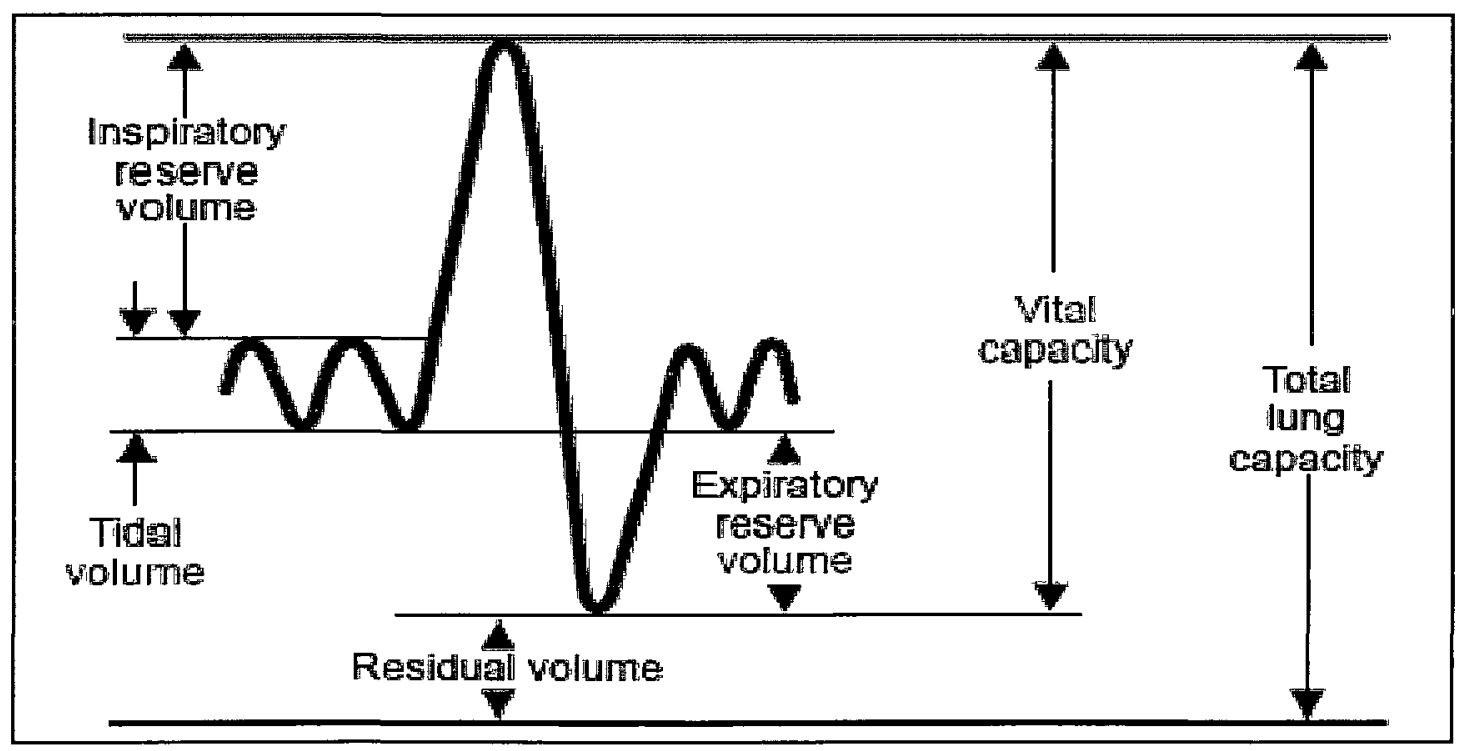

Figure 2- 3: Human lung volume (Source: U. S. Navy Diving Manual, 2008). 
As seen in figure 2- 3, the thick line is a subject breathing lung volume. The subject maximally inhales then maximally exhales after several normal tidal breaths. The volume of air moved during this maximal process is called the vital capacity. During exercise, breathing frequency and tidal volume increase. However, the tidal volume can never exceed the vital capacity. During a forced expiration, the amount of air remaining in the lungs is called the residual volume. The total lungs capacity is the sum of the residual volume and the vital capacity.

\subsubsection{Lungs Assessment:}

Lung volumes are different from person to another, depending on age, sex, body frame and fitness. Measuring total lung capacity is part of the systematic evaluation of lung function (Bokinsky, 1986).

Lung capacity could be affected by lung disorders such as asthma and emphysema. Measuring lung volumes can help detecting, characterising, quantifying the severity of lung diseases. Doing lung volume measurements is important to have accurate physiological diagnosis (Wanger et al, 2005).

\subsubsection{ALI/ARDS Lungs Disease:}

Acute Lung Injury (ALI) and Acute Respiratory Distress syndrome (ARDS) are syndromes of acute respiratory failure with different increasing severity of lung injury as a result of damage to cells and structures of the alveolar capillary membrane. It was first described by Ashbaugh and colleagues in 1976 (Nanchal et al, 2009). ALI/ARDS is a major cause of acute 
respiratory failure that develops in patients' lungs of all ages from a variety of clinical disorders such as sepsis (bacterial infection) and pneumonia (lungs alveoli filled with fluid) (Matthay et al, 2003).

The diagnosis of pulmonary infection in patients with ALI and ARDS is often difficult. The systematic signs of infection, such as fever, tachycardia, and leukocytosis are nonspecific findings in such patients (Fagon and Chastre, 2003).

It is defined as acute pulmonary inflammation as a result of increase in the pulmonary micro vascular permeability (Arora and Sankar, 1995). Also, it is categorized by sudden onset (less than 7 days) of acute hypoxemic respiratory failure and diffuses pulmonary infiltrates (Nanchal et al, 2009).

The main clinical diagnosis of ALI/ARDS associate with the following features (Calffee, 2009).

the acute arrival of Bilateral diffuse pulmonary infiltrates on chest radiograph $\mathrm{PaO}_{2}$, partial pressure of oxygen in arterial blood, to $\mathrm{FiO}_{2}$, fraction of inspired oxygen, ratio of less than 300 (less than 200 defines ARDS) 


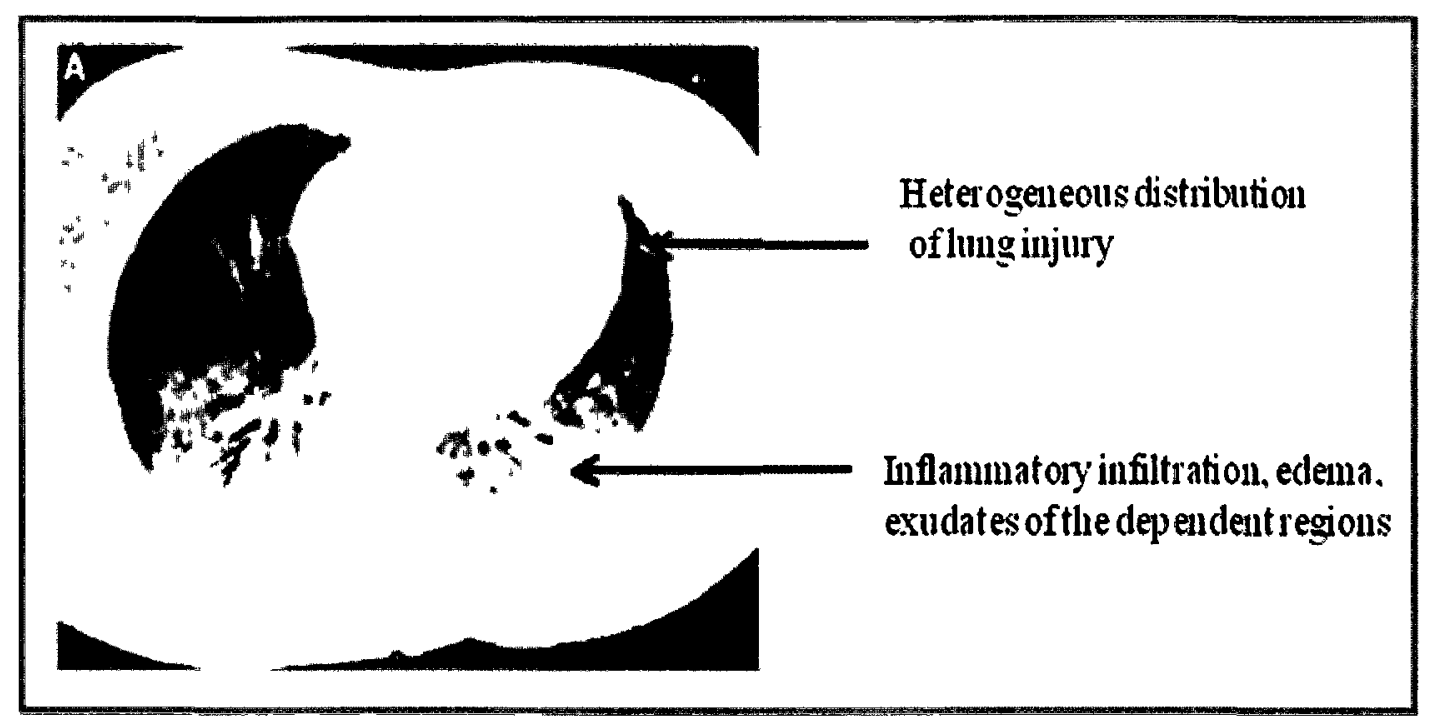

Figure 2- 4: CT image of patient with ARDS showing heterogeneous distribution of lung injury and the inflammation infiltration (Source: Rotta and Steinhorn, 2007).

As seen in figure 2- 6, ARDS patient CT image of the lungs showing the inhomogeneous distribution of lungs injury and inflammatory infiltration $\mathrm{t}$ hat $\mathrm{m}$ ake $\mathrm{l}$ ung $\mathrm{v}$ entilation so challenging (Rotta and Steinhorn, 2007).

ALI/ARDS is still under-diagnosed and undertreated. Meanwhile, research activities are ongoing attempting to discover more biological indications for this disease (Calfee, 2009).

\subsection{Electrical Impedance Tomography:}

\subsubsection{EIT History:}

Electrical Impedance Tomography (EIT) is a method developed in the early 1980s by Barber and Brown to produce cross s ectional images of a $\mathrm{n}$ o bject $\mathrm{f}$ rom $\mathrm{m}$ easurements of electrical impedance at its boundaries (Frerichs et al, 1999). EIT calculates a cross s ectional 
image of a body by placing a series of electrodes around it and recording electrical measurements taken at the boundaries to calculate the change in conductivity distribution of different tissues in the body (Adler et al, 1998).

\subsubsection{EIT Theory:}

According to Ohm's law, when a known current passes through an object and the voltage across it is known, then the resistance of the object can be calculated. The principle of EIT uses Ohm's law that is a number of impedance measurements are made from electrodes placed on the boundary of an object (Holder, 1992).

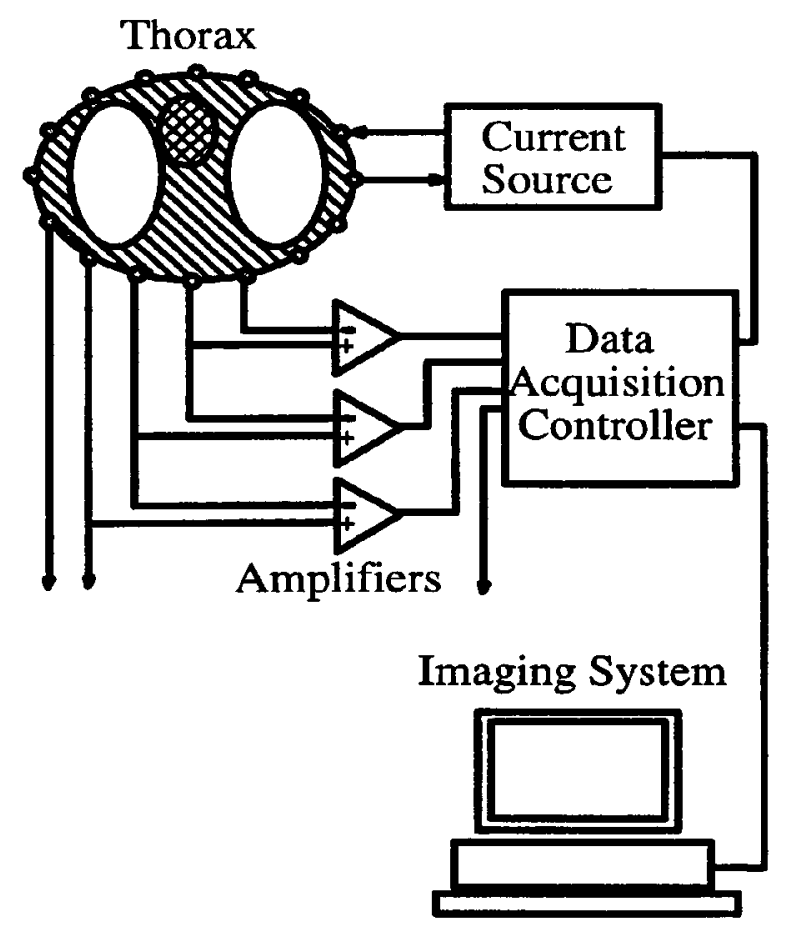

Figure 2- 5: A block diagram of EIT system (source: Adler et al, 1998). 
As seen in Figure 2-5, an EIT system block diagram showing 16 electrodes attached (equally spaced) on the circumference of a thorax. A current of $50-\mathrm{mA}$ at $50-\mathrm{kHz}$ is applied across each adjacent pair of electrodes while the voltage distribution is measured between each set of neighbouring electrodes. The process is repeated for each pair of electrodes and measurement of distributed voltages is recorded until a set of EIT data is completed and sent to a computer where EIT images of change in conductivity distribution is calculated (Adler $e t$ al, 1998).

For most biological measurements, an alternating current at high frequency (e.g., above $10-\mathrm{kHz}$ ) is used to avoid risk of exciting nerves (Holder, 1992). Applying an alternating current at a different frequency may affect the behaviour of the body. For example, a current applied (e.g., above $10-\mathrm{mA}$ ) at frequency $60-\mathrm{Hz}$ makes the chest muscles contract and it becomes harder to breathe (Geddes and Roeder, 2006).

Different biological tissues have different electrical properties (Frerichs, 1999), conductivity and permittivity, related to the tissue dimensions, internal structure, and arrangement of the constituent cells. Body tissues electrical resistivity is in the range of $0.65 \Omega \mathrm{m}$ for cerebrospinal fluid to $150 \Omega \mathrm{m}$ for bone (Pandya et al, 2009). For example, EIT can determine changes in lung tissue electrical properties which get modified by local variations in gas volume (Frerichs et al, 2001).

Estimating the inner tissue electrical impedance distribution and calculating the mathematical values for current and potential from conducting surface is an inverse problem (Pandya et al, 2009). Therefore, the measured voltages on the attached electrodes at the objects boundaries are needed for the process of EIT image reconstruction (Movafeghi et al, 2004). The 
following equation (2.1) shows the relationship $b$ etween $t$ he $b$ oundaries $m$ easurements a nd conductance distribution inside the object.

$$
v=A_{c} c
$$

Where $\boldsymbol{v}$ is a vector of all voltage values, $\boldsymbol{c}$ is a vector of conductivity values, and $\boldsymbol{A}_{\boldsymbol{c}}$ is a matrix representing the transformation of $\mathrm{t}$ he $\mathrm{c}$ onductivity vector i nto $\mathrm{t}$ he $\mathrm{v}$ oltage $\mathrm{v}$ ector (Barber, 2000).

EIT images can be reconstructed in different ways such as absolute, time difference, and frequency difference. Images reconstruction methods are very sensitive to errors may result from incorrect measurements (Hartinger et al, 2007), due to electrode movement or body sweat, and lead to image artefacts and distortion.

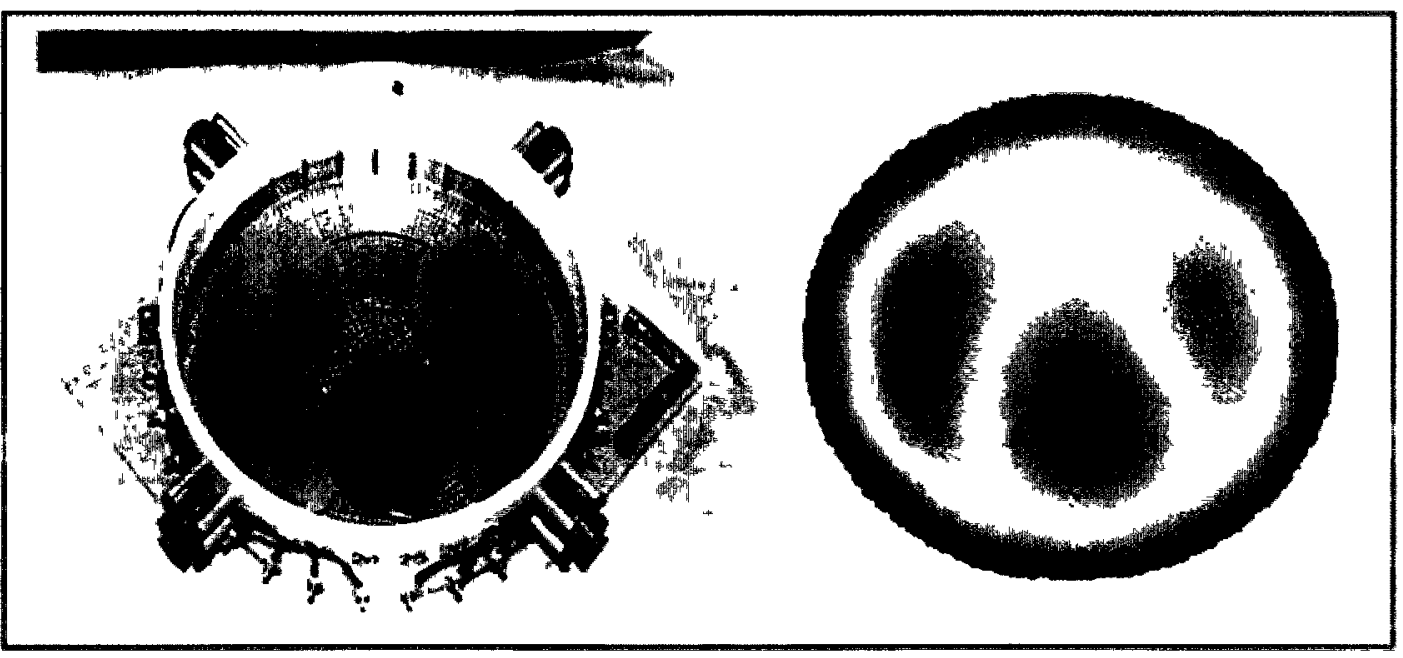

Figure 2- 6: (left) thorax phantom with pink agar lungs, blue agar heart and black skin in saline. The resistivity of heart is $150 \mathrm{ohm}-\mathrm{cm}$, lungs are $1000 \mathrm{ohm}-\mathrm{cm}$, and background saline is $300 \mathrm{ohm}-\mathrm{cm}$. Figure (right), an EIT colour sensitivity image reconstructed of the of the thorax phantom (source: Rensselaer Polytechnic Institute, Electrical Impedance Imaging Laboratory, www.ecse.rpi.edu, 2010). 
To assess and compare the performance of EIT systems under test and studies, phantoms developed to produce EIT measurement sets or images of the phantom. Figure 2- 5 (left) shows a sample phantom thorax with 32 stainless steel electrodes. The phantom has two lung-shaped structures and heart-shaped structure. The right figure shows colour sensitivity EIT image reconstructed from data obtained from this test phantom (Rensselaer Polytechnic Institute, 2006).

\subsubsection{EIT Applications:}

EIT has promising different applications in medicine, geophysics, environmental sciences and non-destructive testing of materials. For example, EIT can be used in medical applications to detect pulmonary emboli, monitoring of apnoea, monitoring of heart functions and blood flow, and breast cancer detection (Borcea, 2002). EIT can be used as a technique to measure lung volumes and gastric motility by imaging the conductivity changes in the lungs and abdomen (Adler et al, 1994, 1998). It can provide a continuous monitoring for neonate lung function to reduce the risks of chronic lung disease in infancy (CLDI) in intensive care units (ICU) (Bayford et al, 2007).

EIT is still in research and development phase and has not been fully implemented such as in the intensive care unit yet. However, EIT technique is non-invasive, non-destructive, portable, promises high temporal resolution, continuous monitoring, relatively low cost, and the possibility that soft tissue changes may be imaged which are invisible to other imaging techniques (Lionheart et al, 1998).

EIT can help optimize treatment and help monitor lungs ventilations. It can be used for better assessment and discover ALI/ARDS diseases in early stages. Functional EIT technique is a 
new evaluation approach to image the lung function and monitor the development of experiment lung injury (Frerichs et al, 1998).

Lung regions differ in their mechanical behaviour. Therefore, regional EIT imaging can be helpful to better assess patients with ALI because it separates different regional ventilation behaviour of the lungs (Putensen et al, 2006).

\subsection{Computed Tomography:}

Background information about CT technology is presented in this chapter because EIT images are validated in this thesis project using simultaneous dynamic CT images. Data from both images are processed and analyzed.

\subsubsection{CT History:}

Computed Tomography (CT) is a non-invasive radiographic technique developed for, and primarily used as a diagnostic tool in the field of medicine (Applbaum, 2005). Since the foundation of CT in 1970s, its use has increased rapidly recording more than 62 million CT scans per year in the United States (Brenner and Hall, 2007). CT examination can be used to help detect a wide range of abnormalities resulting from injuries or illness in any part of a body. For example, it can be used to help diagnose or monitor treatment for cancer, infections, inflammatory disorders, causes of abdominal pain, and results from injury such as head bleeding from car accidents (Radiological Society of North America, 2010). 


\subsubsection{CT Theory:}

CT imaging technique work on the basis of $\mathrm{x}$-ray examinations. It uses an $\mathrm{x}$-ray tube and a series of electronic x-ray detectors rotate around an object (Applbaum, 2005). During a complete CT machine rotation, fan-shaped x-ray beams are emitted from different angles x-ray tubes. Some x-ray photons get absorbed by the body and some get passed through the patient's body to be recorded at the $\mathrm{x}$-ray detectors. The amount of the absorbed $\mathrm{x}$-ray from each subject or organ being examined differs depending on subject size and atomic numbers of atoms in the subject and the concentrations of molecules that comprises the subject. Iodine and barium have high atomic numbers and therefore increase $\mathrm{x}$-ray absorption when using contrast media (Hayashi et al, 2010). However, when using contrast material with ALI diseased patients, the injection of contrast material induces an apparent increase in lung tissue but may worsen the lung injury because of contrast material leakage into the lung parenchyma (Bouhemad et al, 2002). 


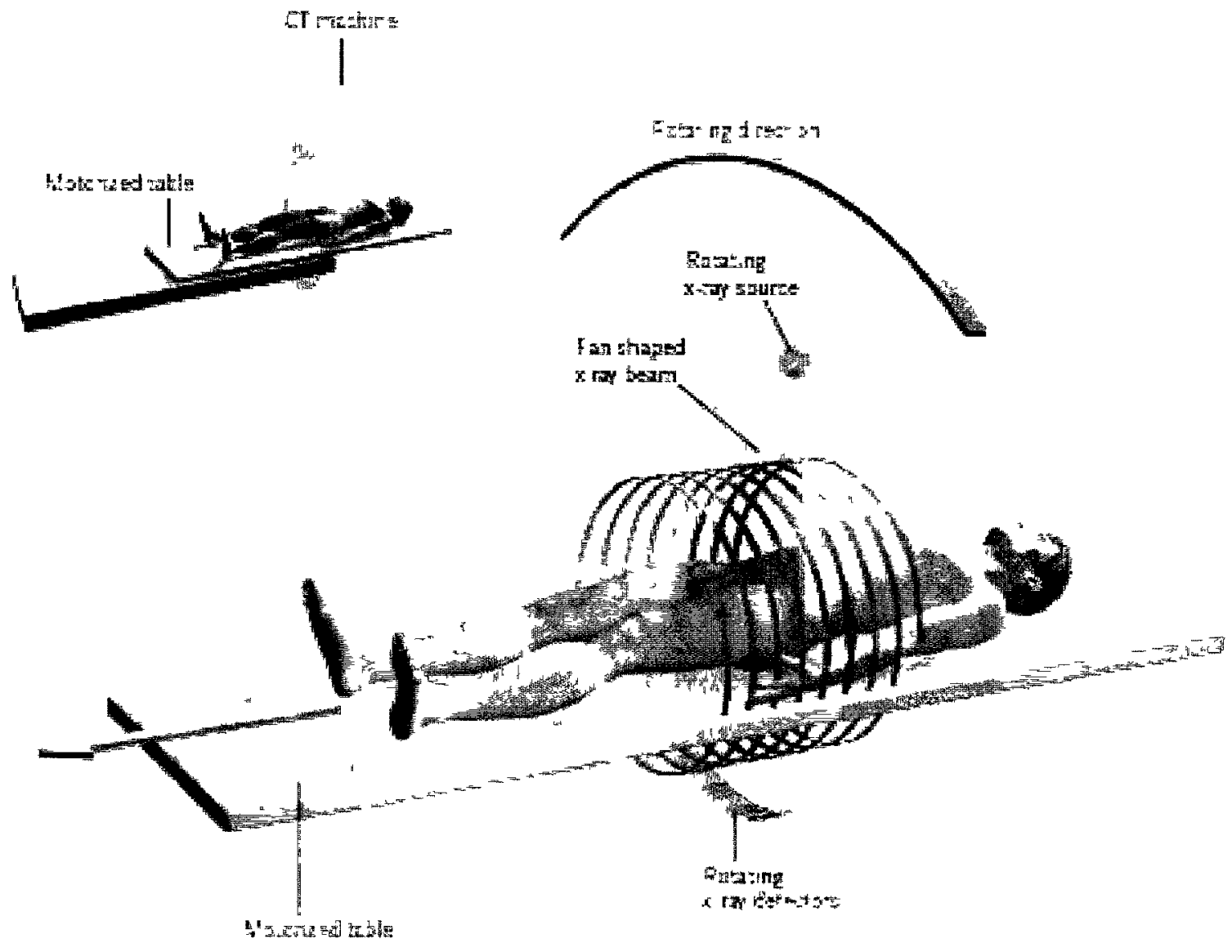

Figure 2- 7: CT technique, showing the main components used in the CT machine, X-ray source, beam, and detectors are shown in position with respect to human body on the motorized table (source: Brenner and Hall, 2007).

\section{As seen in figure 27, CT machine has motorized Gable that moves the pathent throngh}

The x-ray source rotates within the circular opening and at the same time, the x-ray detectors rotate synchronously on the other side of the patient. The x-ray source produces a narrow fan shaped beam (width range, 1-20 mm). The figure shows only single row of $\mathrm{x}$-ray detectors. However, current CT machines typically have multiple rows (at different angle) of detectors operating side by side to simultaneously image many slices to reduce the overall scanning time. X-ray detectors from each angle record the x-rays beams passing through the whole body. 
A cross section image of body tissues and organ is formed from the collected data from different angle around the body (Applbaum, 2005). All data acquired are processed by computer to produce a series of image slices representing body targeted region (Brenner and Hall, 2007). Data acquired can be manipulated to form images in different planes to have 2 and 3-D images (Applbaum, 2005).

There are different $\mathrm{CT}$ image reconstruction techniques such as iterative reconstruction (IR) and conventional filtered back-projection (FBP). IR is an algorithm which computes the final image through many loops of forward and backward projection. While FBP reconstructs the image by filtering the projection data and performs a back-projection once to the filtered data ( $\mathrm{Li}$ et al, 2005).

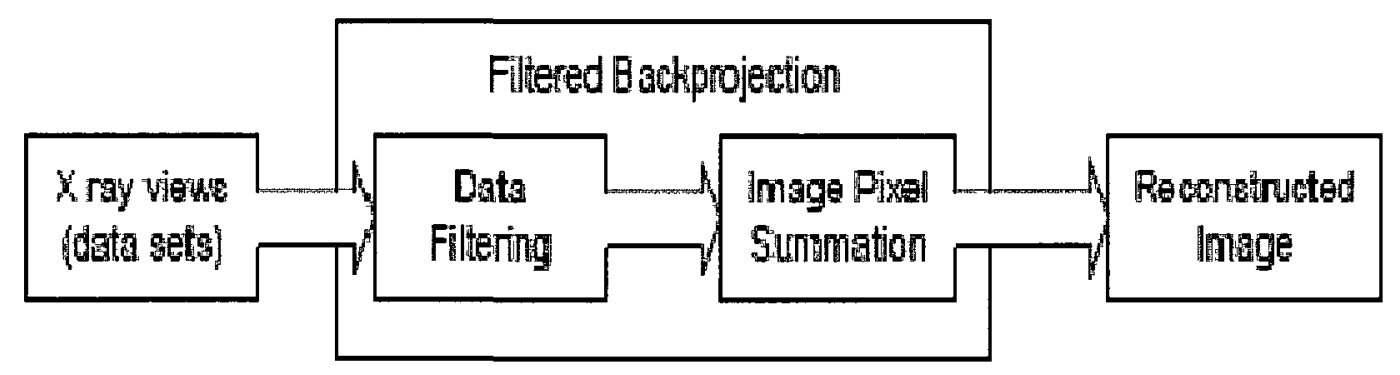

Figure 2- 8: CT scan image reconstruction (Source: Pointer, 2008).

Figure 2- 8 shows FBP technique in which hundreds of $x$-ray image data sets obtained by the CT scanner is filtered as preparation for the back-projection step which is adding each filtered $x$-ray image data set's contribution into each pixel of the final image reconstruction (Pointer, 2008). The following figure 2- 9 shows an example of a CT cross sectional image of the chest showing the lungs, heart, and thorax. 


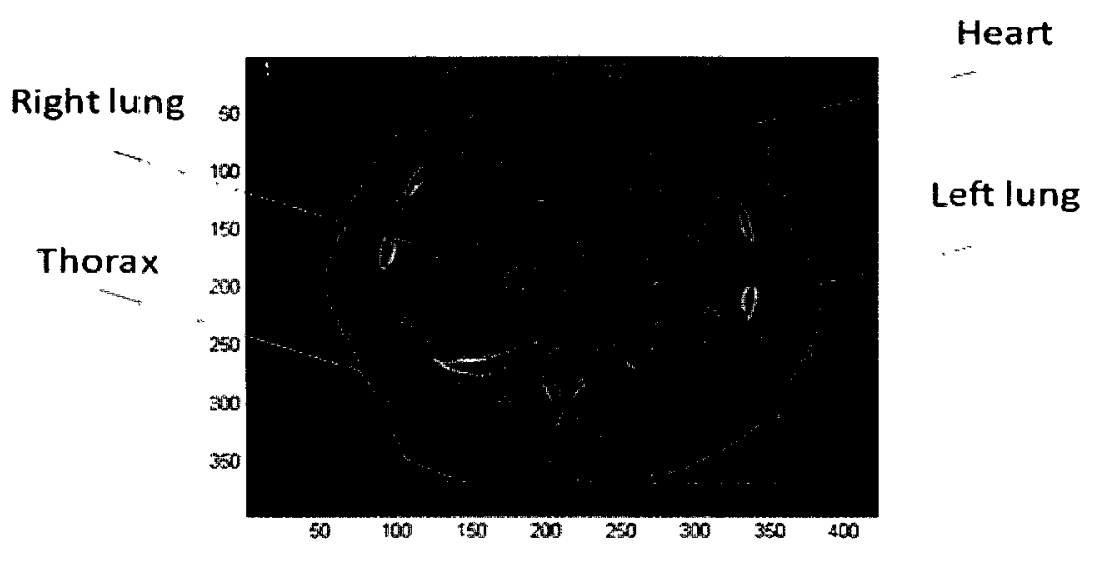

Figure 2- 9: CT cross sectional image of a pig chest showing the lungs left and right and the heart.

\subsubsection{CT Applications}

CT provides very detailed image of a body's organs such as bones, soft tissues, or blood vessels. It is painless, non-invasive, a nd a ccurate i maging $t$ echnique $u$ sed $f$ or diagnosis, treatment, and management of diseases (Brenner and Hall, 2007). It is important and sometimes life saving tools for diagnostic medical e xaminations a nd $g$ uidance o f $\mathrm{i}$ nterventional a nd therapeutic procedures. Also, CT allows rapid acquisition of high resolution three-dimensional images, providing radiologists and other physicians with cross sectional views of patient's body anatomy (Health Care Human Factors Group, 2006).

With the details CT image provide, it $\mathrm{h}$ as been r egularly u sed in intensive care $\mathrm{u}$ nit. However, $x$-ray ionizing radiation resulting from $\mathrm{C} T \mathrm{~s}$ can $\mathrm{m}$ achine $\mathrm{c}$ an a ffect o $\mathrm{n}$ the D NA directly causing body cells damage (Brenner and $\mathrm{Hall}, 2007$ ). A lso, there is a risk of serious allergic reaction to contrast agent that contains iodine which increases organ $\mathrm{x}$-ray absorption (Radiological Society of North America, 2010). 


\section{Chapter 3}

\section{Data Collection:}

EIT is an imaging technique that is still in research and development stage. It has not been clinically used in intensive care unit yet, but it has promising applications. In medicine, it can be used to measure regional changes in lung volume (Frerichs et al, 2001). EIT can be used to assess ALI/ARDS patients and discover the diseases at early stages. EIT can be applied to monitor the development of experiment lung injury (Frerichs et al, 1998).

The goal of the research is to validate EIT regional lung measurements against simultaneous dynamic CT lung measurements and to investigate the percentage of accuracy and errors in EIT measurements when comparing to CT measurements. Therefore, EIT and CT data were collected from research experiment done on life pigs.

EIT and CT data were collected at Johannes Gutenberg University in Mainz, Germany, by team lead by Dr. Marc Bodenstein before the research is conducted. The research experiment was done in the Animal laboratory, "Verfügungsgebäude".

This chapter explains the basic characteristics of studied pigs, the devices and tools utilized while collecting the data, and the procedure used to collect both data. The ventilation applied to the pigs along with the data collected are explained as well. 


\subsection{Characteristics of the Studied Pigs:}

Eleven pigs, Anaesthetized and paralysed, were considered for this research study. The ethics committee at Johannes Gutenberg University approved the research project and the protocols for all procedures applied in this study. The experiment protocol is based on the work of New England Journal of Medicine (NEJM) described by Gattinoni et al, 2006.

The pigs were studied for the same amount of time at a different date within a period of almost one month. "Warmed Sterofundin" is a lung lavage model that was used in the research study. It is a model of ALI/ARDS diseases applied to healthy pigs. A fluid gets pushed into the lungs and then removed out of the lungs causing experiment lung injuries. Data were collected from each pig before and after applying ALI/ARDS lung lavage model.

At the end of this research study, each animal life was terminated by giving an overdose of anesthetic, "Euthanasia Propofol Potassium". Basic characteristics of the studied 11 pigs are shown in table 3-1.

\begin{tabular}{|c|c|c|c|c|}
\hline $\begin{array}{c}\text { Pig Number } \\
\text { (ards22-) }\end{array}$ & Date & Weight/Kg & $\begin{array}{c}\text { Number of } \\
\text { lavage }\end{array}$ & $\begin{array}{c}\text { Early } \\
\text { Death }\end{array}$ \\
\hline 01 & $22 / 03 / 2007$ & 22 & 2 & Yes \\
\hline 02 & $23 / 03 / 2007$ & 22 & 2 & No \\
\hline 03 & $26 / 03 / 2007$ & 22 & 1 & No \\
\hline 04 & $28 / 03 / 2007$ & 23 & 3 & No \\
\hline 05 & $29 / 03 / 2007$ & 24 & 3 & No \\
\hline 06 & $30 / 03 / 2007$ & 23 & 2 & No \\
\hline 07 & $12 / 04 / 2007$ & 22 & 1 & No \\
\hline 08 & $16 / 04 / 2007$ & 18 & 3 & No \\
\hline 09 & $19 / 04 / 2007$ & 25 & 0 & Yes \\
\hline 10 & $26 / 04 / 2007$ & 22 & 1 & No \\
\hline 11 & $27 / 04 / 2007$ & 23 & 3 & No \\
\hline
\end{tabular}

Table 3- 1: characteristics of the 11 studieghpigs used for the research experiment 
As seen in table $3-1$, the studied pigs were labeled and numbered from 01 to 11 . The research experiments were conducted on each pig on a different date. The weight for the pigs was in the range 18 to $25 \mathrm{~kg}$. Each pig had lungs lavage for $0,1,2$, or 3 times. Some pigs died in the process of conducting the research experiment (pig number 01 and 09).

\subsection{Devices and Tools Used:}

The CT scanner used in the research study is "Siemens Somatom plus 4"; it has $2 \mathrm{x}$-ray tube sources and 2 detectors. It was set to acquire dynamic CT images at frequency rate of $10 \mathrm{~Hz}$. The image reconstruction time was 1.5 images per second.

The EIT measurements were acquired using Göttingen EIT GoeMF II system (Carefusion, Hochberg, Germany). The system acquires data at frequency rate 13 frames per second. EIT measurements were taken at $50-\mathrm{mA}$ current and $50-\mathrm{KHz}$ frequency through the EIT system.

Sixteen $\mathrm{Ag} / \mathrm{AgCl}$ electrodes were used for taking the EIT measurements. The electrodes were wired connected to the EIT system. 


\subsection{Data Collection Procedure:}

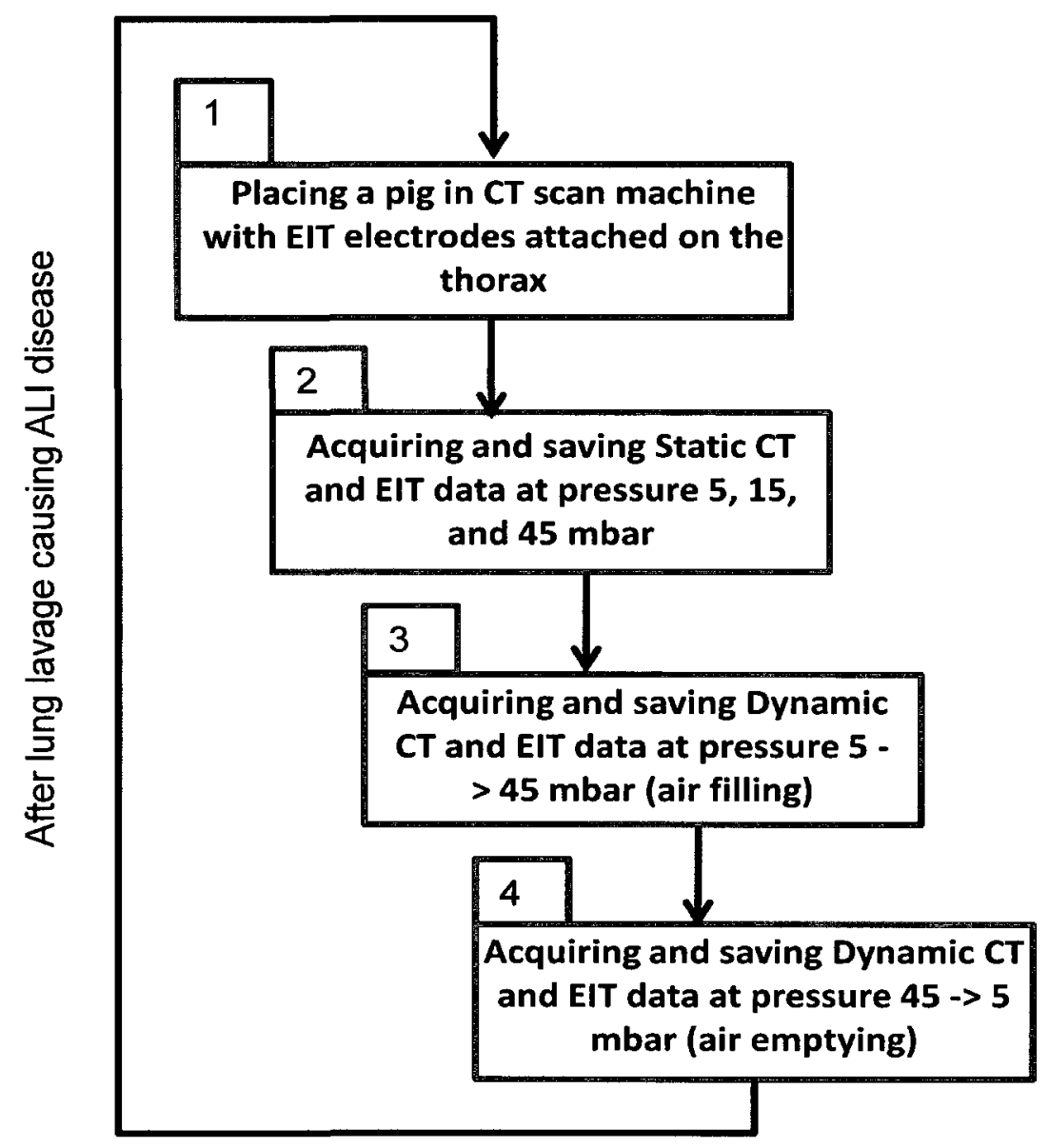

Figure 3-1: Diagram showing data collection procedures.

As shown in figure 3-1, EIT images and dynamic and static CT images were acquired in

4 steps in which data were saved to a computer. The following explain each step:

1. Each studied pig was placed in a CT scanner with 16 EIT electrodes were attached, equally spaced, to its chest circumference. 
2. Static CT and EIT images were acquired at air pressure 5, 15, and then 45 mbar and saved to a computer.

3. Dynamic CT and EIT images were acquired at air pressure $5 \rightarrow 45$ mbar (air filling) and saved to a computer.

4. After 30 seconds, Dynamic CT and EIT images were acquired at air pressure $45 \rightarrow$ 5 mbar (air emptying) and saved to a computer.

The process ( 1 to 4 ) was repeated once after inducing lung lavage to the same pig causing ALI disease.

\subsection{Lung Ventilation Applied:}

Each pig was assigned to receive pressure-controlled mechanical ventilation. Continuous positive airway pressure (CPAP) technique was used to obtain static and dynamic CT.

$>$ Static ventilation, the lungs get inflated at different levels with a constant air pressure 5, 15, and 45 mbar continuously. Static CT images visualize lungs at end-expiration and end-inspiration. It is 3D images of different cross sections of the lungs

Dynamic ventilation, the lungs get inflated with air pressure from 5 to $45 \mathrm{mbar}$, air filling, for a period of 30 seconds. Then, it gets deflated back to normal with air pressure 45 to 5 mbar, air emptying. Dynamic CT images visualize the lungs by capturing regional effects of the lungs during complete respiratory cycle in 
mechanical ventilation (David et al, 2005). It is 2D images of the same cross section of the lung taken through sequence time.

Pigs were ventilated before and after lung lavage. EIT and CT data were recorded for healthy and ALI/ARDS diseased pig. For the CT data obtained, events applied on each pig were determined according to the starting and end time.

For the EIT data obtained, the events were recognized by the EIT file name. For example, pig labeled "ards22-02", the air filling started at time equal 14:49, and ended at time equal 15:09. The EIT data for this event were saved under name "unimzards2202031", where "unimzards22" is a general name for all EIT files, "-02" indicates the pig's number out of the eleven pigs, and " 031 " is a sequenced number for each EIT file saved.

For each pig measurements, CT and EIT data were collected and saved in the computer. Also, the details regarding each pig were recorded on an excel sheet showing the pig name, events applied, the time of the events, and the EIT file name. All CT and EIT data obtained from all studied pigs were saved under folders, each named by the date of the measurements. 


\section{Chapter 4}

\section{CT Analysis:}

This chapter describes the methodology developed to analyze the dynamic CT image data. It analyzes dynamic CT images obtained from healthy and ALI diseased selected pig through the ventilation air filling and then air emptying. The section contains a block diagram showing the algorithm implemented to analyze the CT data. Images and graphs describing the $\mathrm{CT}$ analysis algorithm are shown as well.

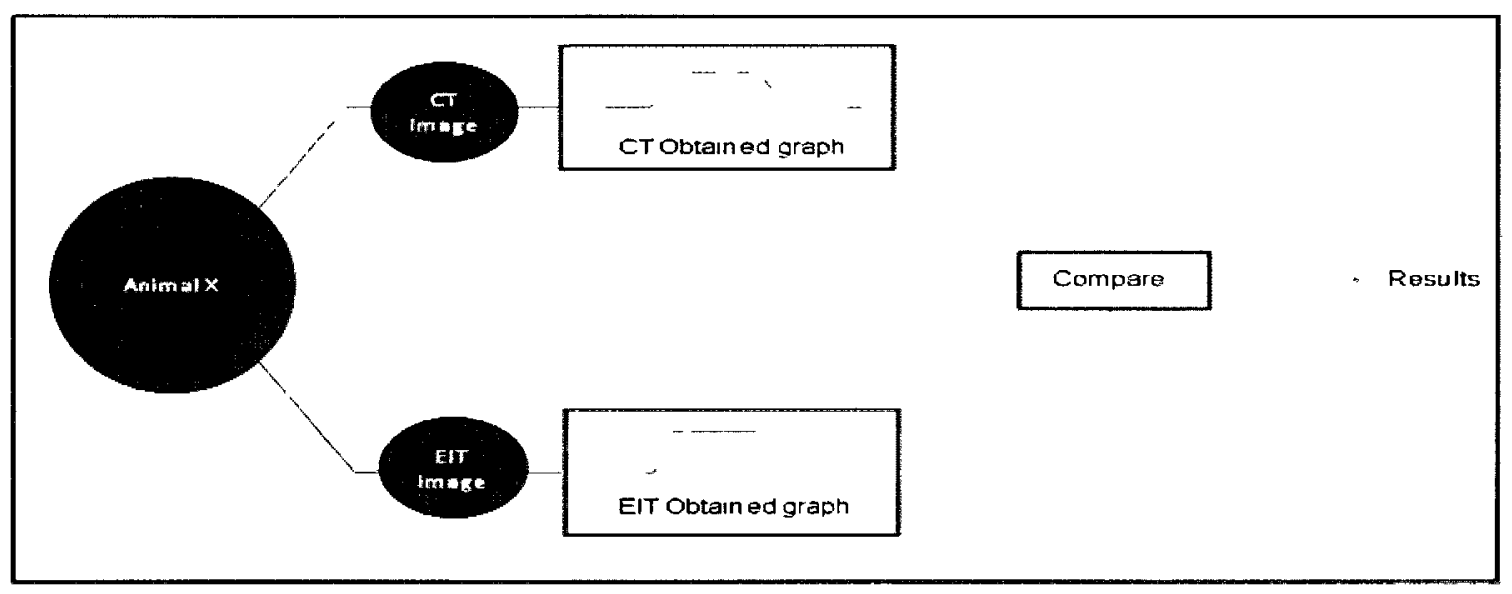

Figure 4- 1: Research project overview, EIT and CT images of the lungs were taken from a selected pig x. EIT and CT Graphs data are processed and compared to each other.

As seen in figure 4-1, a research project overview showing the main steps of this thesis project. EIT and CT images of the lungs were obtained from a selected pig. MATLAB is used to implement and develop an algorithm to analyze both EIT and CT data. Research results are compared and discussed in details at the end of this research. 


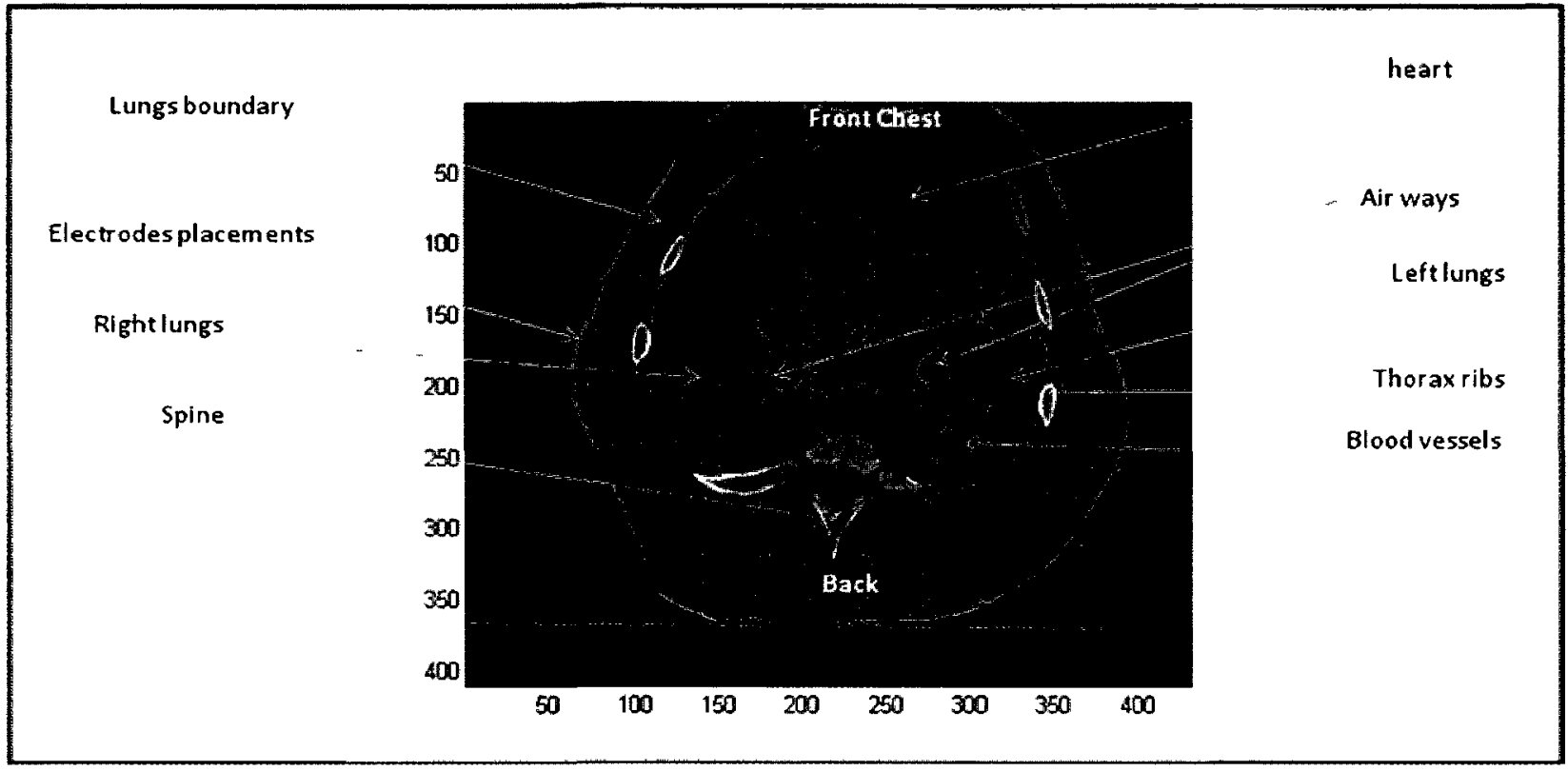

Figure 4- 2: CT image of a chest cross section of a health pig labelled "ards22-03" (no lung injury), showing the lungs, heart and the main other components in the image.

As seen in figure 4-2, cross sectional CT image of a healthy pig's chest showing the lungs, the heart, air and blood vessels, spine, and thorax ribs. The top part on the image shows the chest, the bottom part shows the back of the pig. Also, the boundaries of the lungs and the placements of the electrodes are shown in the image. 


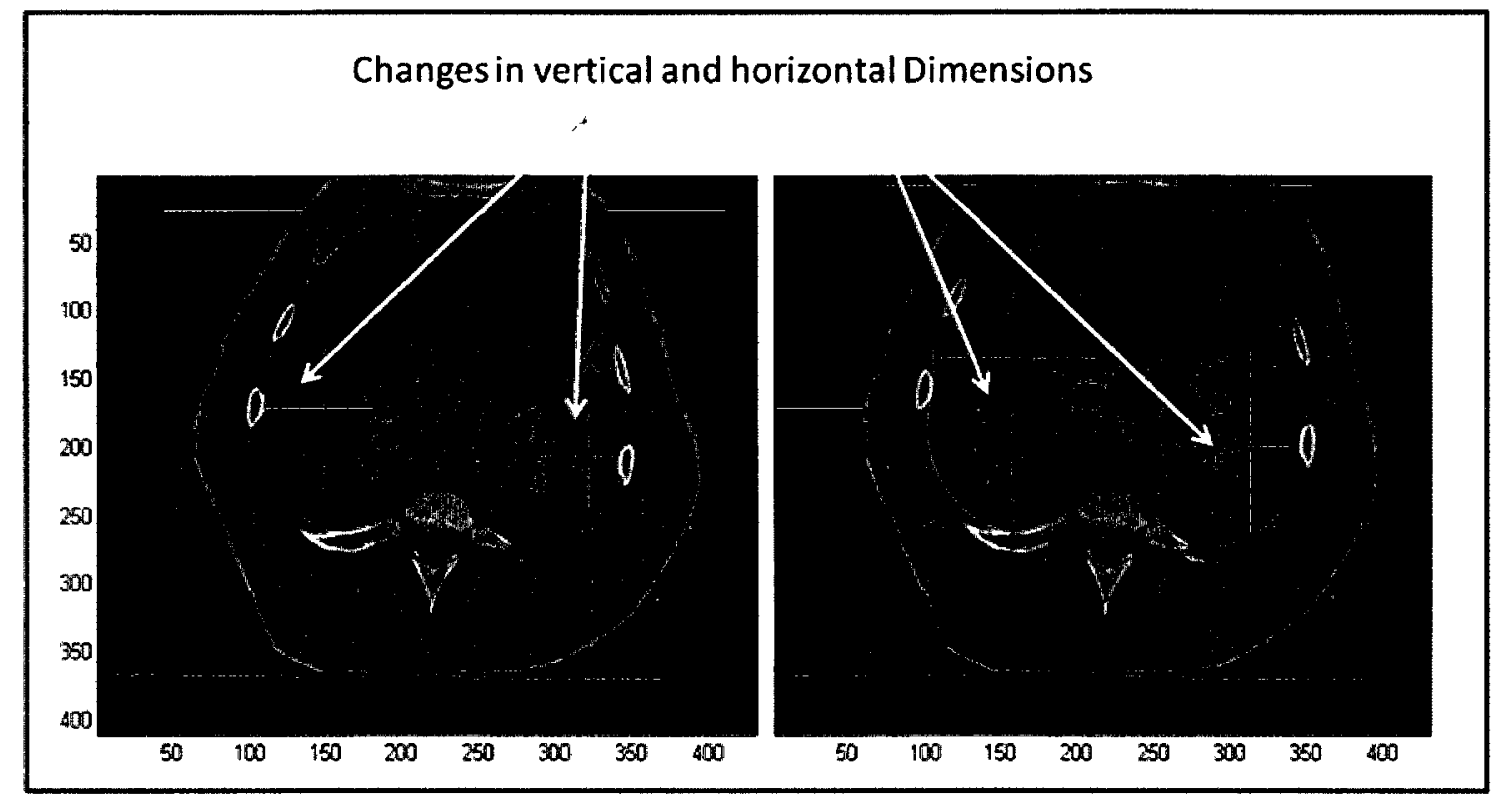

Figure 4- 3: CT images of a chest cross section of a health pig labelled 'ards22-03' (no lung injury), (left) lungs before air filling $(\mathrm{Paw}=0 \mathrm{cmH} 2 \mathrm{O}$ ), (right) lungs after air filling $(\mathrm{Paw}=40 \mathrm{cmH} 2 \mathrm{O})$. Lines indicate change in size. Note the change in lung size and chest vertical and horizontal dimensions.

As seen in figure 4-3 (left), CT image of cross section of the lungs of a sample healthy pig before applying air filling. It shows the size of the boundaries and the dimensions of the lungs.

Figure 4-3 (right) shows a cross section CT image of the lungs for the same healthy pig's lungs after applying air filling. The lungs and boundaries expanded and changed in shape and size; the vertical and horizontal dimensions of the lungs increased. 


\section{Changes in vertical and horizontal Dimensions}
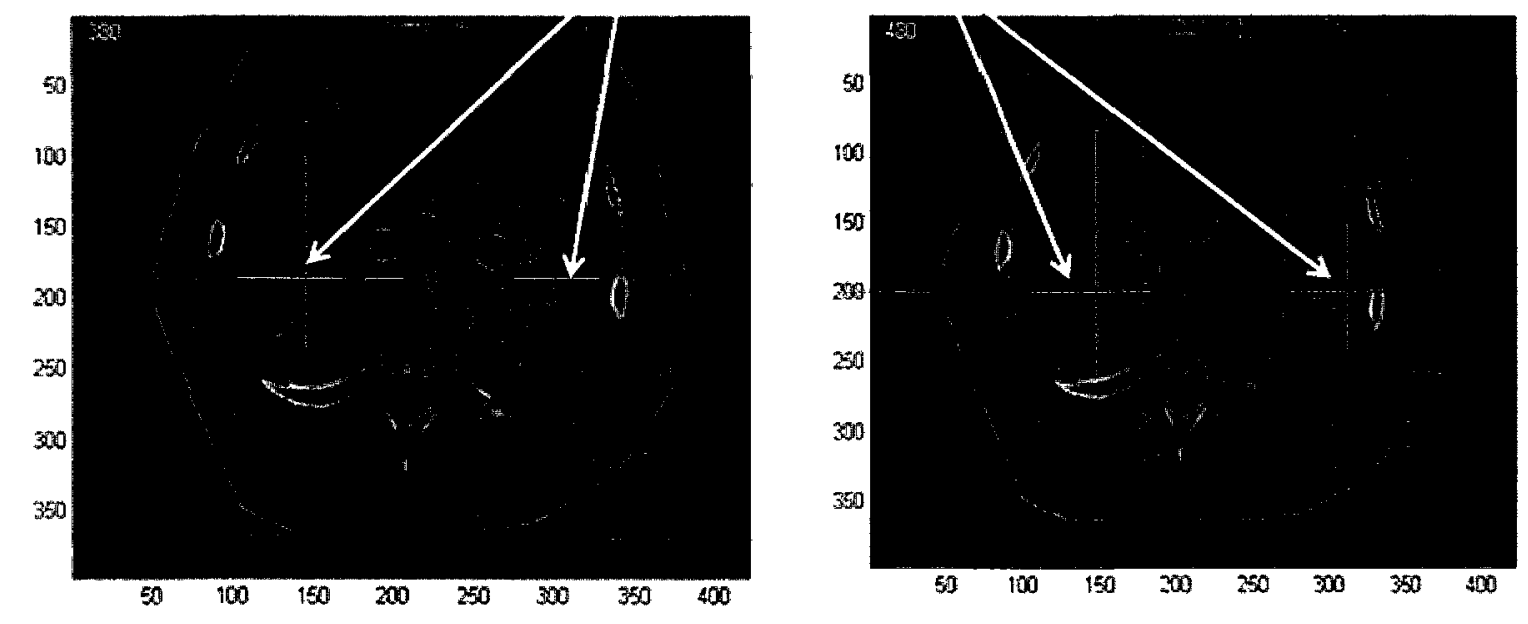

Figure 4- 4: CT images of a chest cross section of a health pig labelled "ards22-03" (no lung injury), (left) air filled lungs $(\mathrm{Paw}=40 \mathrm{cmH} 2 \mathrm{O})$, (right) air emptied lungs $(\mathrm{Paw}=0 \mathrm{cmH} 2 \mathrm{O})$. Lines indicate change in size before and after air emptying. Note the change in lung size and chest vertical and horizontal dimensions.

Figure 4-4 (left) shows CT image of cross section of the lungs of a sample healthy pig before the air emptying. The image on the right shows a cross section of the lungs for the same pig after air emptying in which the lungs contracted and decreased in vertical and horizontal dimensions.

When applying lungs lavage to the same pig, the lungs get injured and accumulate fluid at the bottom. The same ventilation events were applied to the pig after lungs lavage. Dynamic CT images were obtained through the air filling and air emptying. ALI diseased pig data were analyzed and studied. 


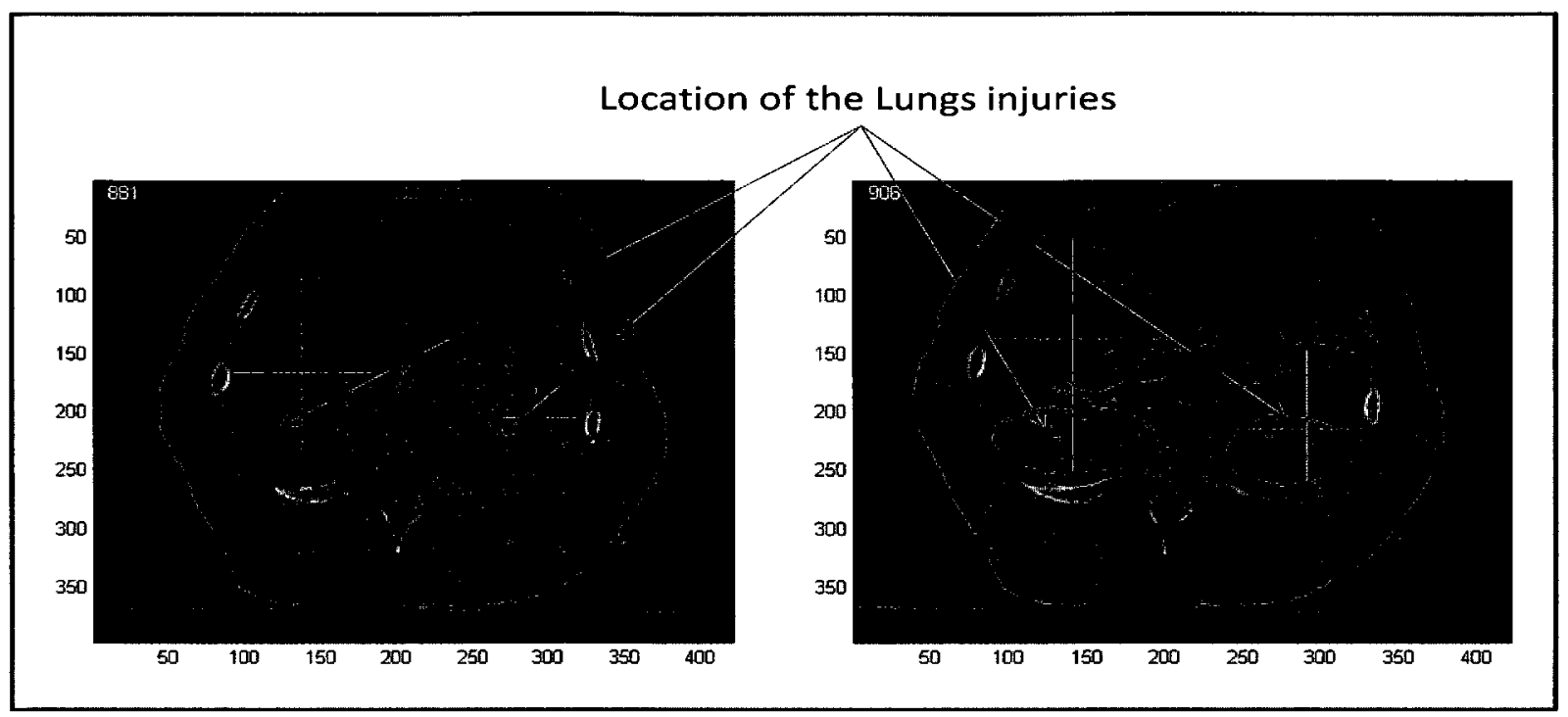

Figure 4- 5: CT images of a chest cross section of ALI diseased pig labelled "ards22-03", (left) image of the lungs before air filling ( $\mathrm{Paw}=0 \mathrm{cmH} 2 \mathrm{O}$ ), (right) the lungs after air filling ( $\mathrm{Paw}=40 \mathrm{cmH} 2 \mathrm{O}$ ). Note the change in lung size and chest vertical dimension as well as the change in shape of the heart and the movement of air and blood vessels. The circled areas show the result of injured lungs, before and after air filling.

As seen in figure 4-5, the left image shows a cross sectional CT image of ALI diseased pig's lungs before air filling. It shows the lungs injuries (blue circles) at the bottom of the image as a result of ALI disease.

The right image in figure 4-5 shows the same pig lungs after the air filling. The lungs get inflated and change in shape and size (increased vertical and horizontal dimensions of the lungs). The lung boundaries became bigger and their heart shape and location change as a result of lung expansion. The blue circles indicate the location of the lungs injuries after the lungs get inflated. 


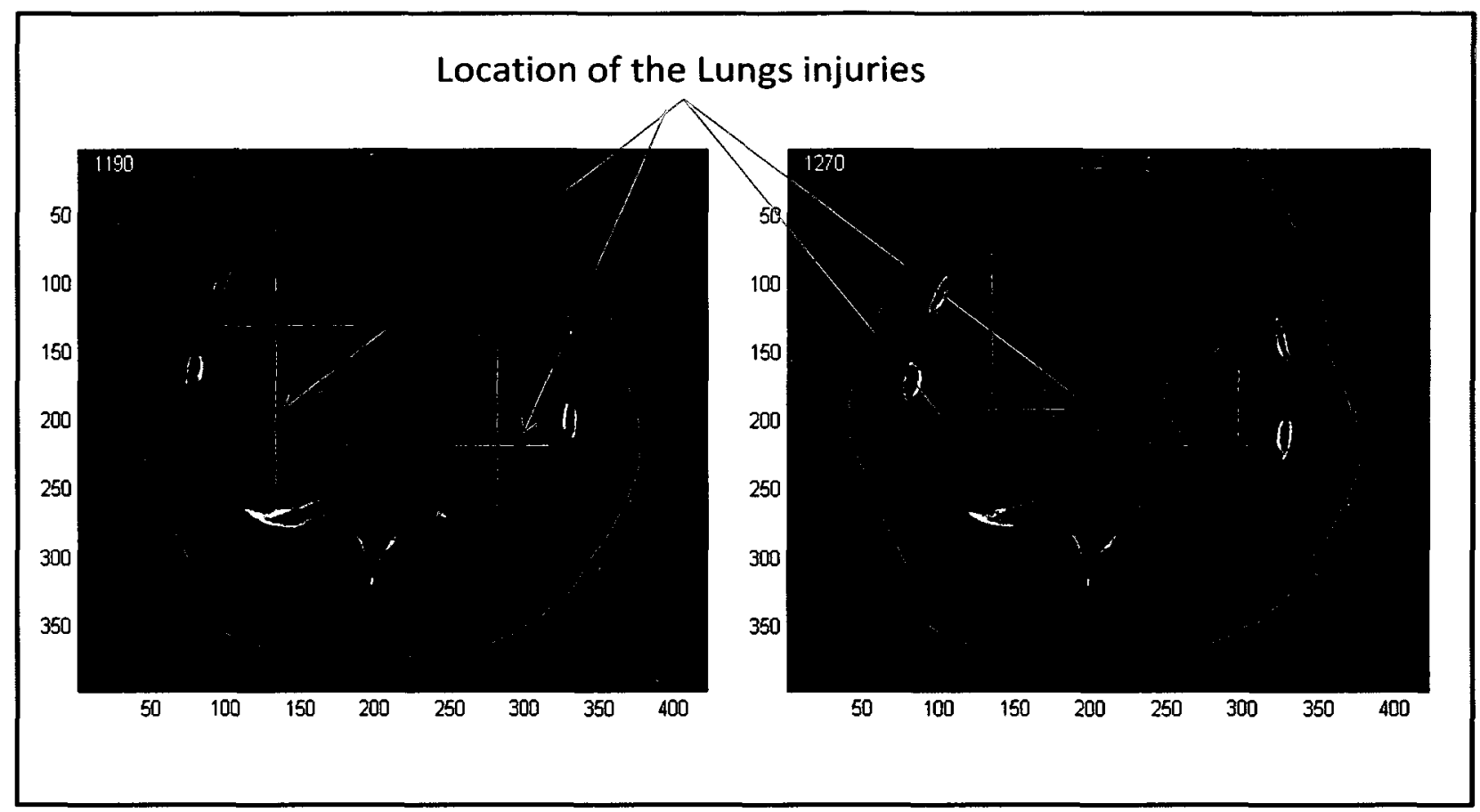

Figure 4- 6: CT images of a chest cross section of ALI diseased pig labelled "ards22-03", (left) air filled lungs (Paw $=40 \mathrm{cmH} 2 \mathrm{O}$ ), (right) air emptied lungs $(\mathrm{Paw}=0 \mathrm{cmH} 2 \mathrm{O})$. Lines indicate change in lungs size. Note the change in lung size and chest vertical dimension as well as the change in shape of the heart and the movement of air and blood vessels. The circled areas showing the result of injured lungs, before and after air emptying.

As seen in figure 4-6 (left), cross sectional CT image of the same ALI diseased pig's lungs before air emptying. It shows inflated lungs and bigger boundaries as an indication of air filled lungs.

The right image in the same figure shows a cross sectional CT image of the same pig's lungs after the air emptying. The lungs changed in size and shape as a result of increase in vertical and horizontal dimensions. The blue circles at the bottom of the lungs indicate the inflammation, infiltrations and lungs injuries as a result of ALI disease. 
Dynamic CT images of the lungs for the sample pig labelled "ards22-03" were analyzed through different steps. The following (figure 4-6) is an overall flowchart of the proposed algorithm to analyse dynamic CT images. 


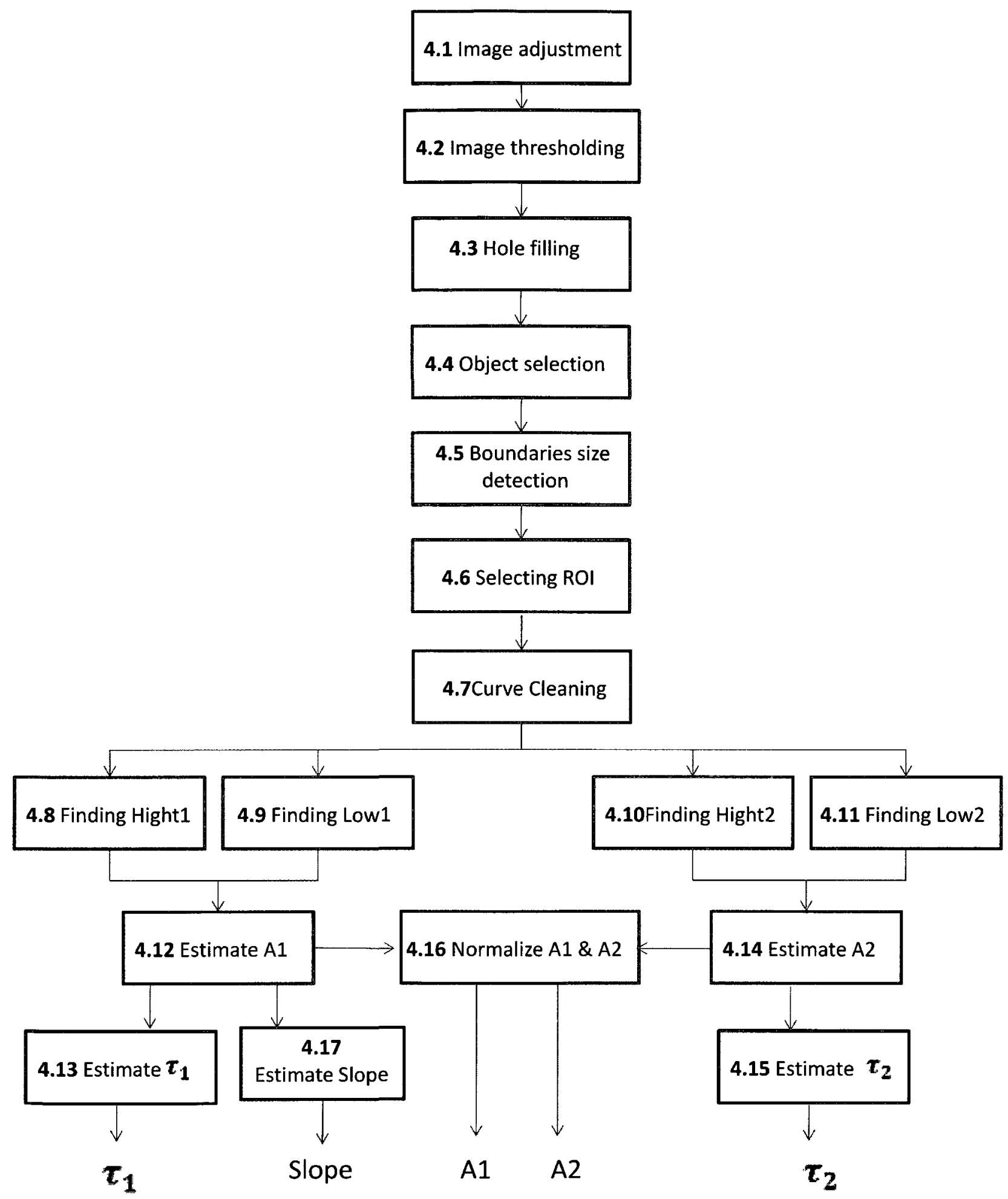

Figure 4- 7: A flowchart illustrating the algorithmic steps implemented to analyse CT data 
As seen in figure 4- 7, CT data are analyzed through 17 steps implemented to process CT images and estimate the 5 research parameters in step $4.13,4.17,4.16$, and 4.15 . Each step is explained in details below.

\subsection{Image adjustment:}

This step is to adjust the CT image obtained by DICOM read, MATLAB implemented method for reading image data from a compliant DICOM file obtained from a CT machine. It is needed to be able to visualize the changes in lungs shape and size, and track the lungs motion during a ventilation process. The adjustment of the image is done by deleting columns and rows from the left, bottom, and right of the image to have a cross sectional image of the chest, boundaries balanced with the edges of the image.

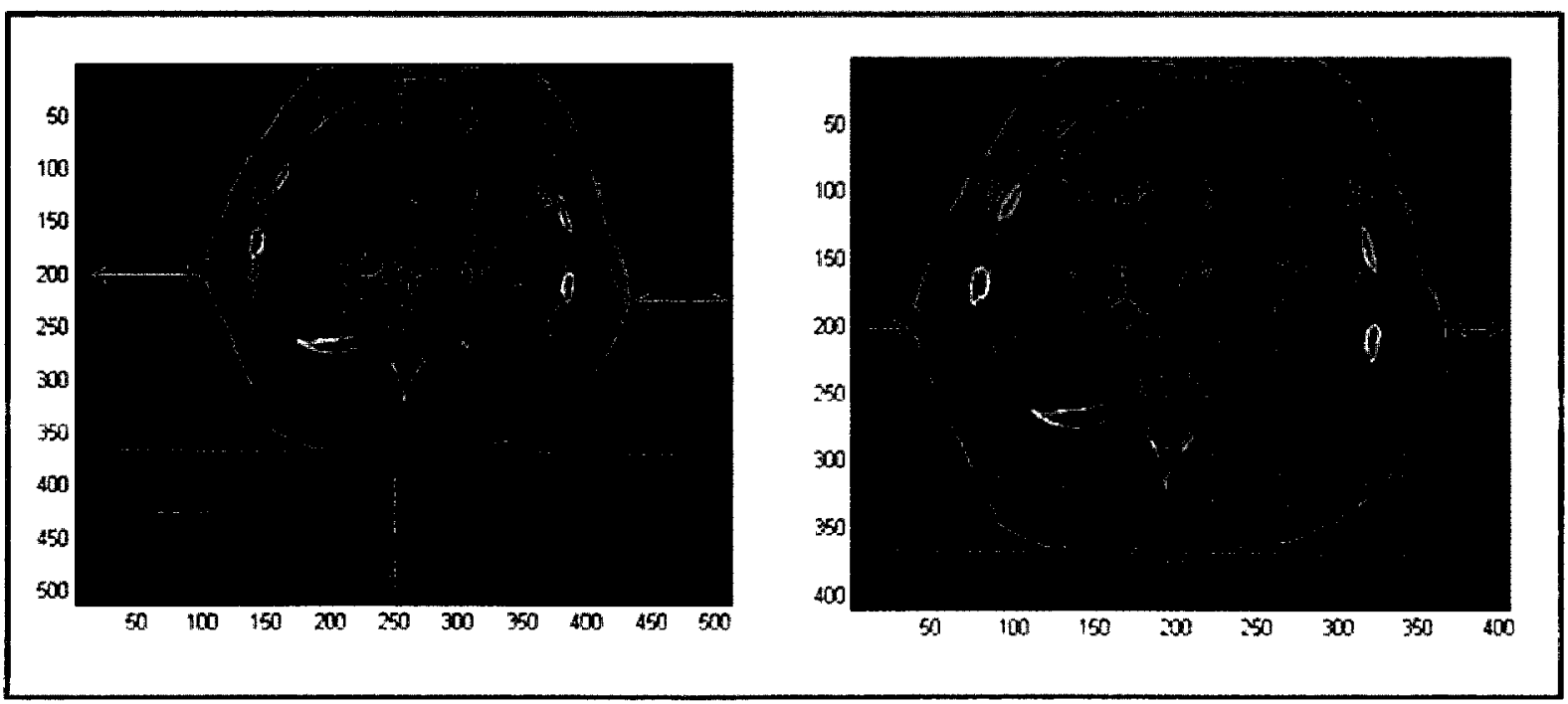

Figure 4- 8: (left) cross sectional CT image of the chest before image adjustment step, (right) the same CT image of the chest after image adjustment step. The distance between the image edges and the thorax boundaries, left, bottom, and right are balanced. 
As seen in figure 4-8, the left image is a CT cross section image of the chest before applying the image adjustment step. The chest boundaries, left, bottom, and right are not balanced with the image's edges.

The right image in the same figure shows the same CT image after image adjustment step is applied. The chest's boundaries, left, bottom, and right are balanced with respect to the image's edges.

\subsection{Image thresholding:}

The image pixel's values were converted into black (0) and white (1) using Otsu's method (Otsu, 1979) which chooses the threshold to minimize the intra-class variance of the black and white pixels. Pixels above the threshold are set to 1 and those which fall below are set to 0 as seen in figure 4-9 below. The level of threshold is a normalized intensity value that lies in the range $[0,1]$. The MATLAB graythresh command was used to perform the threshholding. 


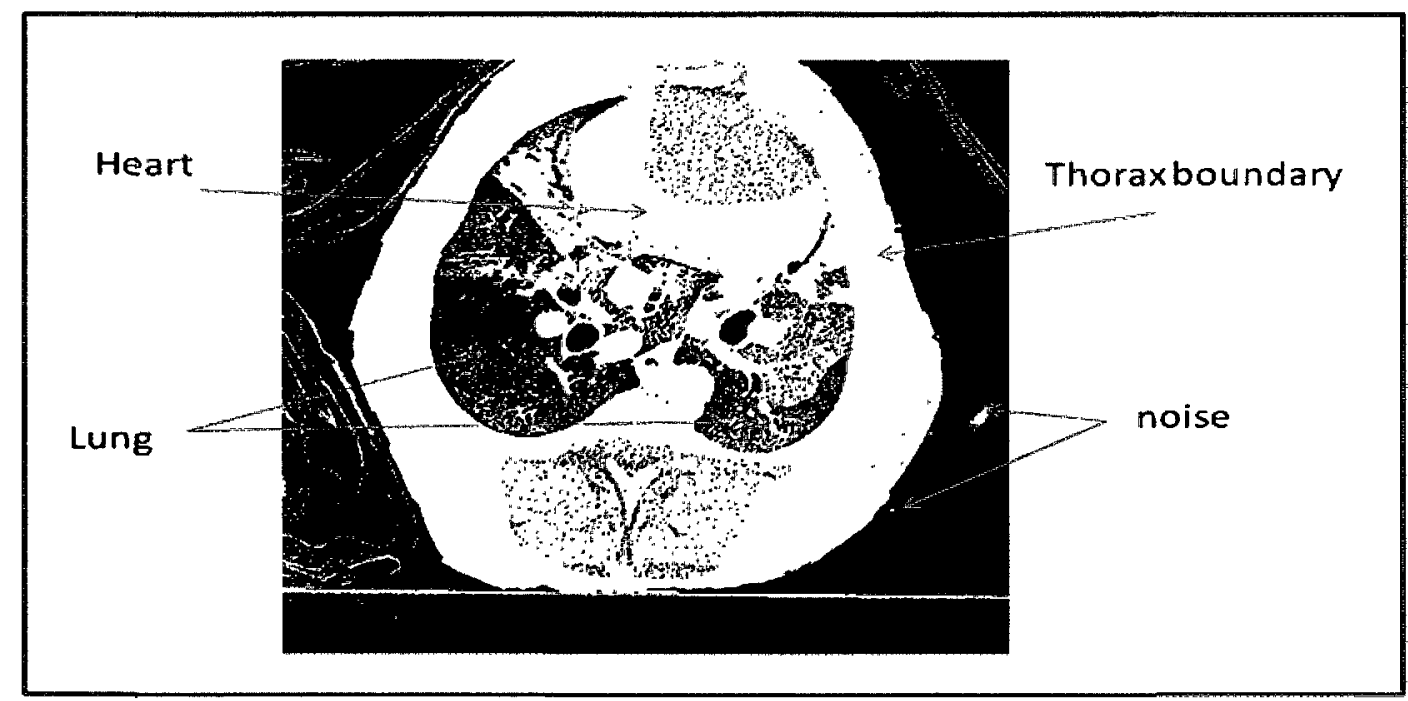

Figure 4- 9: 4CT image of a cross section of the chest for a sample pig after applying image thresholding step, illustrating the main objects in the image, thorax boundary, lungs, and heart.

As seen in figure 4- 9, CT cross section image of a sample pig's chest. It is a black and white image based on using the gray threshold. Regions with pixel values less than the gray threshold were set to 0 . Regions such as the heart and the thorax boundaries have higher pixel values in the image, these regions are set to 1.

\subsection{Hole filling:}

The image obtained from the previous step, 4.2 image thresholding, has the thorax boundary object and regions inside it, such as the lungs and heart. Therefore, considering the thorax boundary as one object, black regions inside the thorax boundary are considered as holes. This step fills the holes in the main object, thorax boundary, by converting each 0 pixel value inside the thorax into 1 . The MATLAB imfill command was used to fill the holes in the image obtained from the previous step (figure 4-9). It fills the holes in the binary image considering a 
hole as a set of background pixels that cannot be reached by filling in the background from the edge of the image.

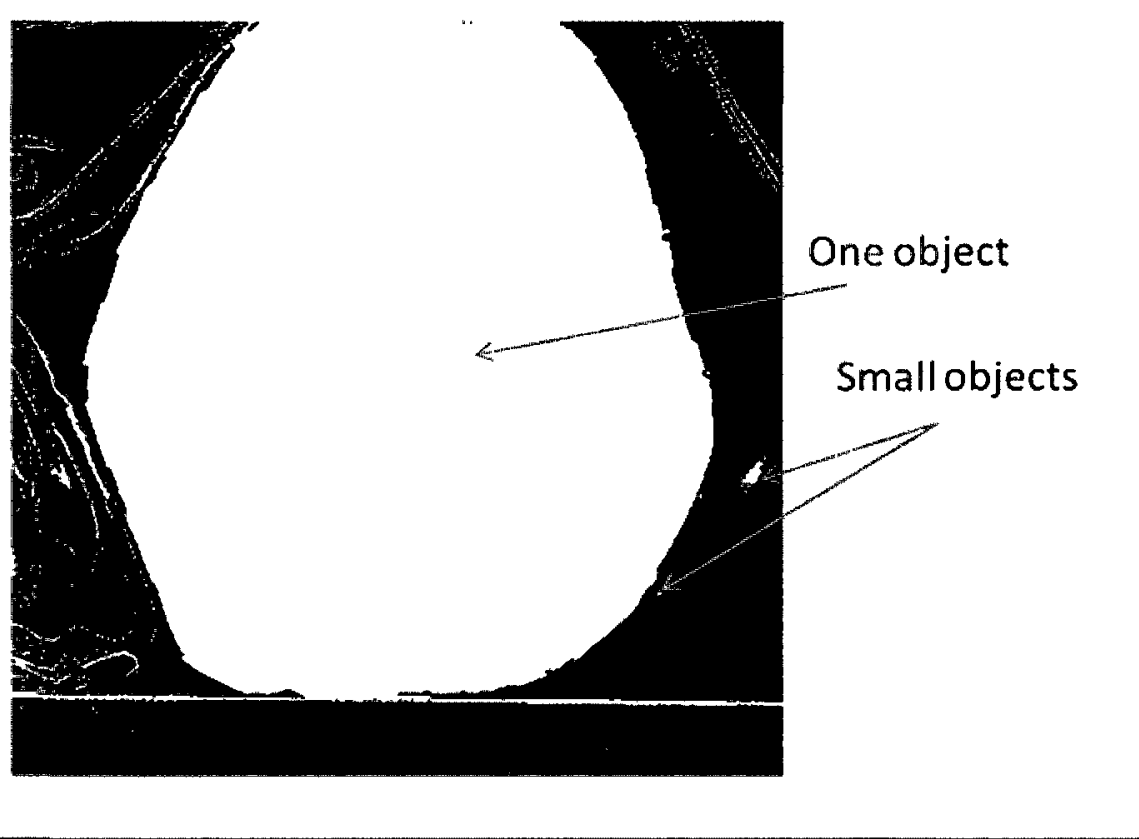

Figure 4- 10: cross sectional CT image of the chest after applying the hole filling step. Regions inside the thorax boundary changed into white colour (pixel value $=1$ ) and there is one object in the image representing the thorax boundary, lungs, and heart.

As seen in figure 4- 10, cross sectional CT image of the chest showing one object only representing thorax boundary, lungs, and the heart. There are other small objects in the image considered as noise.

\subsection{Object selection:}

This step finds the biggest object under investigation as a region of interest by eliminating all the small objects around boundaries and cleaning the images from any noise other than the largest object. The MATLAB imopen command was used in this step. It performs 
morphological opening on the binary image with a structuring element chosen to a matrix of ones. The morphological open operation is erosion followed by dilation.

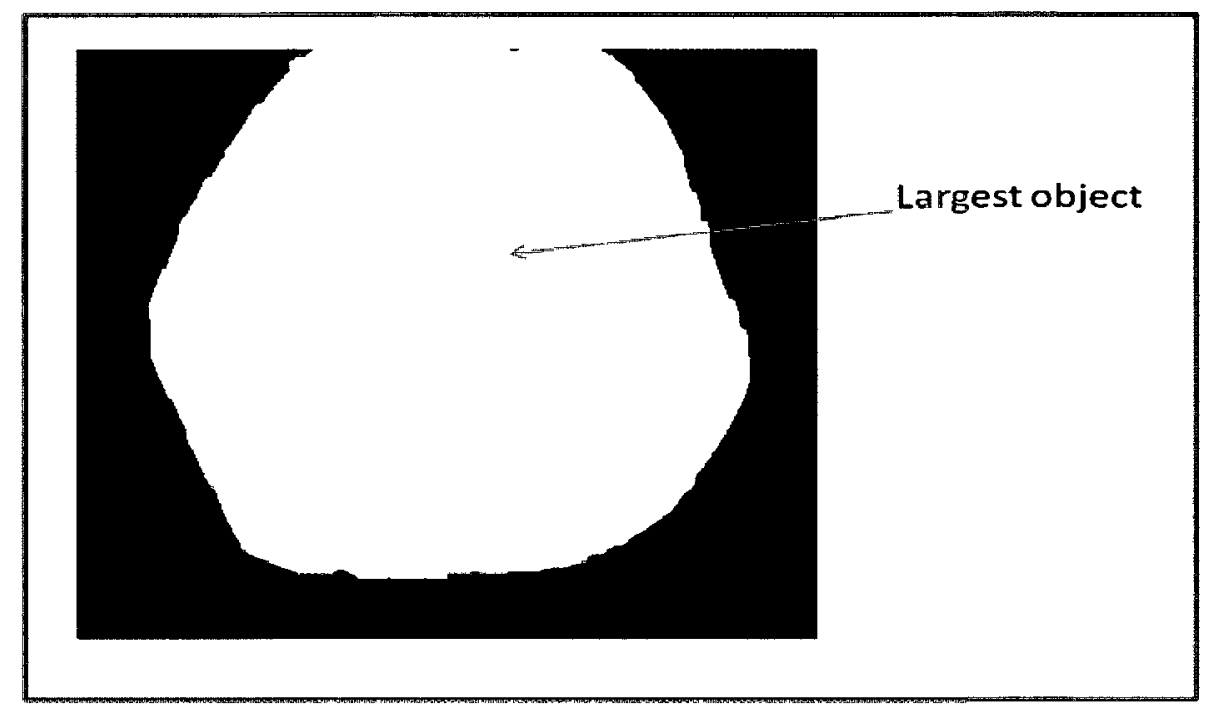

Figure 4- 11: CT image cross section of the chest after applying the main object selection step. There is only one main object representing the thorax boundary, lungs, and heart.

As seen in figure 4-11, the main object in the image is the largest object which represents the chest boundaries including thorax boundary, lungs, and the heart as one main object. Other small objects were cleaned from the image.

\subsection{Boundaries size detection:}

A reference point on the lungs is chosen for this step. A human operator locates the reference point on the lungs where vertical and horizontal boundaries change during ventilation. The distance from the chosen reference point in the vertical direction (Yi) to the upper boundary is captured. The same process is applied to the horizontal direction (Xi). The result of this step is obtaining a dynamic distance in both horizontal and vertical directions (figure 4- 12). 
This step is needed to detect the change of lung's size during ventilation applied. It is important to accurately locate ROIs selected while the lungs change in size.

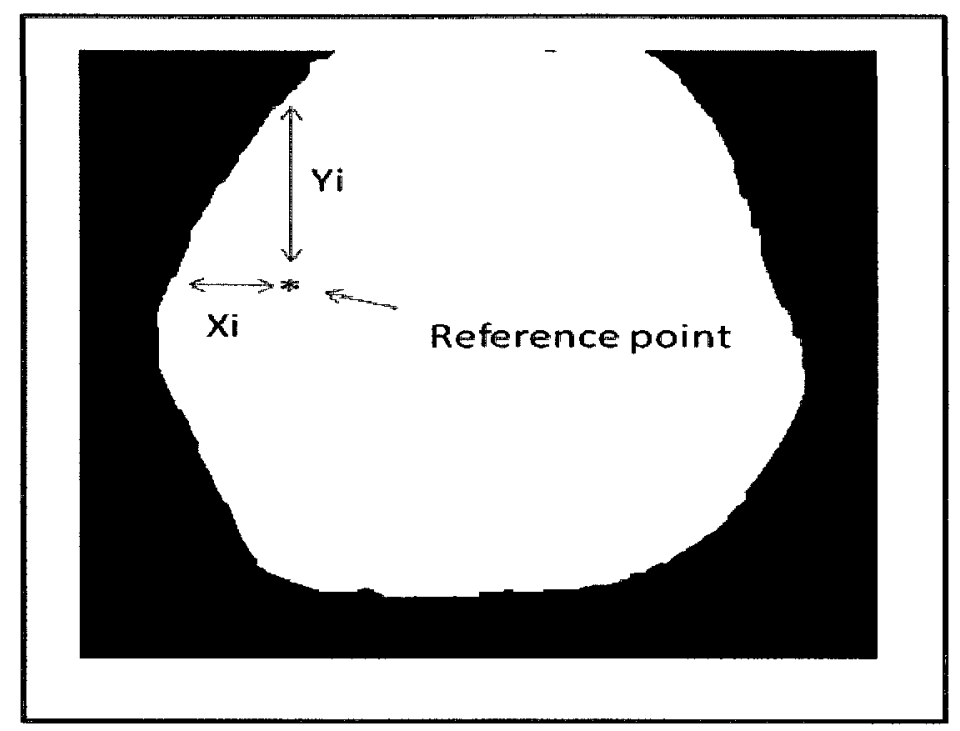

Figure 4- 12: cross sectional CT image of the sample pig's chest showing the reference point and the dynamic distances Xi and Yi obtained.

As seen in figure 4- 12, a reference point is chosen on the lungs. Vertical and horizontal distances $\mathrm{Yi}$ and $\mathrm{Xi}$ are detected on each image frame. The process is repeated for all the images during ventilation applied, air filling and air emptying.

\subsection{Selecting ROI}

A square shape representative selection of regions of interest, ROIs, was chosen on the lungs. It contains 20 ROIs (5 rows of 4 ROIs) with a 5 pixels apart. The coordinate of the representative selection of ROIs is chosen by the human operator by specifying the numbers of row and column on the image where the representative selection takes place. It is chosen to be on 
the lungs and away from the heart and the boundaries to make sure that the behaviour of each ROI is based on the ventilation process applied, air filling and air emptying (figure 4-12).

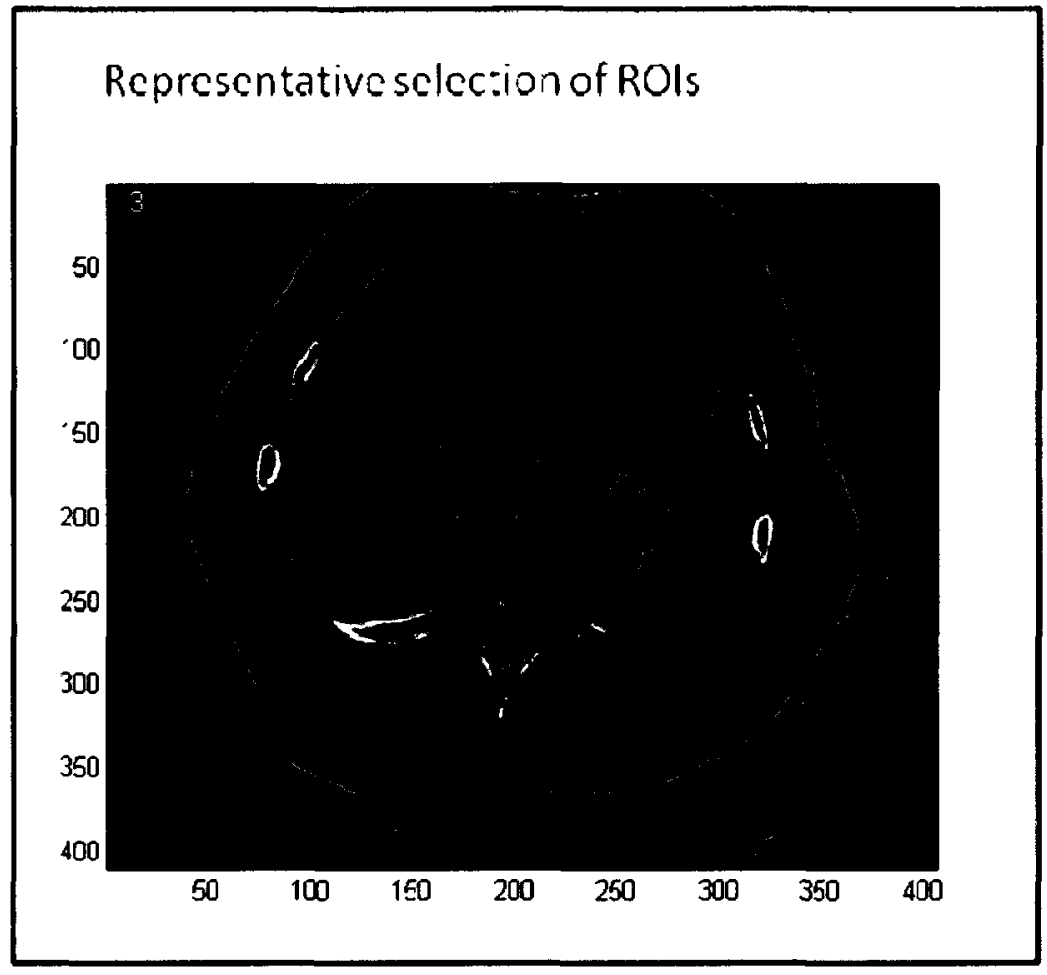

Figure 4- 13: cross sectional CT image of the sample pig's chest showing a representative selection of ROIs chosen to be on the lungs. Each blue '*' represent a single ROI.

As seen in figure 4- 13, cross sectional CT image of the sample pig's chest showing a representative selection of 20 ROIs selected on the lungs. Each ROI represents the summation of 5 pixel row values in 5 columns and then divided by 25 based on the following equation 4.1 .

$$
R O I_{2}=\frac{\sum_{m=1}^{\overline{5}} \Sigma_{n=1}^{\bar{s}} I(m, n)}{25}
$$


Where is the value of selected ROI, $i$ is the number of ROI from 1 to $20, m$ equals the row number, $n$ equals the column number, and $I(m, n)$ is the image pixel value at row $=m$ and column $=n$.

The coordinate of each ROI selected on the lungs changes with the motion of lungs when they inflate and deflate. $X i$ and $Y i$ obtained in step 4.5 before are used to obtain a new coordinate with respect to the lungs motion based on the following equations 4.2 , and 4.3.

$$
\begin{aligned}
& x^{\prime}=x \frac{X_{i}}{X_{i-1}} \\
& y^{\prime}=y \frac{Y_{i}}{Y_{i-1}}
\end{aligned}
$$

Where $x^{\prime}$ and $y^{\prime}$ are the new calculated coordinates of a ROI in the image row and column directions, $x$ and $y$ are the chosen coordinate of a ROI in the row and column directions, $X_{i}$ and $Y_{i}$ are the calculated horizontal and vertical distances at the current frame (detected in the previous step 4.5), and $X_{i-1}$ and $Y_{i-1}$ are the calculated horizontal and vertical distances in the previous frame (detected in the previous step 4.5).

Different representative selections of ROIs were chosen on the lungs left and right. The process is repeated for each ROI selected on each CT image frame. All ROIs data obtained through the whole CT image frames were processed to estimate the 5 research parameters (figure 4- 14). 


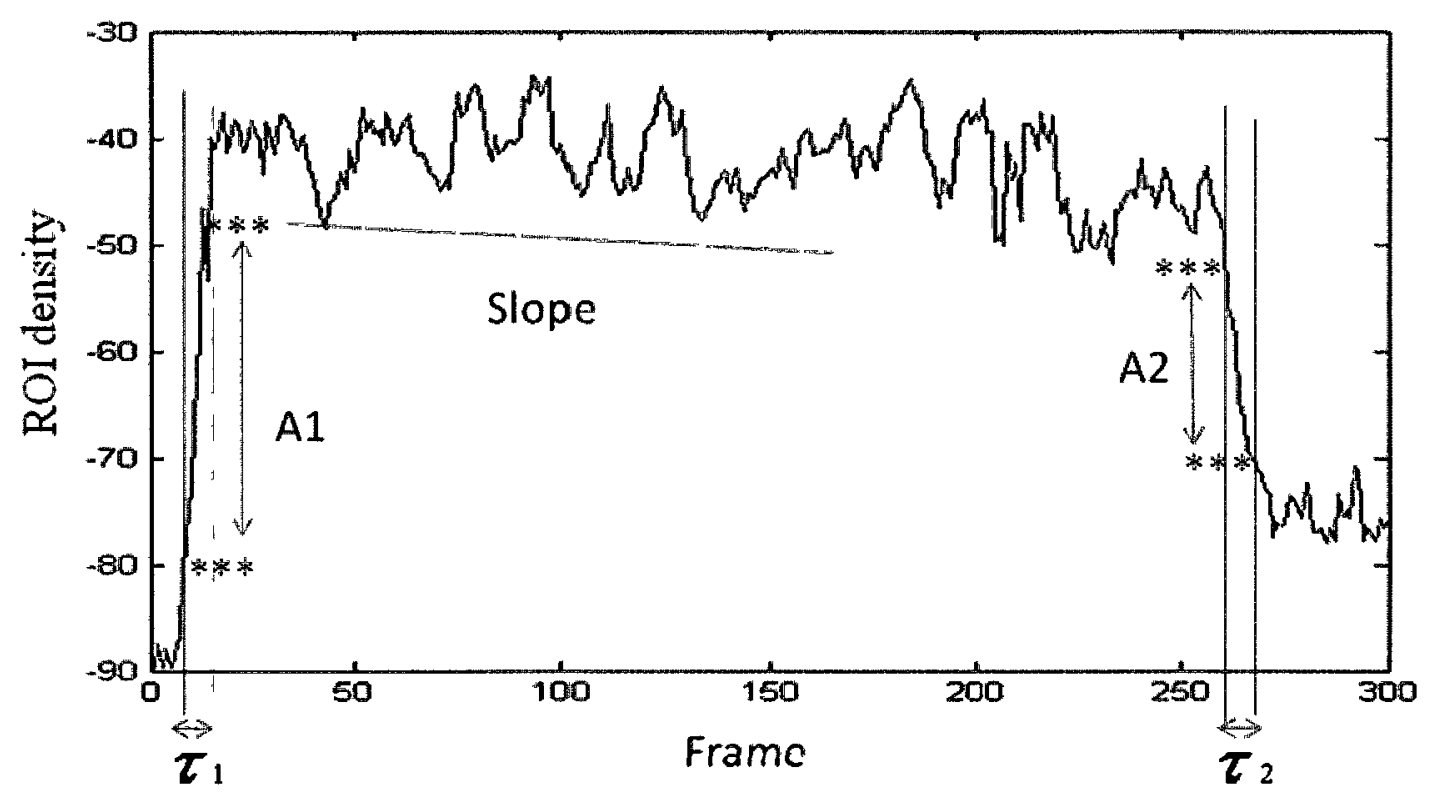

Figure 4- 14: Density versus frame number for a ROI selected on dynamic CT image of the sample pig. The 5 research parameters $\mathrm{A} 1, \tau_{1}$, Slope, $\mathrm{A} 2$, and $\tau_{2}$ are shown on the graph obtained.

As seen in figure 4- 14, density versus frame number for a single ROI selected on dynamic CT image of the sample pig's lungs. The graph shows the 5 research parameters need to be estimated on both dynamic CT and EIT images. The following define each parameter on the graph.

$\tau_{1}$ is the time it takes for a selected ROI on the lungs to be filled with air.

A1 is the amplitude in density for a selected ROI on the lungs from the start to the end of air filling.

Slope is the value of the steepness of the line between air filling and emptying.

$\tau_{2}$ is the time it takes for a selected ROI on the lungs to be emptied from air. 
A2 is the amplitude in density for a selected ROI on the lungs from the start to the end of air emptying.

\subsection{Curve Cleaning:}

Graphs obtained from each ROI selected on the dynamic CT images require curve cleaning because of noise and contain periodic heart beat signals. The closer the ROI to the heart and boundaries, the noisier the graph obtained. This step is needed to eliminate edge sensitivity which may affect the estimation of research parameters (figure 4- 15).

The original data curve obtained was cleaned from noise by finding the coefficients of a polynomial function $\mathrm{P}(\mathrm{X})$ of degree $\mathrm{N}$ (e.g., $\mathrm{N}=15$ ) that best fits the ROI's $\mathrm{CT}$ image data curve. The MATLAB polyfit function was applied to the data curve to obtain data best fit curve and eliminate the noise. The MATLAB polyval function was used as an algorithm to evaluate the obtained coefficients at all points of a vector X equal to the length of the ROI's CT data curve (figure 4- 15). 

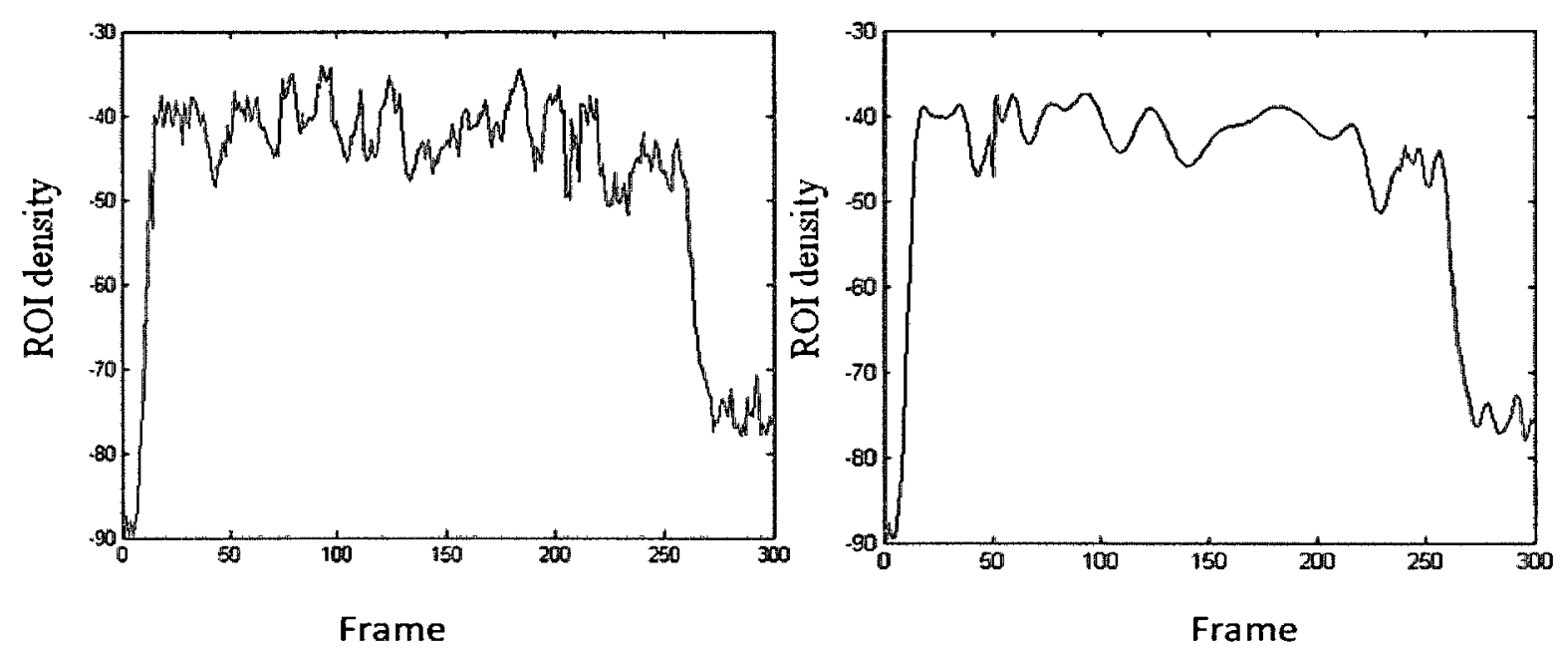

Figure 4- 15: (left) density versus frame number for a selected ROI on the lungs before applying the curve cleaning step, (right) density versus frame number for the same ROI after applying the curve cleaning step.

As seen in figure 4- 15, left is a selected ROI density versus frame number graph before applying the curve cleaning step, (right) is the same graph after applying the curve cleaning step. The curve has less noise and is not sensitive to the e dges where the research $p$ arameters a re estimated.

\subsection{Finding High1:}

This step is to find the highest point represent the end of air filling on the curve. The highest point is found by iterating through the first half of the curve and finding the maximum point on the graph (figure 4-16). 


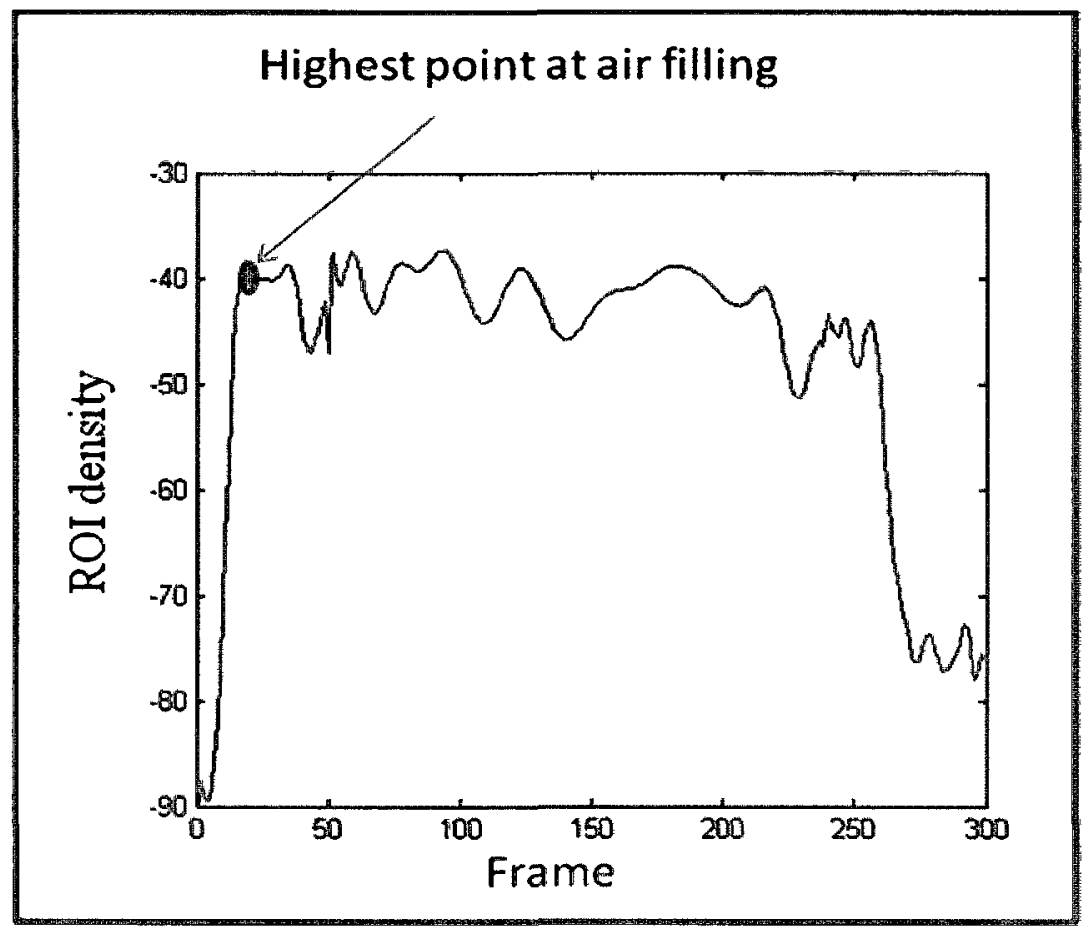

Figure 4- 16: Density of a single ROI versus frame number curve obtained from CT images of the lungs, it shows a blue circle indicating where the highest point found on the curve during air filling.

As seen in figure 4- 16, density versus frame number curve for a selected ROI on the CT image of the sample pig's lungs showing the highest point found during air filling. The blue circle indicates the location on the graph where the "highl" point was found. The estimated highest point found is obtained for all the ROI and will be used in the next analysis steps.

\subsection{Finding Low1:}

This step is to find the lowest point on the $\mathrm{c}$ urve that $\mathrm{r}$ epresents the $\mathrm{s}$ tart of 1 ung a ir filling. The lowest point is found by iterating and finding the minimum point through the first half of the curve and before high1 (figure 4-17). 


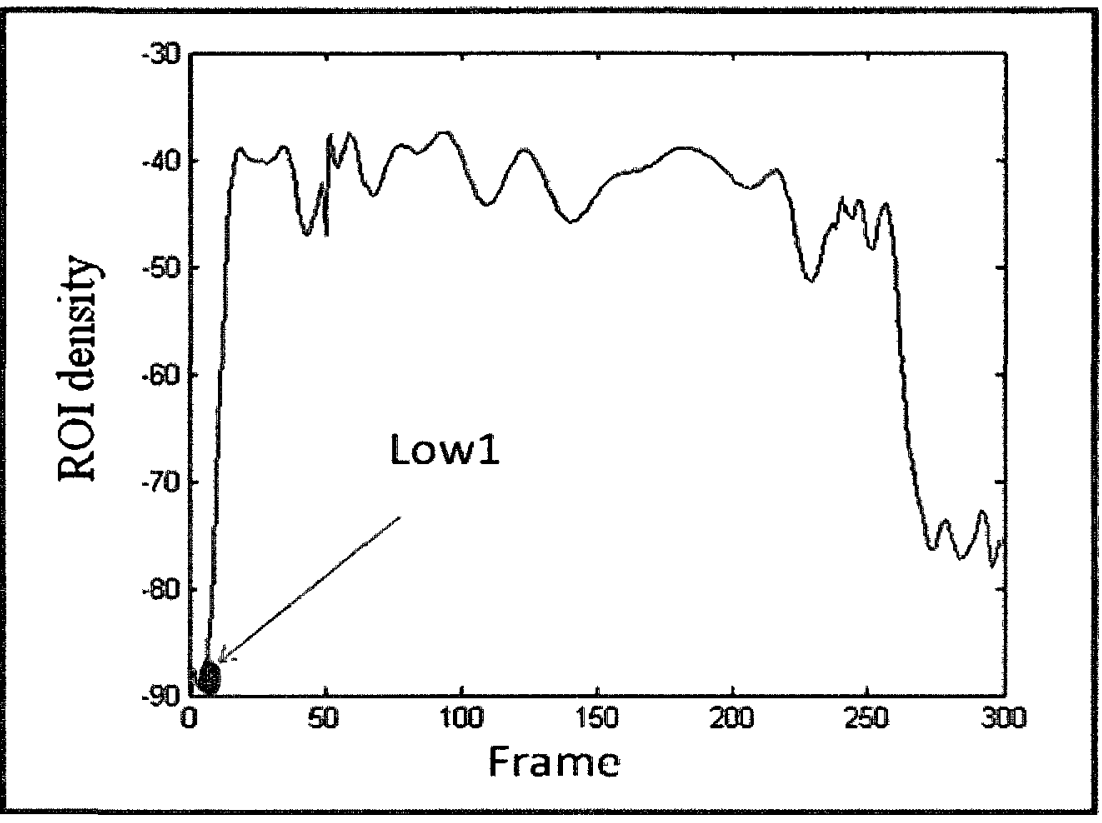

Figure 4- 17: Density of a selected single ROI versus frame number curve of an EIT image of the lungs, it shows a blue circle indicating where the lowest point found on the curve during air filling.

As seen in figure 4- 17, density versus frame number curve for a selected ROI on the CT image of the lungs showing the lowest point found before air filling. The blue circle indicates where low1 point found on the curve. The estimated lowest point found was obtained for all the ROI and will be used in the next analysis steps.

\subsection{Finding High2:}

This step is to find the highest p oint on the curve that r epresents the start of l ung air emptying. The "high2" point is found by iterating through the s econd half of the curve a nd finding the maximum point before the curve drops down (lungs get air emptied) (figure 4- 18). 


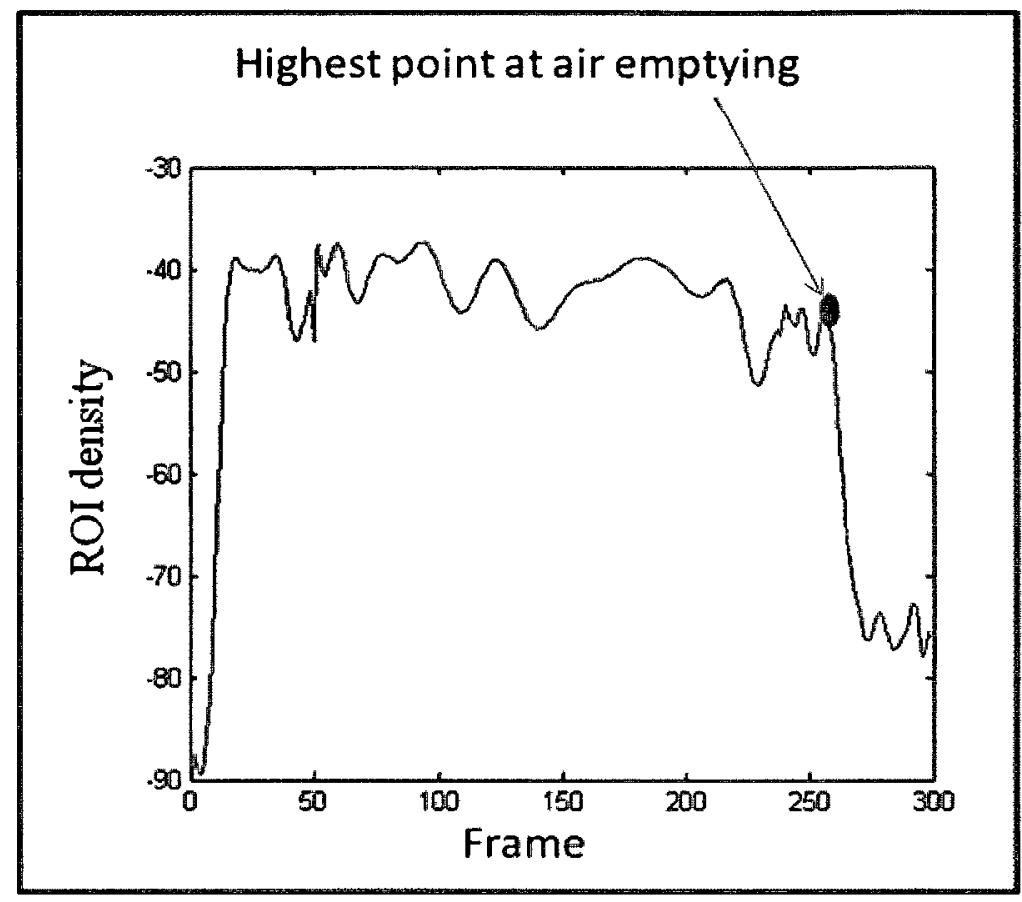

Figure 4- 18: Pixel value of a selected single ROI versus frame number curve obtained from a CT image of the lungs, it shows a blue circle indicating where the highest point found on the curve at the start of air emptying.

As seen in figure 4- 18, density versus frame number curve for a selected ROI on CT image of the lungs showing the highest point found at the start of air emptying. The blue circle indicates where the "high2" point found on the curve. The e stimated h ighest p oint found is obtained for all the ROI and will be used in the next analysis steps.

\subsection{Finding Low2:}

This step is to find the lowest point on the curve that represents the end of 1 ung air emptying. The lowest point is found by i terating and finding the minimum point through the second half of the curve and after "high2" (figure 4- 19). 


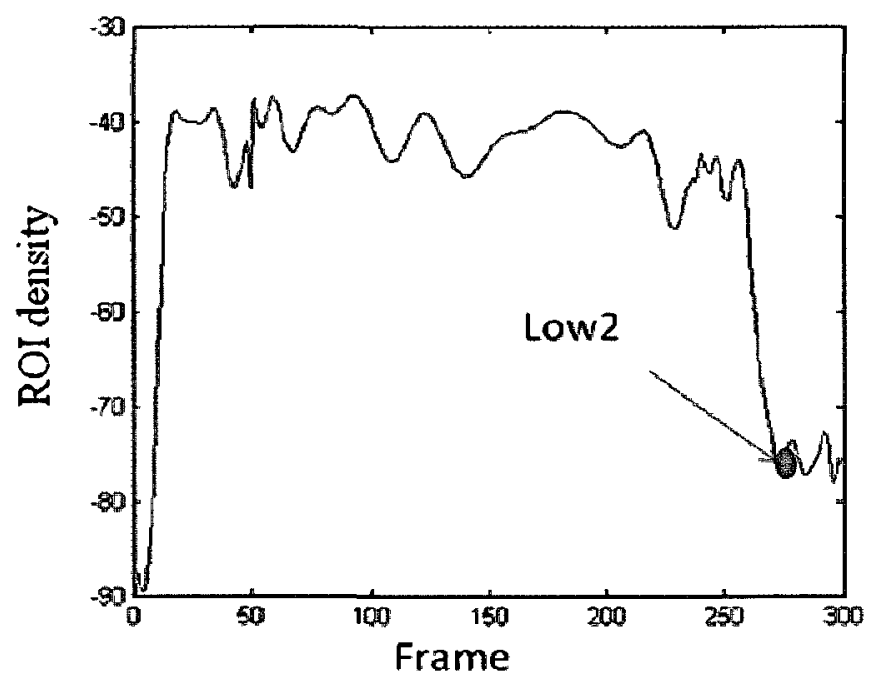

Figure 4- 19: Density of a selected single ROI versus frame number curve of a CT image of the lungs, it shows a blue circle indicating where the lowest point found on the curve during air emptying.

As seen in figure 4- 19, density versus frame number curve for a selected ROI on CT image showing the location of the lowest point on the curve. The blue circle indicates the lowest point when the curve drops down at the end of air emptying. The estimated lowest point found is obtained for all the ROI and will be used in the next analysis steps.

\subsection{Estimating A1:}

This step is to estimate the research parameter A 1 on the density versus frame number curve obtained from ROI selected on CT images of the lungs. The process of this step uses the "highl" and "low1" points found on the air filling curve obtained in steps 4.8, finding "high1", and 4.9, finding "low1". 
A1 is estimated by iterating through the $\mathrm{c}$ urve t o f ind new highest and lowest points which are new values of highl and lowl after eliminating $20 \%$ of each. This process is applied to avoid edge curve sensitivity, the start and end of air filling on the curve (figure 4- 20).

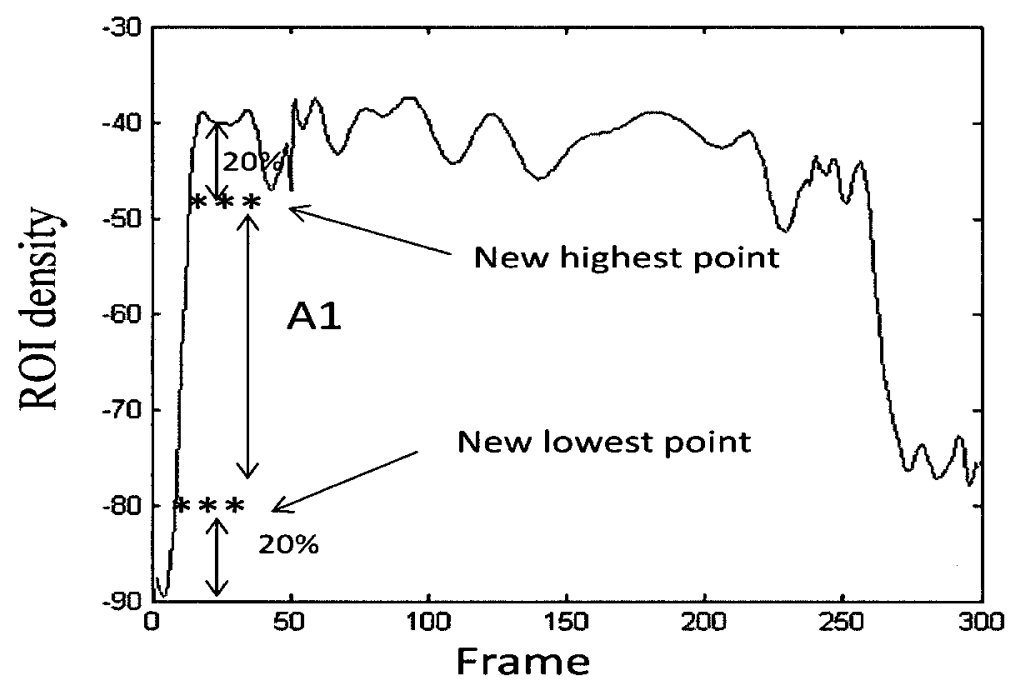

Figure 4- 20: Density of a selected single ROI versus frame number curve of a CT image of the lungs, it shows the new highest and lowest points "***" found after eliminating $20 \%$ of the original highest and lowest points found before. Al is estimated from the new highest and lowest points for air filling.

As seen in figure 4- 20, density versus frame number curve for a selected ROI on CT image showing research parameter A1 estimated. The new highest and lowest points are detected on the curve by eliminating $20 \%$ of high 1 and low 1 values. The estimated research parameter A1 is shown on the curve and the process is repeated for all ROI data obtained.

\subsection{Estimating :}

This step is to estimate research $\mathrm{p}$ arameter $\tau_{1}$ on density versus frame number $\mathrm{c}$ urve obtained from a selected ROI chosen on CT images of the lungs. The process of this step uses the new highest and lowest points found in step 4.12, Estimating A1. 
Research parameter $\tau_{1}$ is estimated by finding the indexes of the new highest and lowest points on the curve (figure 4-21). Then, subtracting the index of the new lowest point from the index of the new highest point and the result is converted to seconds by dividing it by 10 (CT image frequency rate is $10 \mathrm{~Hz}$ ).

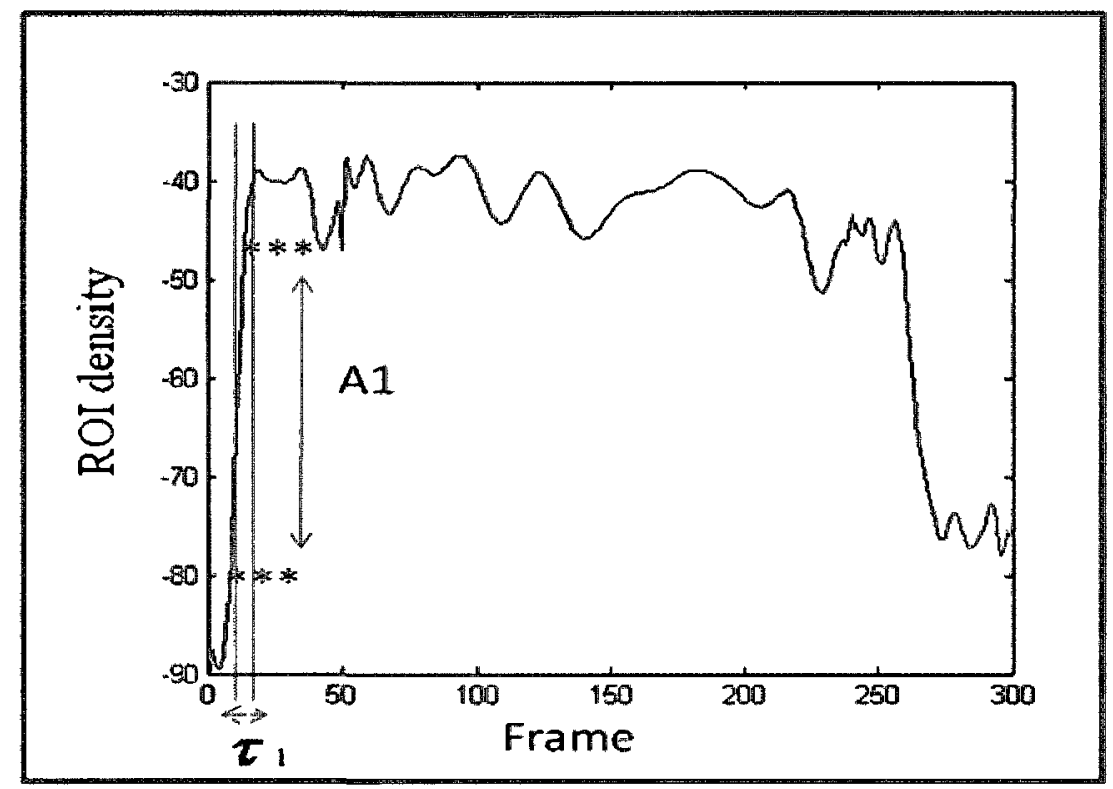

Figure 4-21: Density of a selected single ROI versus frame number curve of a CT image of the lungs, it shows the estimated research parameter $\tau_{1}$.

As seen in figure 4- 21, density versus frame number curve for a selected ROI on CT image showing the estimated research $p$ arameter $\tau_{1}$. The indexes of new highest and lowest points are detected to be used to estimate research parameter $\tau_{1}$ which is measured in seconds. 


\subsection{Estimating A2:}

This step is to estimate the research parameter $\mathrm{A} 2 \mathrm{on}$ the curve o btained from $\mathrm{R}$ OI selected on CT image of the lungs. The process of this step u ses the high 2 and 1 ow 2 points obtained in steps 4.10 , finding high2, and 4.11 , finding low2.

A2 is estimated by iterating through the curve to find new highest and lowest points which are new values of high 2 and low 2 after eliminating $20 \%$ of each. This process is applied to avoid edge curve sensitivity, the start and end of air emptying on the curve (figure 4- 22).

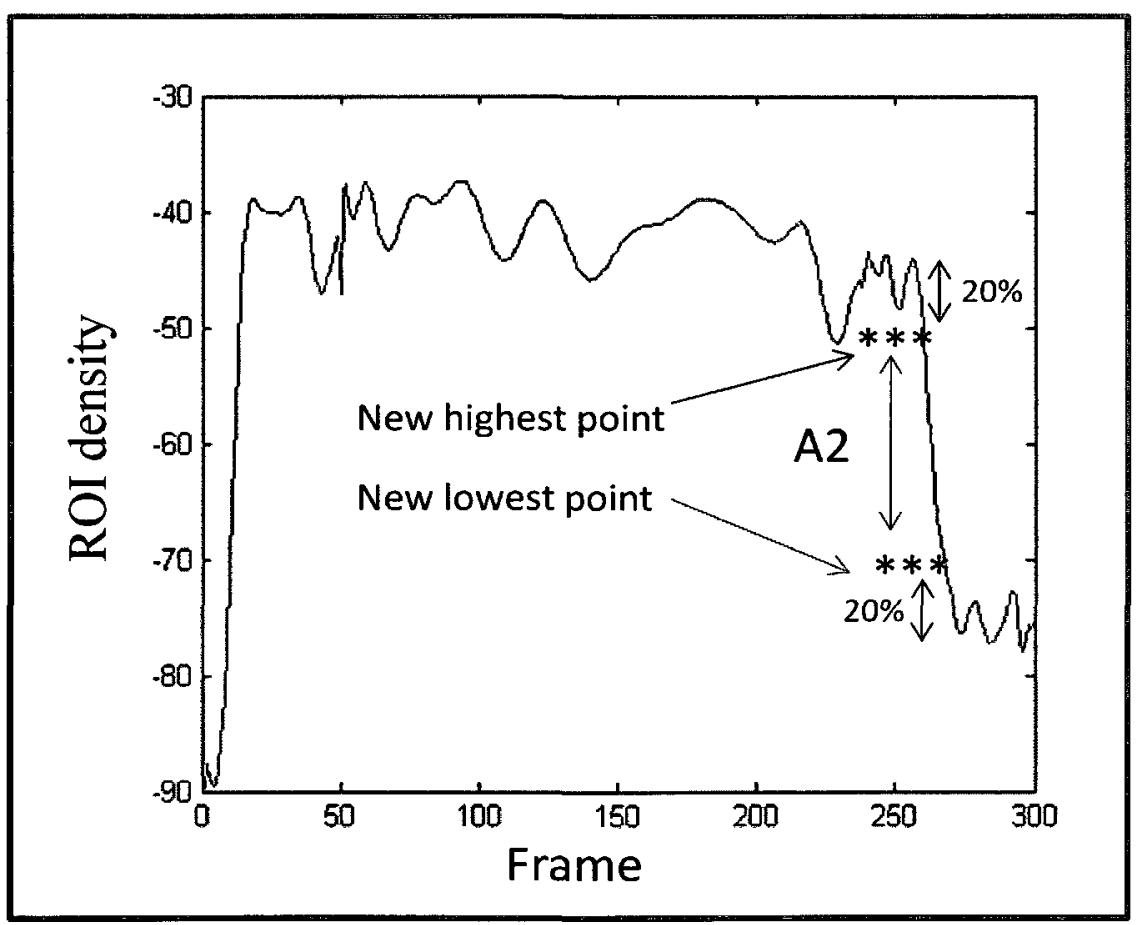

Figure 4- 22: Density of a selected single ROI versus frame number curve of a CT image of the lungs, it shows the new highest and lowest points "***" after eliminating $20 \%$ of high 2 and low2 points. Research parameter A2 is estimated from the new highest and lowest points during air emptying. 
As seen in figure 4- 22, density versus frame number curve for a selected ROI on CT image showing research parameter A2 estimated on the curve. $20 \%$ from high2 and low2 points were eliminated and new highest and lowest points are detected on the curve.

\subsection{Estimating :}

This step is to estimate research $\mathrm{p}$ arameter $\tau_{2}$ on density versus frame number $\mathrm{g}$ raph obtained from a selected ROI on CT image of the lungs. The process of this step uses the new highest and lowest points found in step 4.15.

Research parameter $\tau_{2}$ is estimated by finding the indexes of the new highest and lowest points on the curve. Then, subtracting the index of the new lowest point from the index of the new highest point and the result is converted into seconds by dividing it by 10 (CT i mage frequency rate is $10 \mathrm{~Hz}$ ).

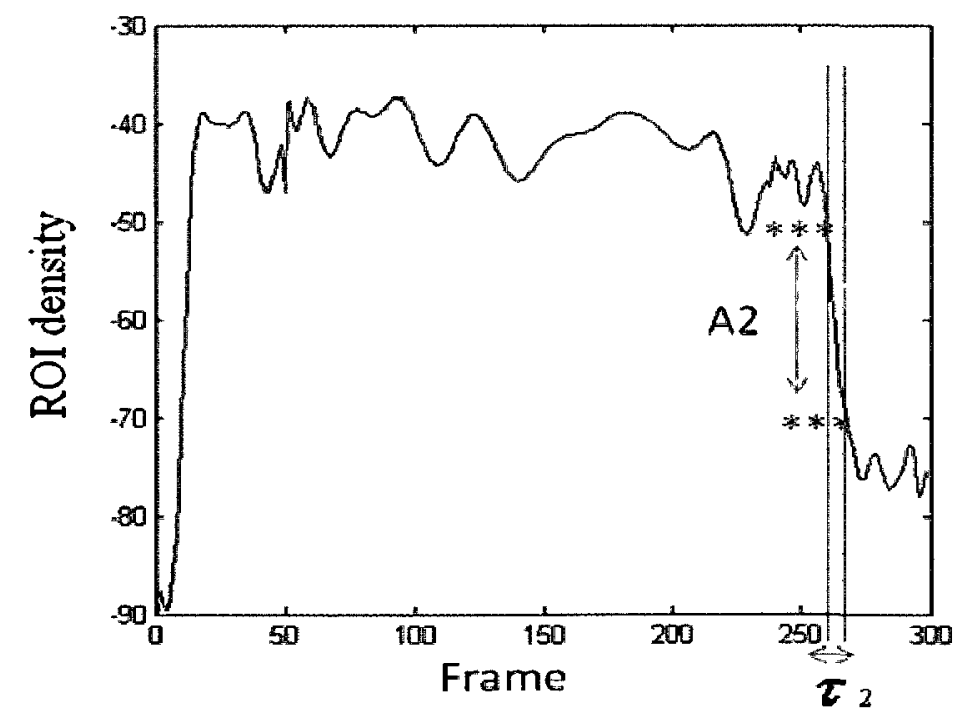

Figure 4- 23: Density of a selected single ROI versus frame number curve of an EIT image of the lungs. It shows the estimated research parameter $\tau_{2}$ for air emptying. 
As seen in figure 4- 23, research parameter $\tau_{2}$ is estimated on the graph obtained from a selected ROI on CT image of the lungs. The curve indexes of the new highest and lowest points estimated on the CT curve are considered in this estimation. $\tau_{2}$ is estimated in seconds and the process is repeated for all ROIs selected on the image.

\subsection{Normalizing A1 and A2:}

Research parameters A1 and A2 were normalized by dividing by the mean value of each set for all ROIs. This step is needed because research parameters A1 and A2 estimated on the CT image's ROIs vary from one to another. For example, A1 estimated on a graph obtained from ROI selected on the top of the lungs is higher than the same parameter estimated on ROI selected on the middle or on the bottom of the lungs.

\subsection{Estimating the Slope:}

This step is to estimate the slope of the curve located between high1 and high2 points, end of air filling and beginning air emptying. The best straight line was obtained in the curve between the two events air filling and air emptying (figure 4- 24). The MATLAB polyfit method, explained in step 4.7 , at degree $\mathrm{N}=1$ was used to obtain the best fit. 


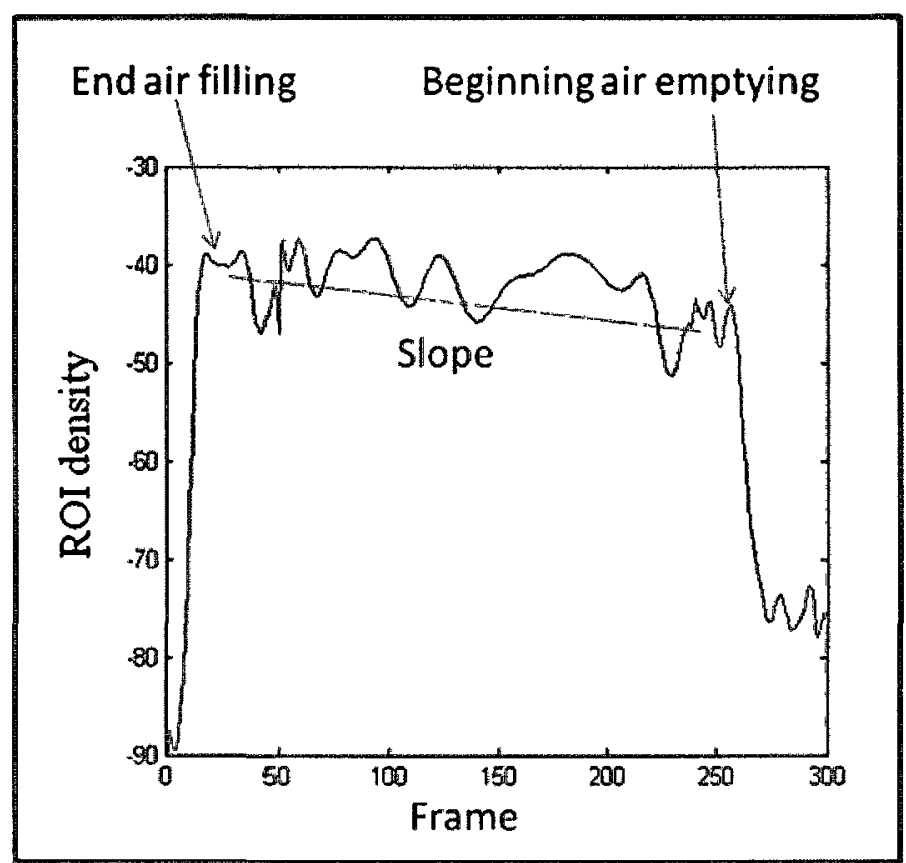

Figure 4- 24: Density versus frame number curve of a ROI selected on a CT image of the lungs. The green line on the curve shows the slope between end of air filling and beginning of air emptying.

As seen in figure 4- 24, the slope is shown on the curve between the end of air filling and beginning of air emptying. A straight line is best fitted into the curve. The process is repeated for all the ROI density versus frame number curves obtained from CT images of the lungs.

The slope is normalized to research parameter A1 by dividing each ROI's slope obtained by the mean value of research parameter A1 set estimated before. 


\section{Chapter 5}

\section{EIT Analysis:}

This Chapter explains the methodology developed to a nalyse the E IT i mage $d$ ata. It analyzes EIT images obtained from a selected pig. EIT data were analyzed during ventilation, air filling and then air emptying, applied to h ealthy and A LI diseased pigs. A lso, this s ection contains a block diagram showing the algorithm implemented to analyze the EIT data. Images and graphs describing the EIT analysis algorithm are shown as well.

EIT data were acquired by applying 50-mA alternative current to 16 electrodes placed around the circumference of a pig chest. Current was injected across each pair of electrodes and voltage measurements were obtained $\mathrm{f}$ rom all the e lectrodes g enerating $256 \mathrm{v}$ oltage measurements which are called data frame. A total of 1560 data frames were obtained within a period of two minutes.

Reference voltage value was calculated based on the mean value of all the measurements voltage obtained. The difference measurement, $\Delta V$ was obtained by subtracting the reference voltage, $V_{r e f} \cdot$ from each measured voltage value, $V_{i}$ and then normalized by dividing by the reference value, $V_{r s f}$.

The EIT difference image is reconstructed $\mathrm{b}$ ased on the linearized one-step difference EIT regularized image reconstruction algorithm according to the following equation (1). 


$$
\lrcorner \varepsilon_{i}=\left(J^{T} \cdot J-\lambda \cdot\right)^{-1} J^{T} \cdot \Delta V_{i}
$$

Where $\Delta \boldsymbol{\delta}_{\mathrm{i}}$ is the reconstructed difference image, $\boldsymbol{J}$ is the Jacobian matrix (sensitivity matrix), $f^{T}$ is the Jacobian matrix transpose, is the regularization parameter, $\mathrm{R}$ is the regularization matrix, $\Delta V$ is an array of obtained difference measured voltages, and $i$ is the number of frames, e. g, 1560 slices of difference images were reconstructed from 1560 data frames (Adler and Guardo, 1996).

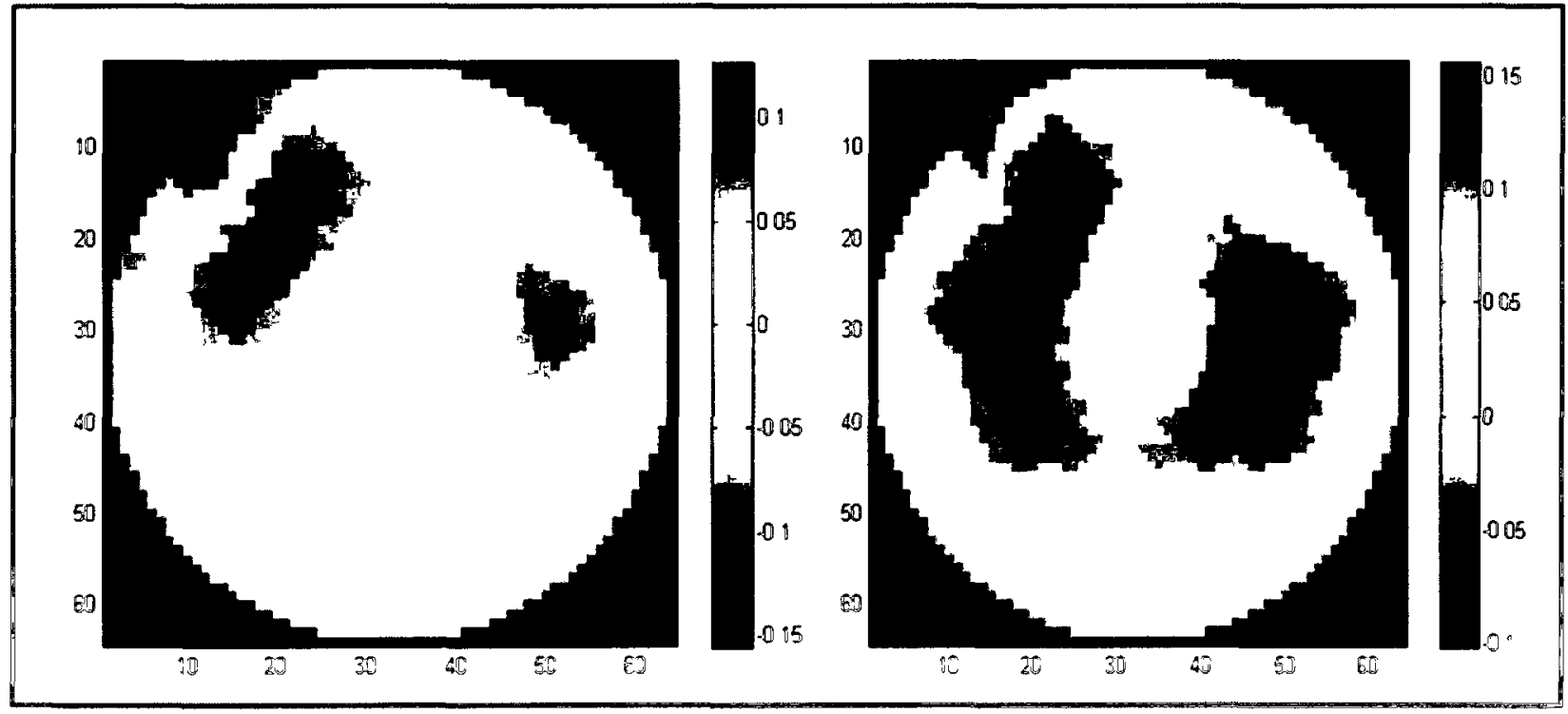

Figure 5-1: EIT images of a chest cross section of a healthy pig (no lung injury), (left) lungs before air filling (Paw $=0 \mathrm{cmH} 2 \mathrm{O})$, (right) lungs after air filling $(\mathrm{Paw}=40 \mathrm{cmH} 2 \mathrm{O})$. The status of air filled image chang based on the chosen difference point $(\quad)$.

As seen in figure 5- 1, (left) the EIT cross sectional image of the lungs for a selected healthy pig was obtained before air filling, and (right) is a cross sectional image of the lungs for 
the same pig after air filling. Both images before and a fter a ir filling, were changed in value based on the reference voltage ( ) explained before in equation 5-1.

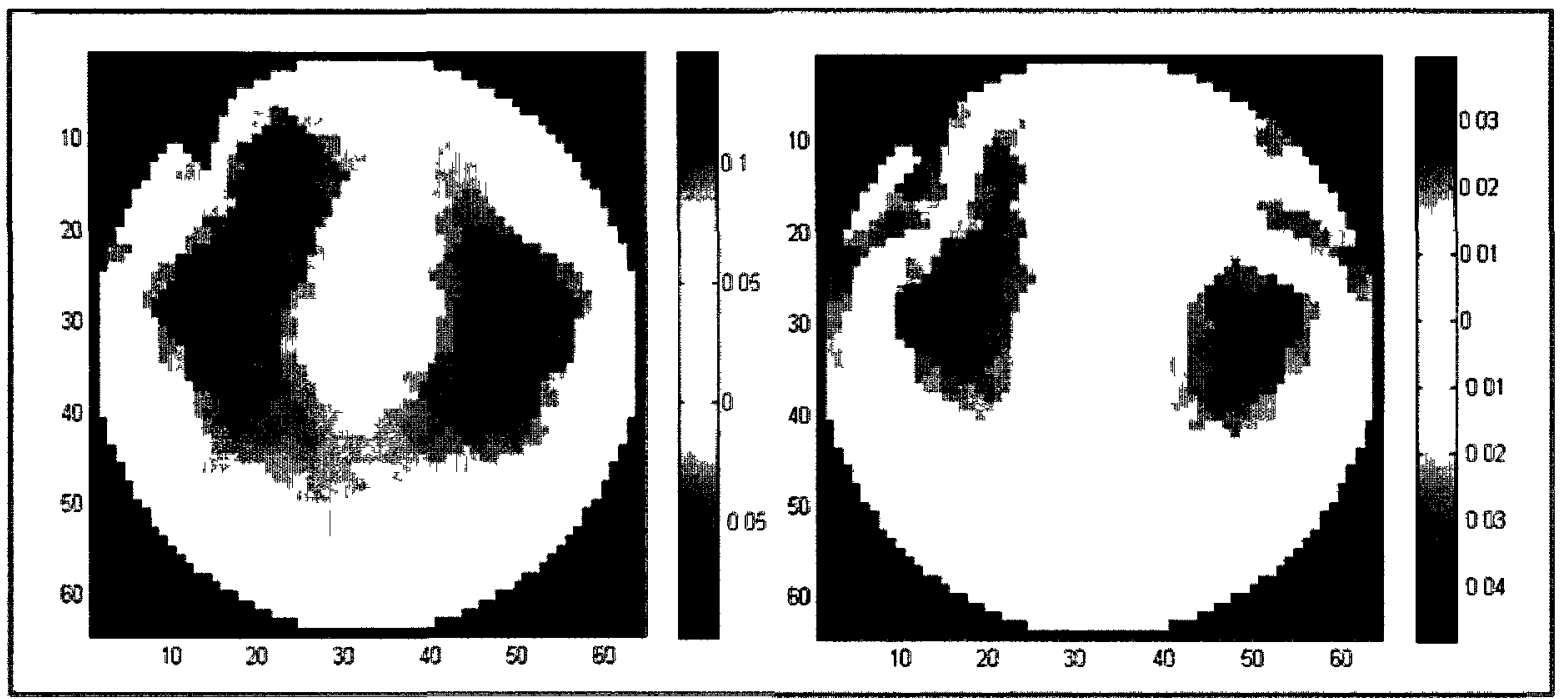

Figure 5- 2: EIT images of a chest cross section of a health pig (no lung injury), (left) lungs before air emptying $(\mathrm{Paw}=40 \mathrm{cmH} 2 \mathrm{O})$, (right) lungs after air emptying $(\mathrm{Paw}=0 \mathrm{cmH} 2 \mathrm{O})$. The status of air emptied image changes based on the chosen difference point ( ).

As seen in figure 5-2, the left image is an EIT cross sectional image of the lungs was obtained before air emptying. The right image is a cross sectional image of the lungs a fter air emptying. Considering the same reference point $\mathrm{c}$ hosen as $\mathrm{b}$ efore, the values of both i mages were changes.

Repeated lungs lavage was done for each pig causing ALI. Animals were ventilated again with the same scenarios, air filling and then air emptying. Meanwhile, EIT images of the lungs were taken over a period of time. 


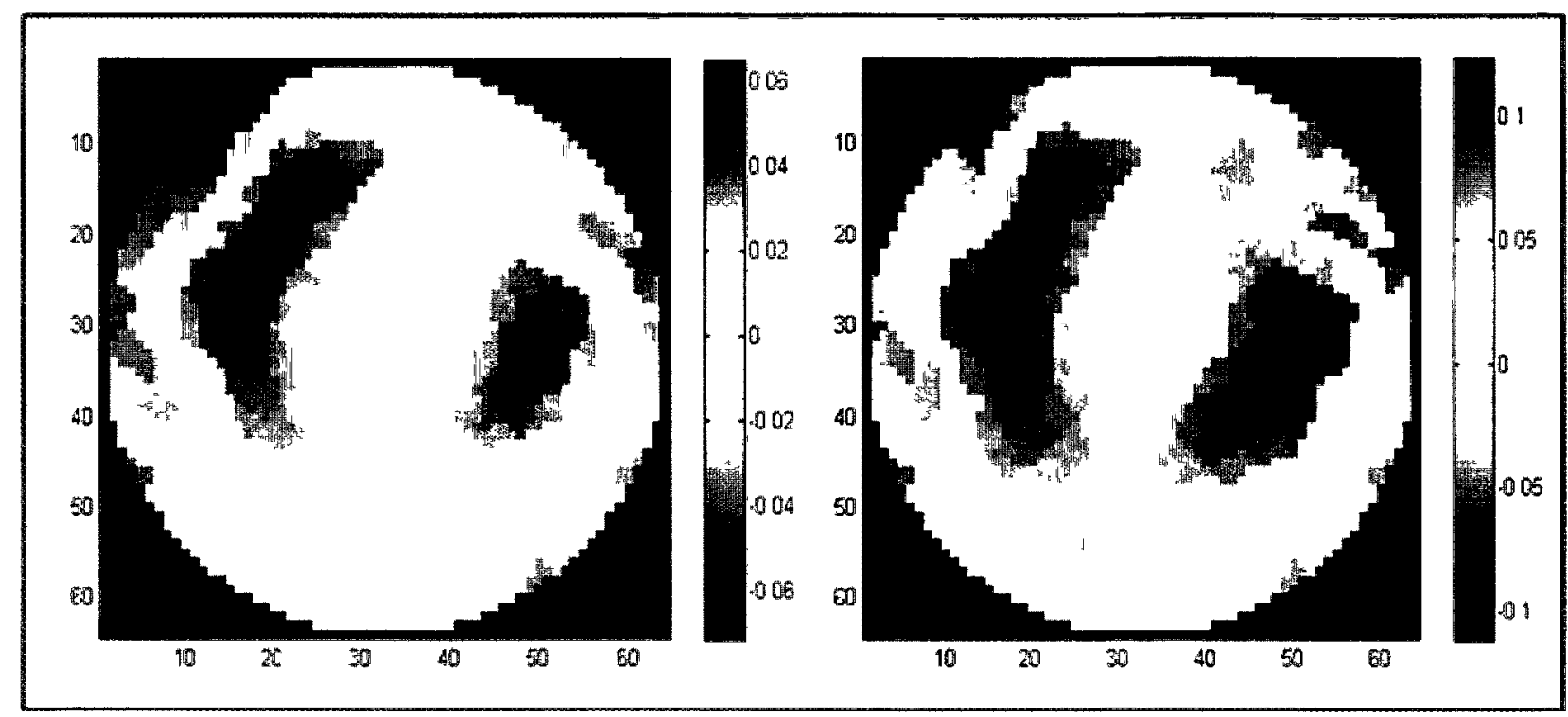

Figure 5- 3: EIT images of a chest cross section of a diseased pig (lung injury due to lavage), (left) lungs before air filling $(\mathrm{Paw}=0 \mathrm{cmH} 2 \mathrm{O})$, (right) lungs after air filling $(\mathrm{Paw}=40 \mathrm{cmH} 2 \mathrm{O})$. The status of air filled image changes based on the chosen difference point ( ).

As seen in figure 5-3, EIT cross section image (left) of the lungs of ALI diseased pig was taken before air filling. The right image in the same figure is a cross sectional image of the lungs for the same ALI diseased pig after a ir filling. Both images changed in value based o $n$ the reference voltage explained before in equation $5-1$. 


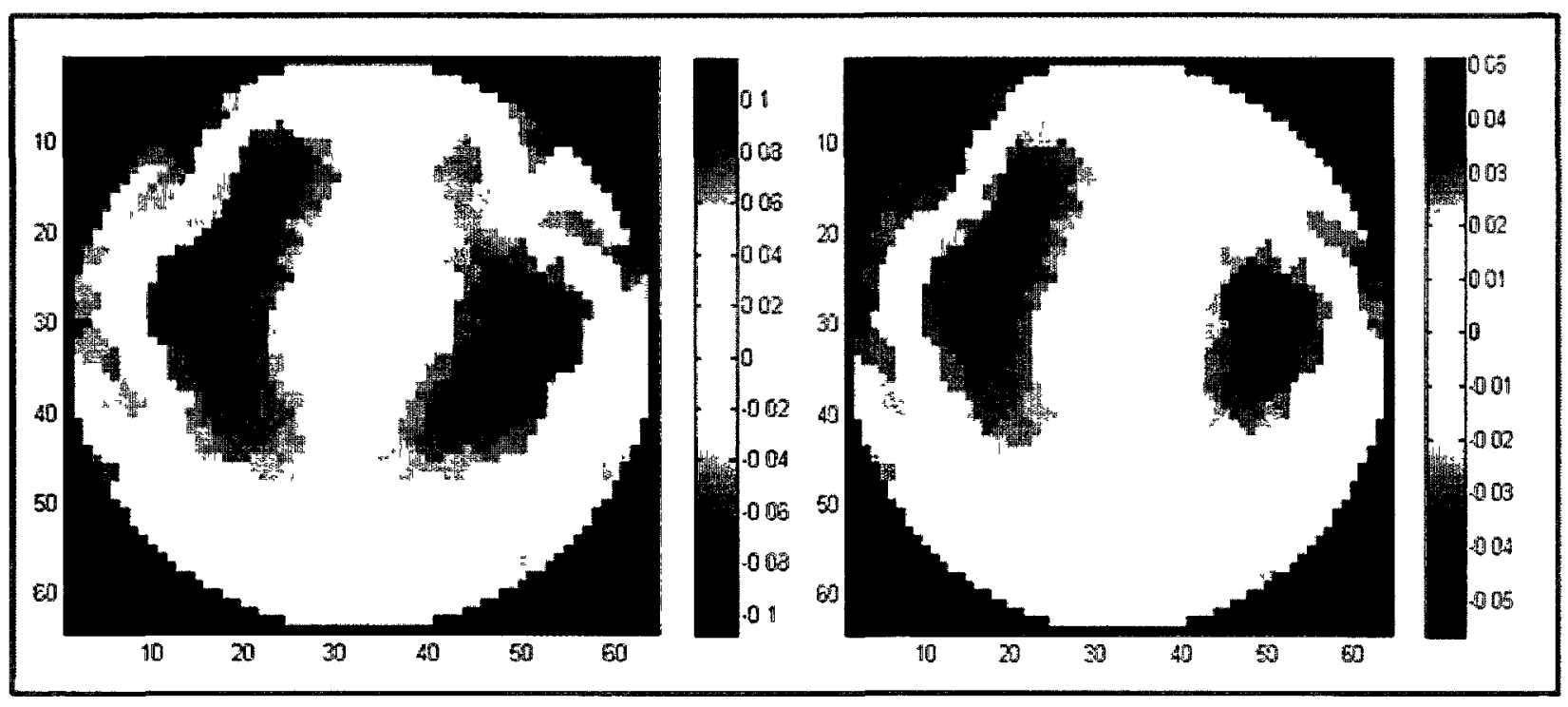

Figure 5- 4: EIT images of a chest cross section of a diseased pig (lung injury due to lavage), (left) lungs before air emptying (Paw $=40 \mathrm{cmH} 2 \mathrm{O})$, (right) lings after air emptying $(\mathrm{Paw}=0 \mathrm{cmH} 2 \mathrm{O})$. The status of air emptied image changes based on the chosen difference point ( ).

As seen in figure 5- 4, for the same ALI diseased pig chosen before, EIT cross sectional image (left) of the lungs was obtained before air emptying. The right image is a cross sectional image of the lungs after the event. Considering the same reference point chosen as before, the values of both images were changed.

EIT data analysis was done through different steps; each is explained in details showing how the research algorithm is implemented to solve such an obstacle in each level. The overall flowchart of the proposed algorithm to analyse EIT data is shown in figure 5- 5. 


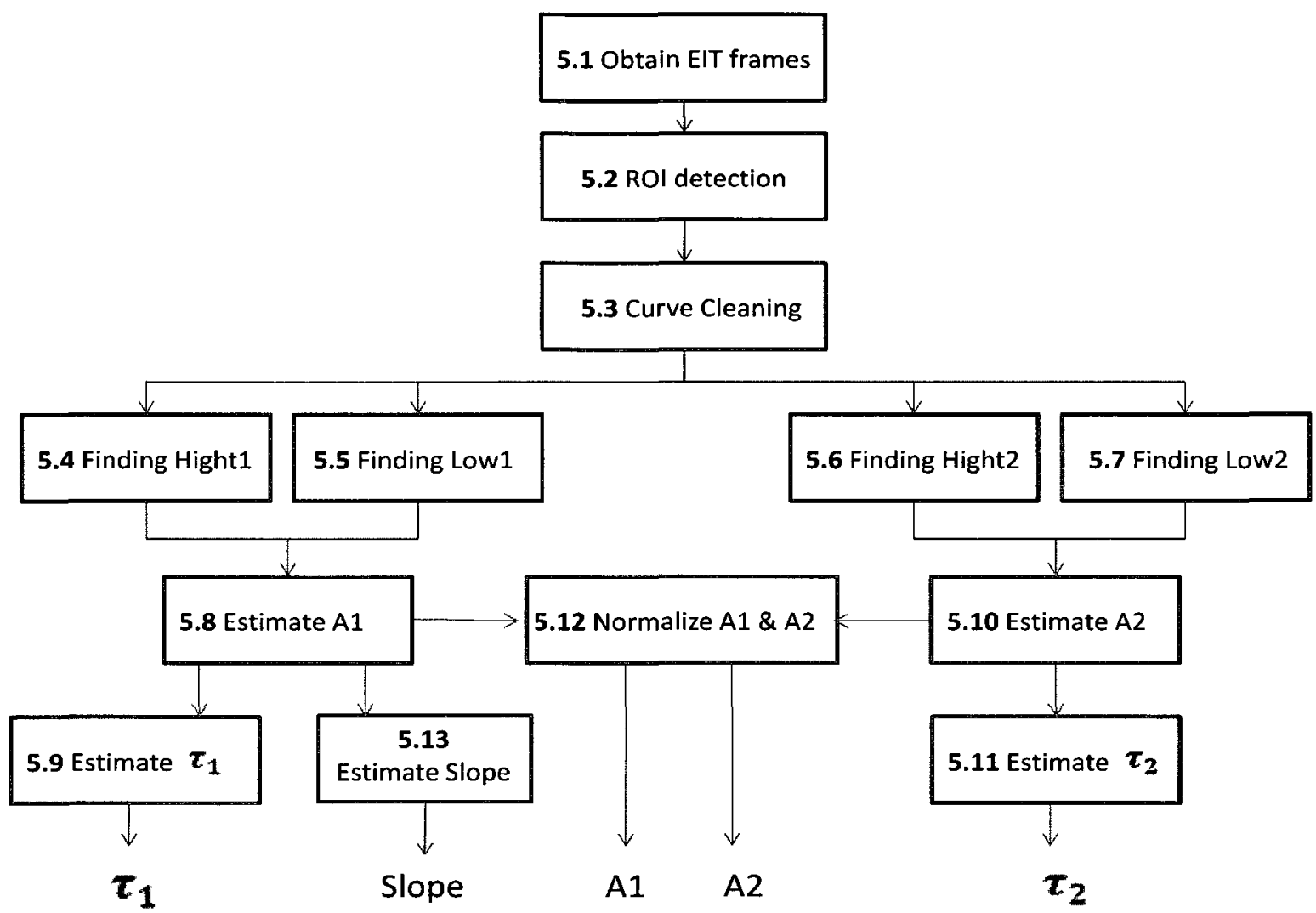

Figure 5- 5: A flowchart describing the algorithm used to analyse EIT data

As seen in figure 5- 5, a flowchart showing the algorithm implemented steps in this thesis project to analyze EIT data. As a result of implementing these steps, research parameters A1 $\tau_{1}$, Slope, A2, and $\tau_{2}$ are estimated for each ROI selected on EIT image of the chosen pig's lungs.

\subsection{Obtain EIT Frames:}

EIT data generated from the EIT s ystem a re p rocessed by utilizing the E IDORS toolkit, open source software suite for image r econstruction in e lectrical i mpedance t omography and 
diffuse optical tomography (Adler and Lionheart, 2006). Using the EIDORS developed methods; an array of EIT frames is obtained for the sample pig data.

\subsection{ROI Detection:}

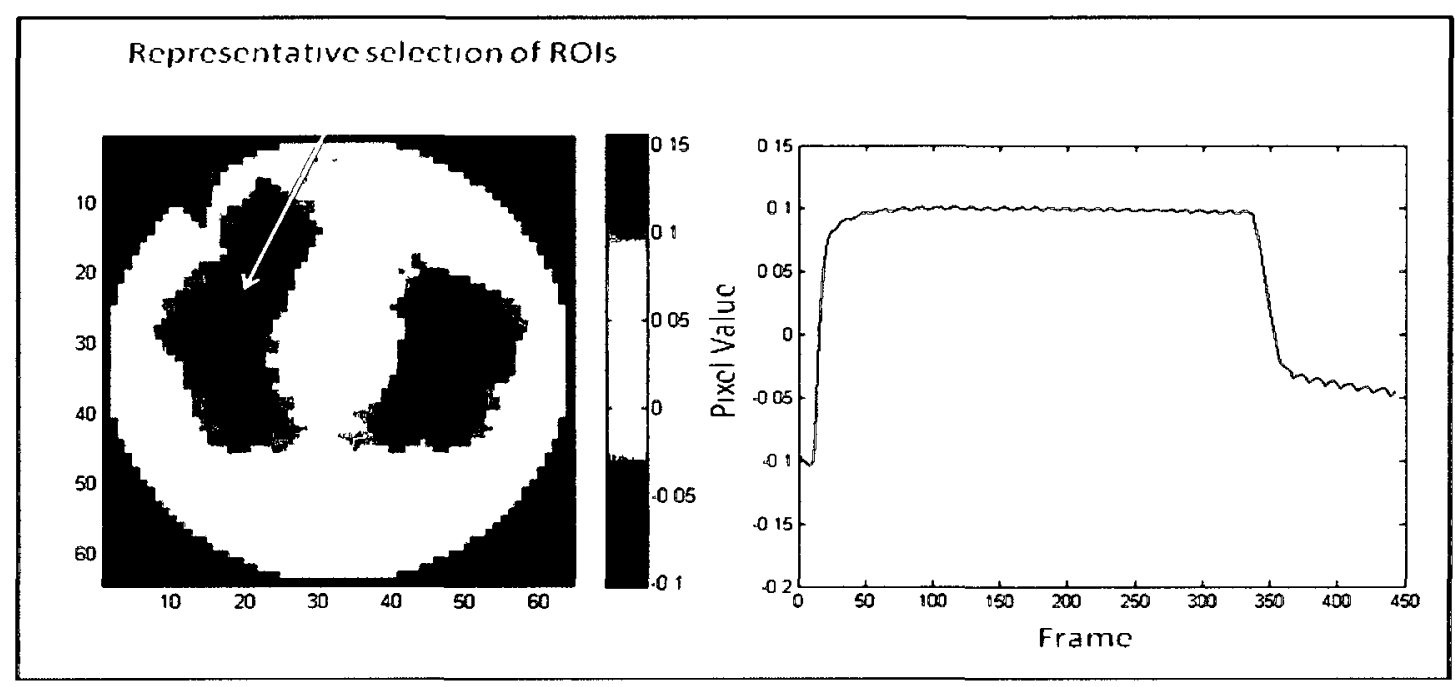

Figure 5- 6: (left) EIT image of the lungs showing a representative selection of regions of interest (ROI). Each blue '*' shows a single ROI in the filled lung image, and (right) a change in pixel value versus frame number as an example of a single ROI graph obtained.

As seen in figure 5-6, left, a representative selection of ROIs are selected on the lungs in the EIT image. It was chosen by the human operator to be away from the heart and the boundaries and in the same region where ROIs selected on the CT images. Each consists of many single ROI. (Right) shows a single ROI versus frame number. Each ROI represents one pixel value on the EIT image and obtained through the whole EIT frames. 


\subsection{Curve Cleaning:}

A curve fitting is applied to each EIT graph obtained from each ROI. This step is needed to eliminate edge sensitivity or any noise that may affect the estimation of such a parameter.

This step finds the coefficients of a polynomial function $P(X)$ of degree $N($ e.g., $N=15$ ) that fits the EIT data curve best in a least-squares sense using MATLAB polyfit function. Then, the coefficients were evaluated to best fit the EIT data obtained at all points of a vector X equal to the length of the EIT data curve using MATLAB polyval function.

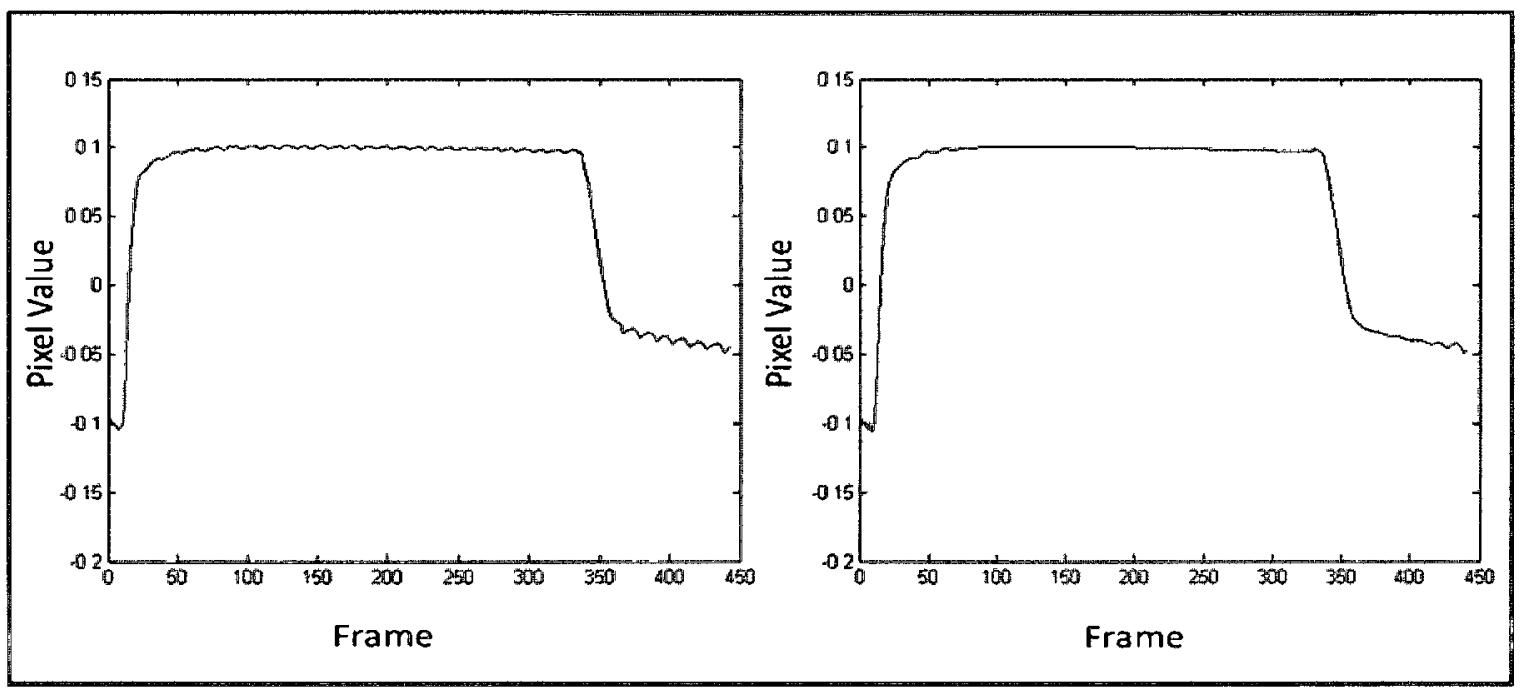

Figure 5- 7: (left), a ROI pixel value versus frame number curve of an EIT image of the lungs before applying curve fitting step, (right) the same curve after applying curve fitting step.

As seen in figure 5- 7 (left), pixel value versus frame number curve represents a selected ROI on EIT image. The curve fitting step is not applied here. Figure 5- 7 (right), shows the same curve for the same ROI selected on the EIT image but after applying the curve fitting step which is needed to get rid of the curve edges noise. 


\subsection{Finding High1:}

This step is to find the highest point on the EIT curve at the end of air filling. The highest point is found by iterating through the first half of the curve and finding the maximum point at the end of air filling (figure 5-8).

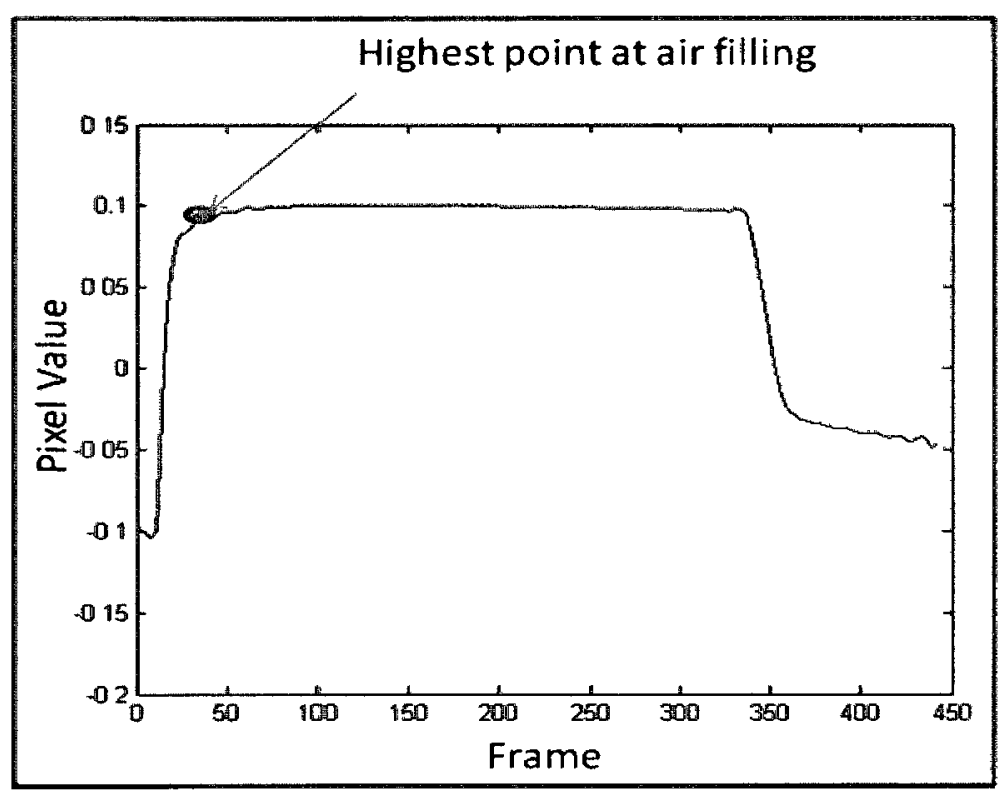

Figure 5- 8: Pixel value of a selected single ROI versus frame number curve of an EIT image of the lungs, it shows a blue circle indicating where the highest point found on the curve during air filling.

As seen in figure 5-8, a pixel value versus frame number curve for a selected ROI on the EIT image showing the "high1" point. The blue circle is placed on the highest point found on the curve at the end of air filling. The estimated highest point found is obtained for all the ROI and will be used in the next analysis steps. 


\subsection{Finding Low1:}

This step is to find the lowest point on the curve that represents the start of 1 ung a ir filling. The lowest point is found by iterating and finding the minimum point through the first half of the curve and before "high1" found before (figure 5-9).

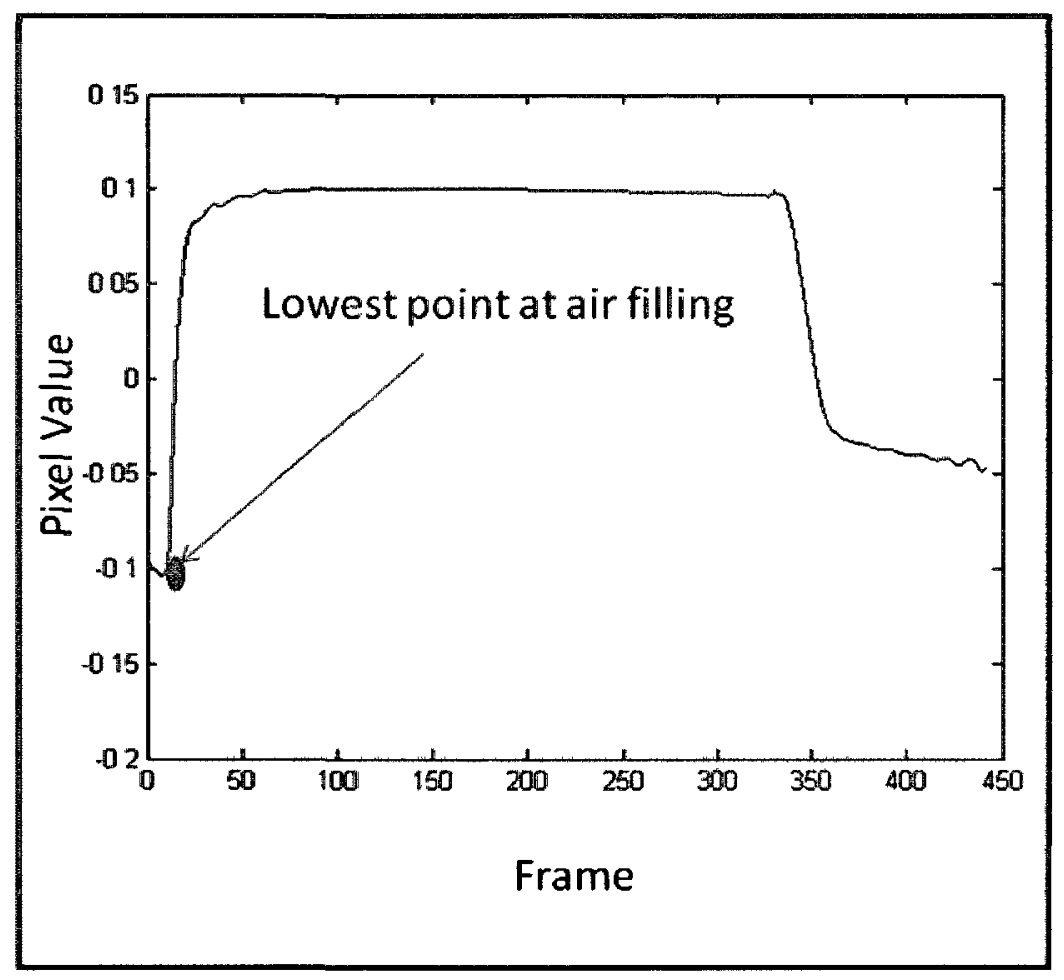

Figure 5- 9: Density of a selected single ROI versus frame number curve of an EIT image of the lungs, it shows a blue circle indicating where the lowest point found on the curve during air filling.

As seen in figure 5-9, a pixel value versus frame number curve for a selected ROI on the EIT image showing the "low1" point. The blue circle is placed on the lowest point found on the 
curve at the start of air filling. The estimated lowest point found was obtained for all the ROI and will be used in the next analysis steps.

\subsection{Finding High2:}

This step is to find the highest point on the curve that represents the start of 1 ung a ir emptying. The highest point is found $\mathrm{b} y$ iterating through the $\mathrm{s}$ econd $\mathrm{h}$ alf $\mathrm{o} f \mathrm{t}$ he curve and finding the maximum point before the curve drops down (lungs air emptying) (figure 5-10).

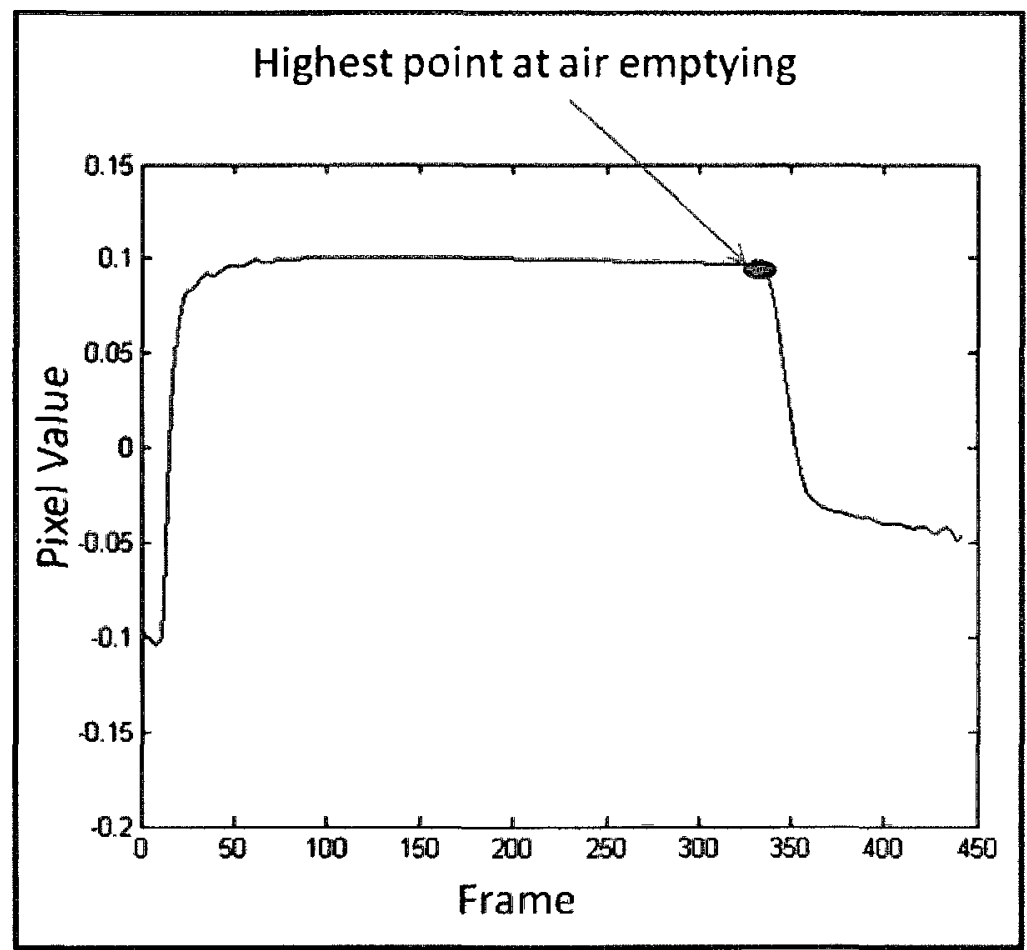

Figure 5- 10: Pixel value of a selected single ROI versus frame number curve of an EIT image of the lungs, it shows a blue circle indicating where the highest point found on the curve during air emptying. 
As seen in figure 5-10, pixel values versus frame number curve for a selected ROI on EIT image showing high2 point. The blue circle indicates where the highest point found on the curve at the start of air emptying and before the curve drops down. The estimated highest point found is obtained for all the ROI and will be used in the next analysis steps.

\subsection{Finding Low2:}

This step is to find the lowest point on the curve that r epresents the end of 1 ung air emptying. The lowest point is found by iterating and finding the minimum point after "high2" where the curve drops down and at the end of air emptying (figure 5-11).

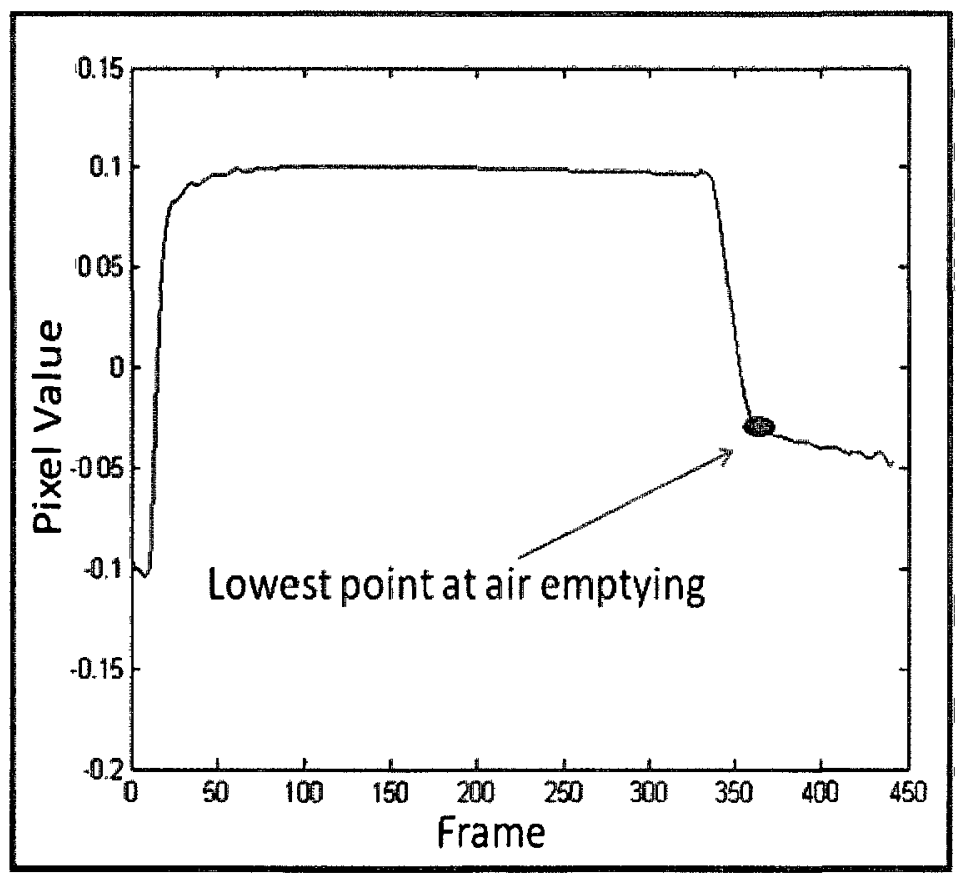

Figure 5- 11: Pixel value of a selected single ROI versus frame number curve of an EIT image of the lungs, it shows a blue circle indicating where the lowest point found on the curve during air emptying.

As seen in figure 5- 11, a pixel value versus frame number curve for a selected ROI on EIT image showing low2 point on the curve. The blue circle indicates where the lowest point 
found on the curve at the end of air emptying. The estimated lowest point found is obtained for all the ROI and will be used in the next analysis steps.

\subsection{Estimating A1:}

This step is to estimate research parameter A1 on the pixel value versus frame number curve. To avoid edge curve sensitivity at the start and end of air filling on the curve (figure 512), the process of this step uses the "high1" and "lowl" points to find new highest and lowest points. A1 is estimated by iterating through the EIT c urve o btained to find new highest and lowest points which are new values of high1 and low1 after eliminating $20 \%$ of each.

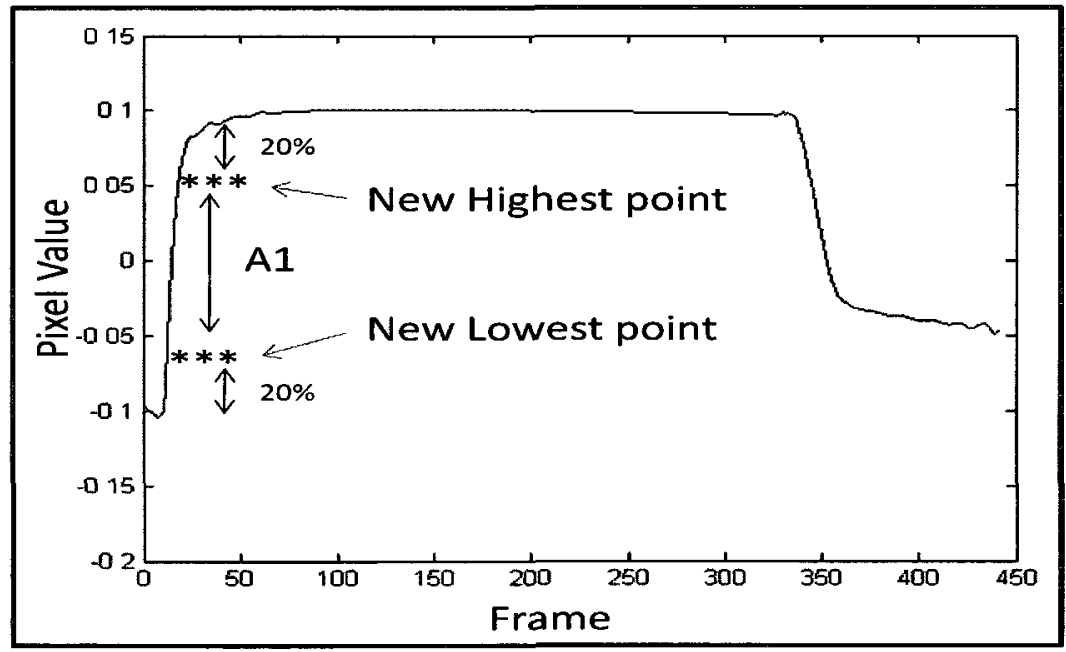

Figure 5- 12: pixel value of a selected single ROI versus frame number curve of an EIT image of the lungs, it shows the new highest and lowest points "***" found after eliminating $20 \%$ of the original highest and lowest points found before. A1 is estimated from the new highest and lowest points during air filling.

As seen in figure 5-12, a pixel value versus frame number curve for a selected ROI on EIT image showing the estimated A1 research parameter. New highest and lowest points a re detected on the curve after eliminating $20 \%$ of high 1 and low1 points. 


\subsection{Estimating :}

This step is to estimate research parameter $\tau_{1}$ on the EIT curve obtained. The process of this step uses the new highest and lowest points detected on the curve and obtained in steps 5.8, Estimating A1.

Research parameter $\tau_{1}$ is estimated by finding the indexes of the new highest and lowest points on the curve (figure 5-13). Then, subtracting the index of the new lowest point from the index of the new highest point and the result is converted into seconds by dividing it by 13 (EIT image frequency rate is $13 \mathrm{~Hz}$ ).

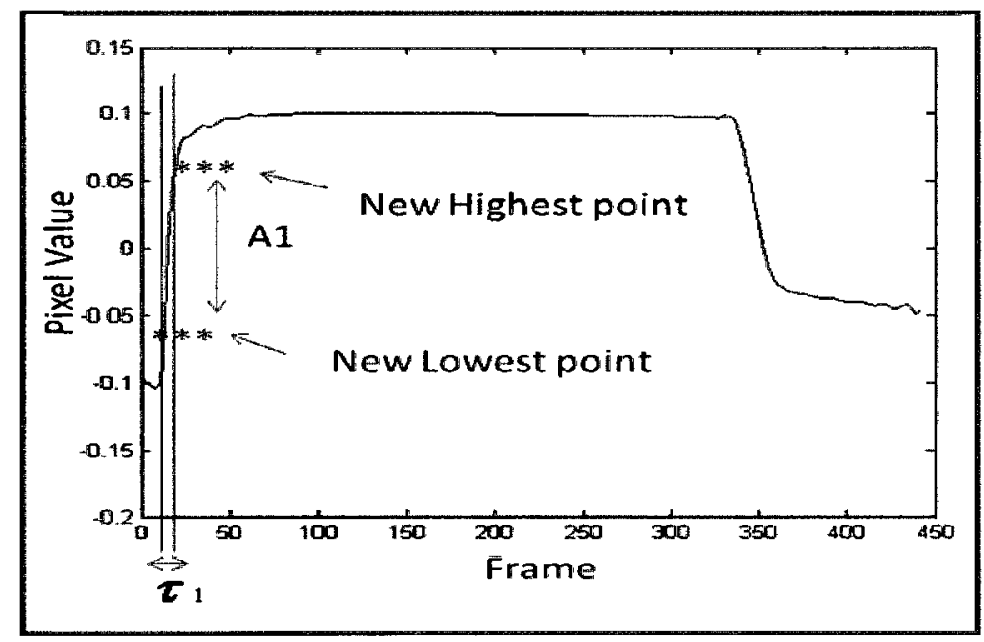

Figure 5- 13: pixel value of a selected single ROI versus frame number curve of an EIT image of the lungs, it shows the estimated research parameter $\tau_{1}$ for air filling.

As seen in figure 5-13, a pixel value versus frame number curve for a selected ROI on EIT image showing $\tau_{1}$ research parameter. The indexes of new highest and lowest points on the curve are used to estimate research parameter $\tau_{1}$ which is measured in seconds. 


\subsection{Estimating A2:}

This step is to estimate the research parameter A2 on the curve for lung air emptying. The process of this step uses high2 and low2 points found in steps 5.6, finding the high2, and 5.7, finding the low2.

A2 is estimated by iterating through the EIT curve to find new highest and lowest points which are new values of high 1 and low1 after eliminating $20 \%$ of each. This process is applied to avoid edge curve sensitivity at the start and end of air emptying on the curve (figure 5-14).

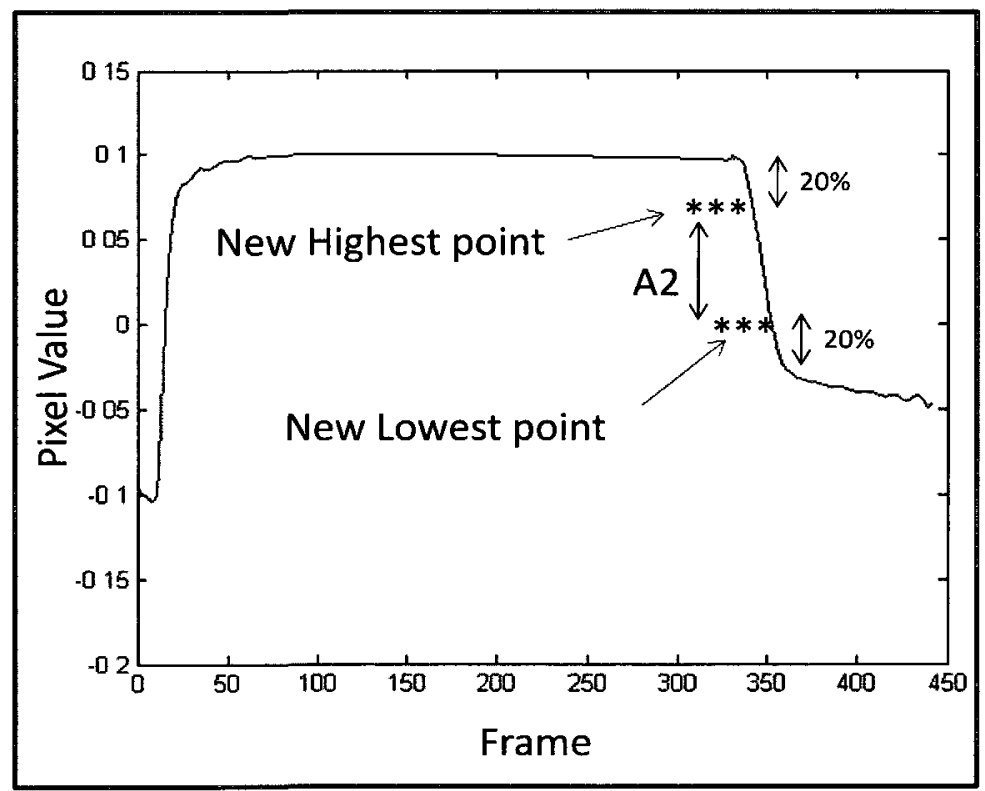

Figure 5- 14: pixel value of a selected single ROI versus frame number curve of an EIT image of the lungs, it shows the new highest and lowest points "***" after eliminating $20 \%$ of high2 and low 2 points. Research parameter A1 is estimated from the new highest and lowest points for air emptying.

As seen in figure 5-14, a pixel value versus frame number curve for a selected ROI on EIT image showing research parameter A2. The new highest and lowest points are detected on the curve after eliminating $20 \%$ of high 2 and low 2 values. 


\subsection{Estimating :}

This step is to estimate research parameter $\tau_{2}$ on the EIT curve obtained. The process of this step uses the new highest and lowest points found on air emptying obtained in steps 5.10.

Research parameter $\tau_{2}$ is estimated by finding the indexes of the new highest and lowest points on the curve (figure 5-15). Then, subtracting the index of the new highest point from the index of the new lowest point and the result is converted into seconds by dividing it by 13 (EIT image frequency rate is $13 \mathrm{~Hz}$ ).

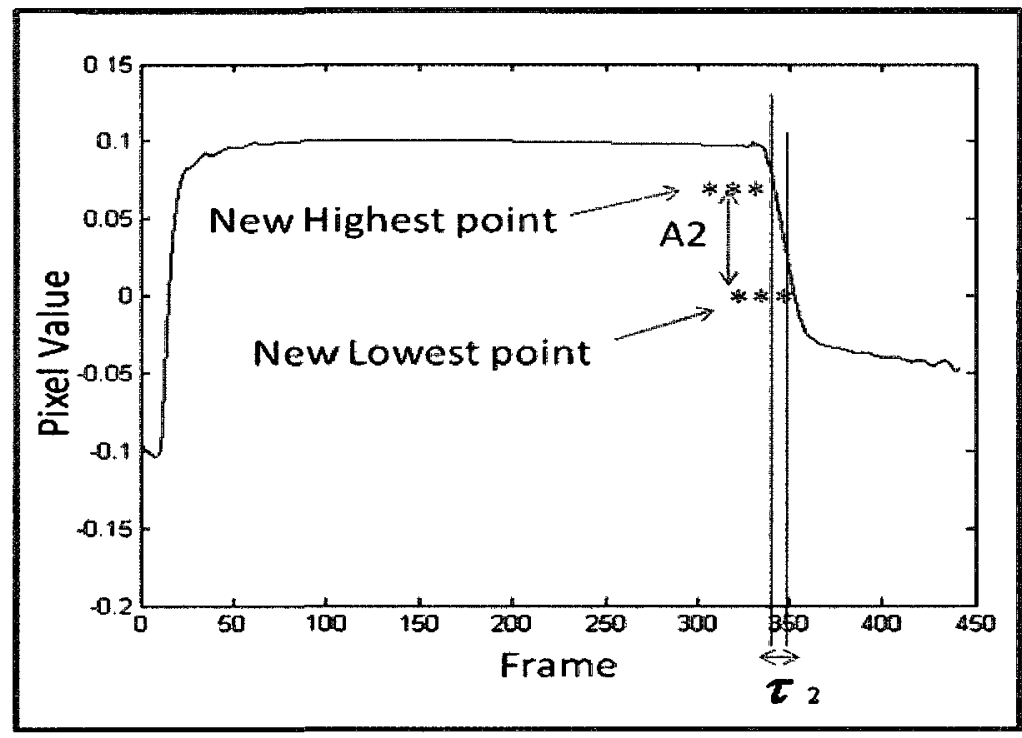

Figure 5- 15: pixel value of a selected single ROI versus frame number curve of an EIT image of the lungs, it shows the estimated research parameter $\tau_{2}$ for air emptying.

As seen in figure 5- 15, research parameter $\tau_{2}$ is estimated on a selected ROI's curve obtained. The indexes of the new highest and lowest points are considered in this estimation. $\tau_{2}$ is estimated in seconds and the process is repeated for all ROIs selected on the image.

\subsection{Normalizing A1 and A2:}


Research parameters $A 1$ and $A 2 \mathrm{w}$ ere $\mathrm{n}$ ormalized $\mathrm{b}$ y $\mathrm{f}$ inding $\mathrm{t}$ he $\mathrm{m}$ ean $\mathrm{v}$ alue $\mathrm{o} f \mathrm{e}$ ach parameter set and then dividing each single estimated parameter by the obtained mean value.

This step is needed because research parameters A 1 and A2 estimated on the EIT image's ROIs vary from one to another. For example, A1 e stimated on a $\mathrm{g}$ raph o btained from ROI selected on the top of the lungs is higher than the same parameter estimated on ROI selected on the middle or on the bottom of the lungs.

\subsection{Estimating the Slope:}

This step is to estimate the slope of the curve located between air filling and emptying. It fits the best straight line on the curve between end of a ir filling and beginning of a ir e mptying at degree $\mathrm{N}=1$ (figure 5-16).

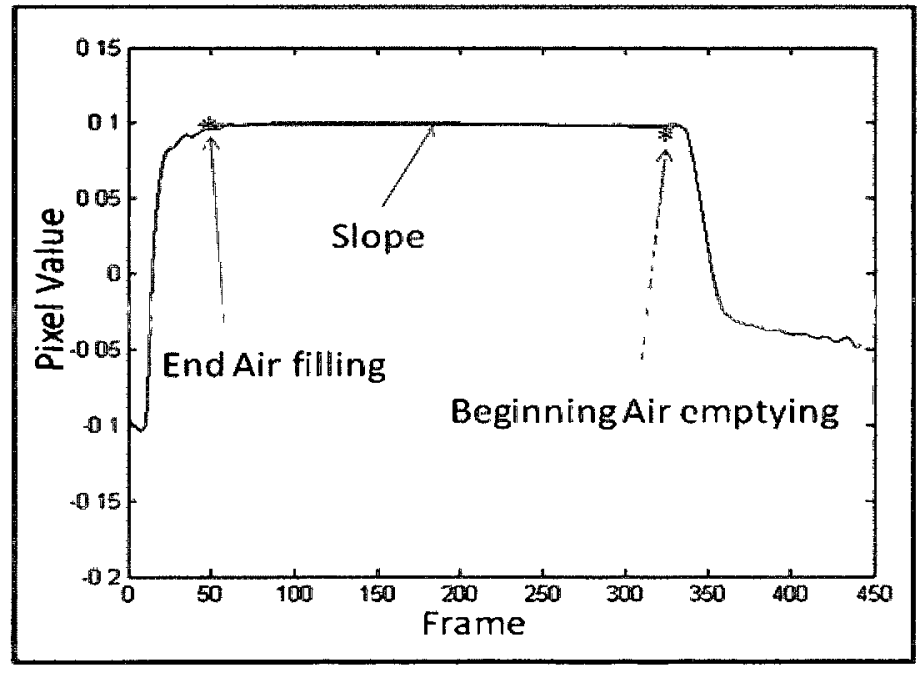

Figure 5- 16: pixel value versus frame number curve of a ROI selected on an EIT image of the lungs. Red line on the curve shows the slope between end of air filling and beginning of air emptying. 
As seen in figure 5-16, the slope is found and shown on the graph between air filling and emptying. A straight line is best fitted into the curve. The slope of the straight line is found and estimated for all ROI pixel value versus frame number curves.

The slope is normalized to research parameter A1 by dividing each ROI's slope obtained by the mean value of research parameter A1 set obtained before. 


\section{Chapter 6}

\section{Results:}

This chapter shows the research results obtained when implementing the analysis algorithm on different data acquired from different pigs. The results are presented in two parts, from a sample healthy pig labelled "ards22_03", and from the same pig after lung lavage (pig is ALI diseased). In each part, the results are obtained from EIT and CT images for the same pig. Different results from different pigs labelled "ards22-4, 5, and 8" are presented as well. The excluded pig's results are presented at the end of this chapter.

\subsection{Healthy Pig Results:}

Research results presented are obtained from a sample pig labeled "ards22-03". Different representative selections of ROIs were chosen by the human operator and selected on left and right lungs, top, middle, and bottom, for both CT and EIT images (please see figure 6-1, 2). 130 ROIs were selected on both images. Each representative selection of ROIs was selected on the same region on the CT and EIT images.

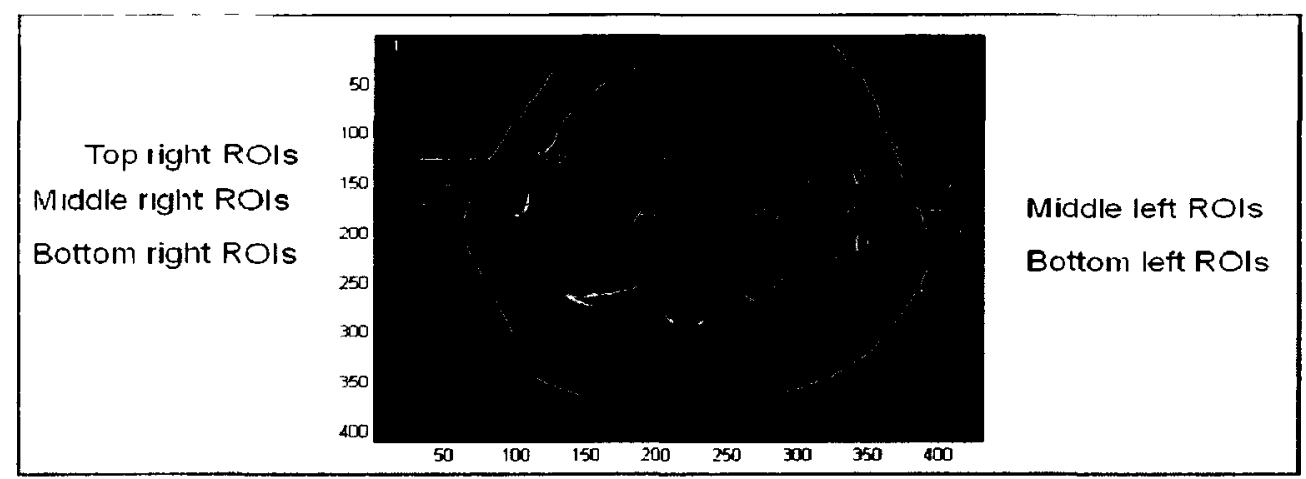

Figure 6- 1: CT cross sectional image of a sample healthy pig labelled "ards22-03" showing the locations of a representative selection of 130 ROIs on the left and right lungs. 
As shown in figure 6- 1, representative selections of 130 ROIs were chosen on CT cross sectional image of the lungs. Different sets of ROIs were selected by the human operator on the top, middle, and bottom of both lungs left and right.

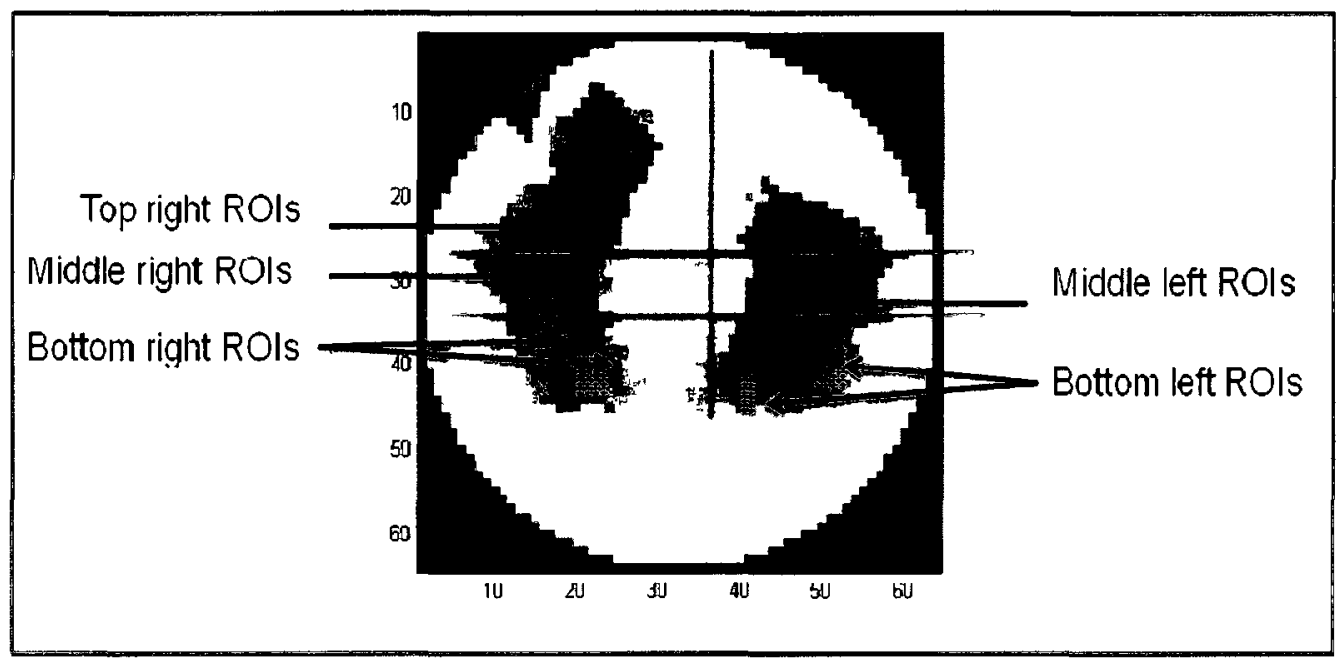

Figure 6- 2: EIT image of the same healthy pig labelled "ards22-03" showing the locations of a representative selection of 130 ROIs on the left and right lungs.

As seen in figure 6- 2, Representative selections of ROIs were chosen on the EIT image of the lungs, selected on the top, middle, and bottom of each lung left and right. Each representative selection of ROIs was chosen by the human operator on the same region on the lungs for both EIT and CT images. The distances (vertical and horizontal) between each representative selection of ROIs and the chest boundaries were considered the same for both EIT and $\mathrm{CT}$ images and chosen by the human operator when selection the region.

Dynamic CT and EIT images for the sample pig were studied and analyzed using the analysis algorithm, described in chapter 4 and 5, through a representative selection of 130 ROIs selected on both lungs. After analyzing all CT image ROIs, each was represented in a density 
versus frame number graph. Also, pixel's value versus frame number graph representing each ROI on the EIT image was obtained. 5 research parameters, explained in chapter 4, were estimated from both graphs for each ROI. For example, considering all the 130 ROIs for both EIT and CT images, ROI number 40 (as shown in figure 6-3) shows close estimation for the 5 research parameters.

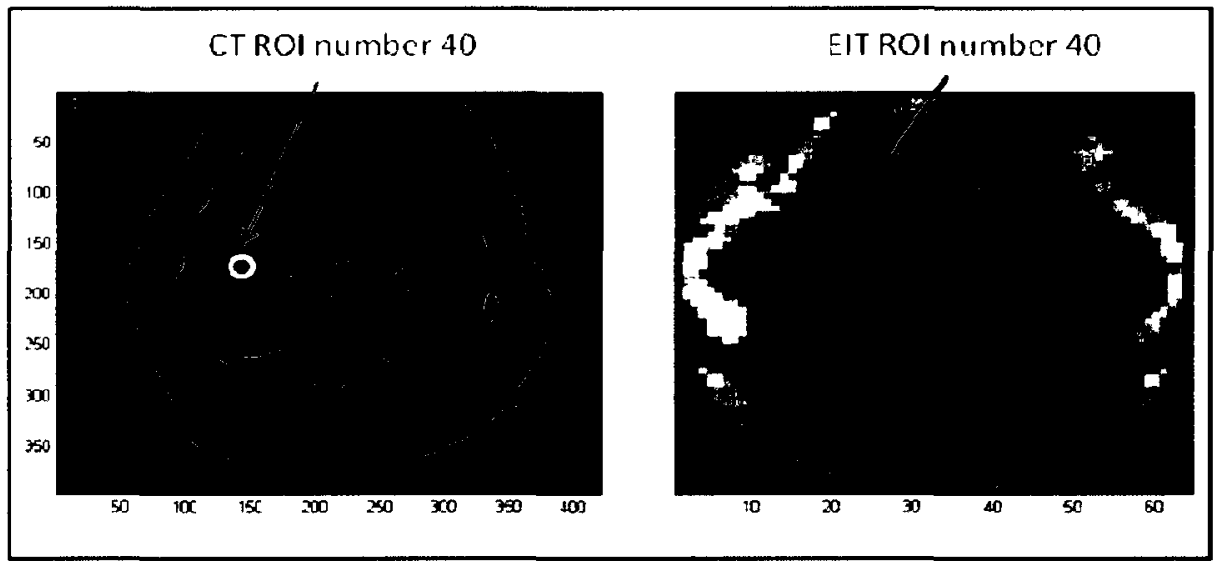

Figure 6- 3: (left) CT image of the sample healthy pig labelled "ards22-03" showing the location of a selected ROI number 40 on the right lung, (right) EIT image for the same healthy pig showing the same location of the selected ROI number 40.

As shown in figure 6-3, ROI number 40 of the representative selections 130 ROIs, located in the right lung and chosen on both CT image (left) and EIT image (right). For example, the ROI number 40, on both CT and EIT images, match each other based on estimating the 5 research parameters (please see figure 6-4). 

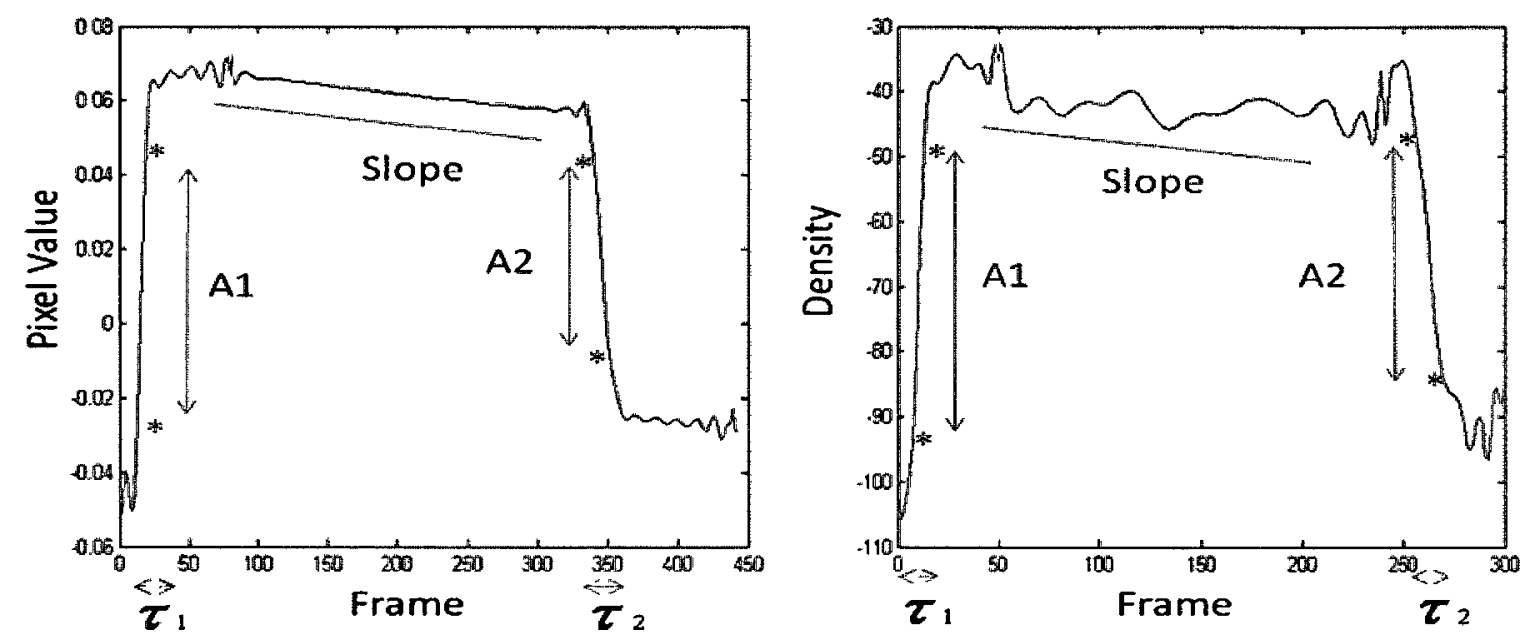

Figure 6- 4: (left) the chosen ROI number 40 pixel value versus frame number graph for EIT image of the sample healthy pig labelled "ards22-03", (Right) ROI number 40density versus frame number for CT image of the same healthy pig. Both graphs show the 5 research parameters that were estimated.

As shown in figure 6- 4, (left) p ixel value v ersus frame $\mathrm{n}$ umber $\mathrm{g}$ raph represents the chosen ROI number 40 on the EIT image. The right figure shows the density versus the frame number for the chosen ROI on the CT image. The five research parameters estimated on each graph is shown the following table 6- 1.

\begin{tabular}{|c|c|c|c|c|c|}
\hline $\begin{array}{c}\text { Imaging } \\
\text { technique }\end{array}$ & $\mathbf{A 1}(\square)$ & $\tau_{1}$ (second) & Slope & $\mathbf{A 2}(\square)$ & $\tau_{2}$ (second) \\
\hline Dynamic CT & 0.976 & 0.4 & $5.56 \times$ & 1.11 & 1.3 \\
\hline EIT & 0.990 & 0.5 & $5.87 \times$ & 1.04 & 1.1 \\
\hline
\end{tabular}

Table 6- 1: 5 research parameters estimated from both CT and EIT images for selected ROI number 40 of the representative 130 ROIs obtained from healthy pig labelled "ards22-03". 
As seen in table 6- 1, EIT image ROI number 40 selected on the right lung is validated using the 5 research parameters that were estimated from both EIT and CT data obtained.

All 130 ROIs selected on both EIT and CT images were analyzed and parameters were estimated. Each ROI estimated parameters on the EIT image correspond to the selected ROI estimated parameters on the CT image.
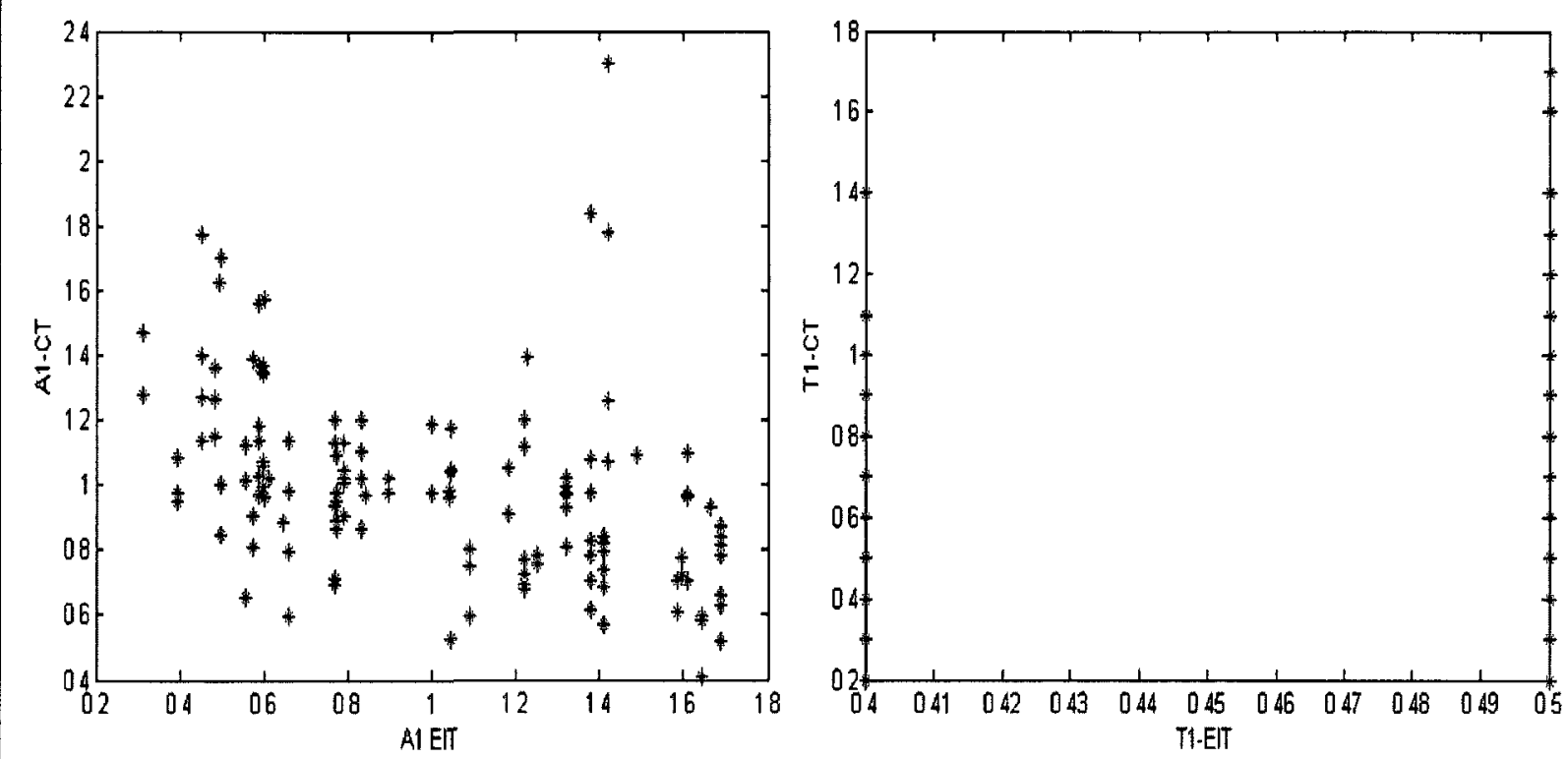

Figure 6- 5: (left), correlation between research parameter Al estimated on 130 ROIs selected on both CT and EIT images obtained from healthy pig labelled "ards22-03", (right) correlation (blue "*" on the sides) between research parameter $\tau_{1}$ estimated on both EIT and CT images of the same pig.

As seen in figure 6- 5 (left), A1 research parameter was estimated on both EIT and CT images. The graph shows negative correlation between A1 estimated on EIT and CT images. The right figure shows poor correlation when comparing the $\tau_{1}$ research parameter estimated on EIT and CT images of the same healthy pig. The estimated $\tau_{1}$ research parameter for EIT data is 
found to be one of the two values 0.4 or 0.5 seconds. This is because of the quantization issues with the time and the low resolution of EIT images.
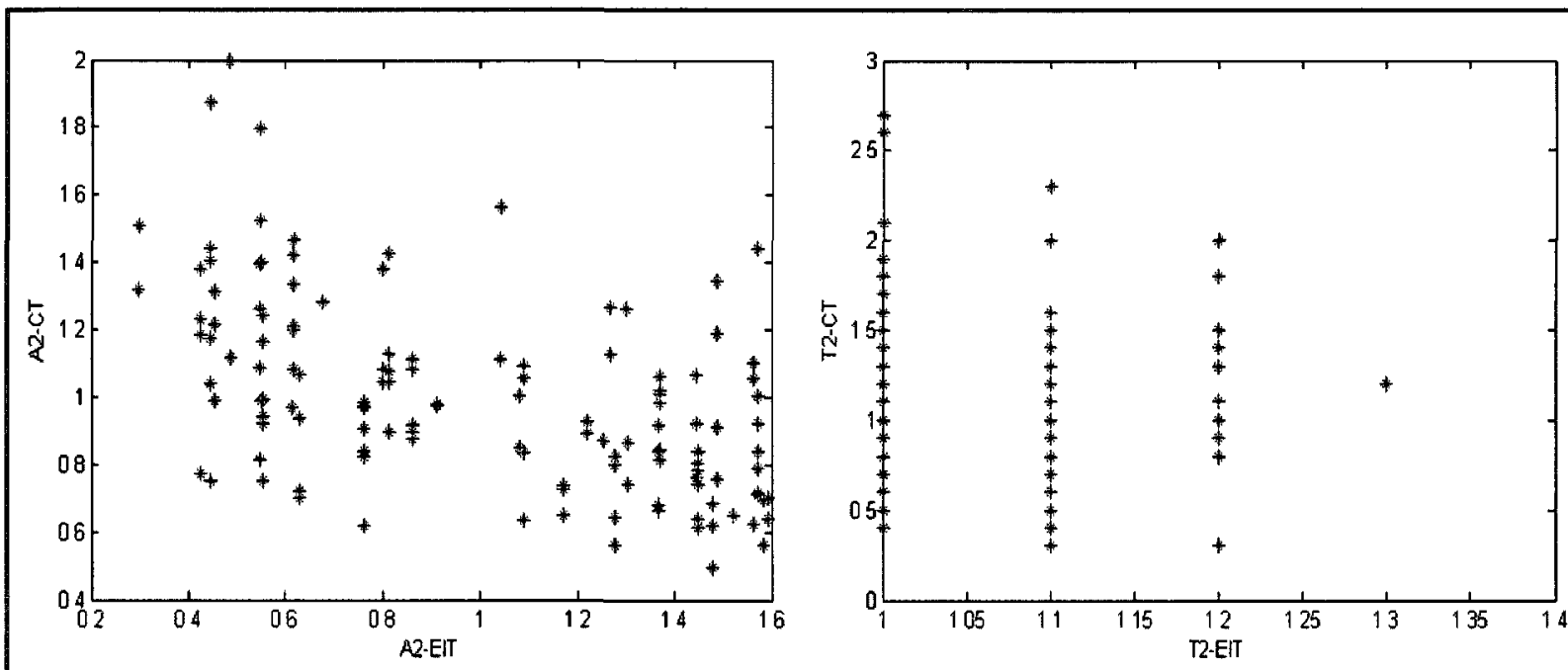

Figure 6- 6: (left), correlation between research parameter A2 estimated on 130 ROIs selected on both CT and EIT images obtained from healthy pig labelled "ards22-03", (right) correlation between research parameter $\tau_{2}$ estimated on both EIT and CT image of the same pig.

As seen in figure 6- 6 (left), A2 research parameter was estimated on ROIs selected on both EIT and CT images. The graph shows a negative correlation between research parameter A2 estimated on ROIs selected on EIT and the same parameter estimated on ROIs selected on CT images. The right figure shows poor correlation for $\tau_{2}$ research parameter estimated on ROIs selected on EIT and CT images. The effect of quantization issues with time is clear in the right figure. The estimated parameter $\tau_{2}$ on the EIT ROIs was found to be 1,1.1,1.2, or 1.3 seconds. There are quantized steps in values for this research parameter estimated on the EIT images. 


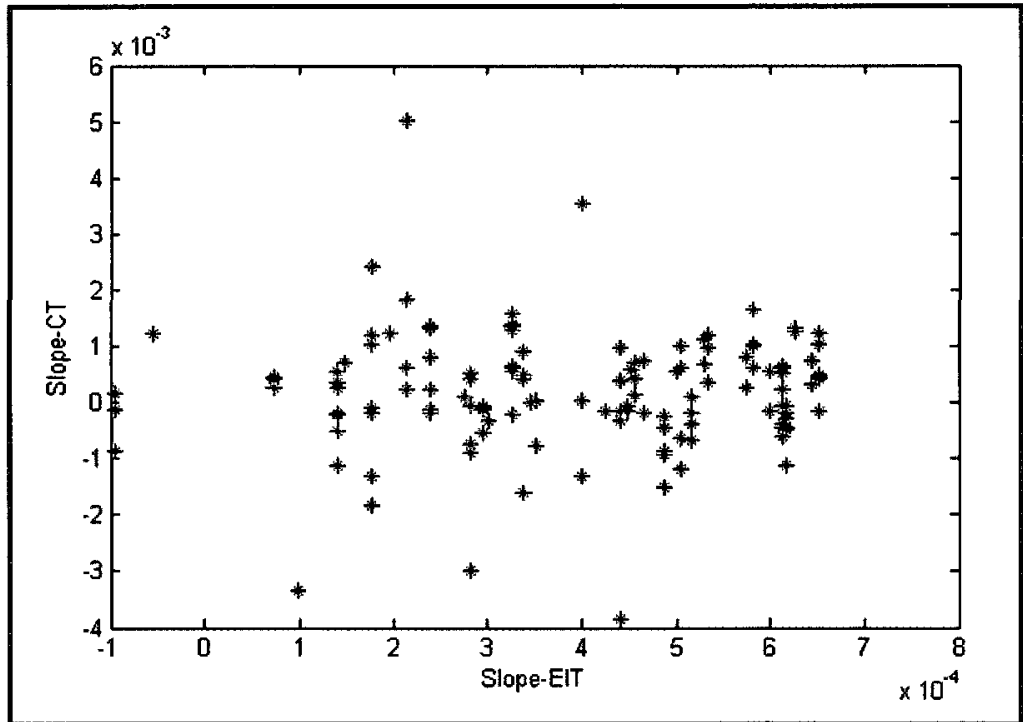

Figure 6- 7: Correlation between research parameter Slope estimated on 130 ROIs selected on both CT and EIT images of healthy pig labelled "ards22-03",

As seen in figure 6- 7, slope research parameter was estimated on ROIs selected on both EIT and CT images. It shows a poor correlation between the slope estimated on EIT and CT ROIs.

\subsection{ALI Diseased Pig Results:}

CT and EIT images for the same pig labeled "ards22-03" were studied and analyzed after lung lavage causing ALI disease. Representative selections of 130 R OIs were chosen on both lungs for both images. The location of the 130 ROIs is the same location chosen for the same pig (healthy) before lung lavage. ROI number 40 with respect to the representative selections, 130 ROIs are chosen as an example of validation between EIT and CT images (please see figure 6-8). 

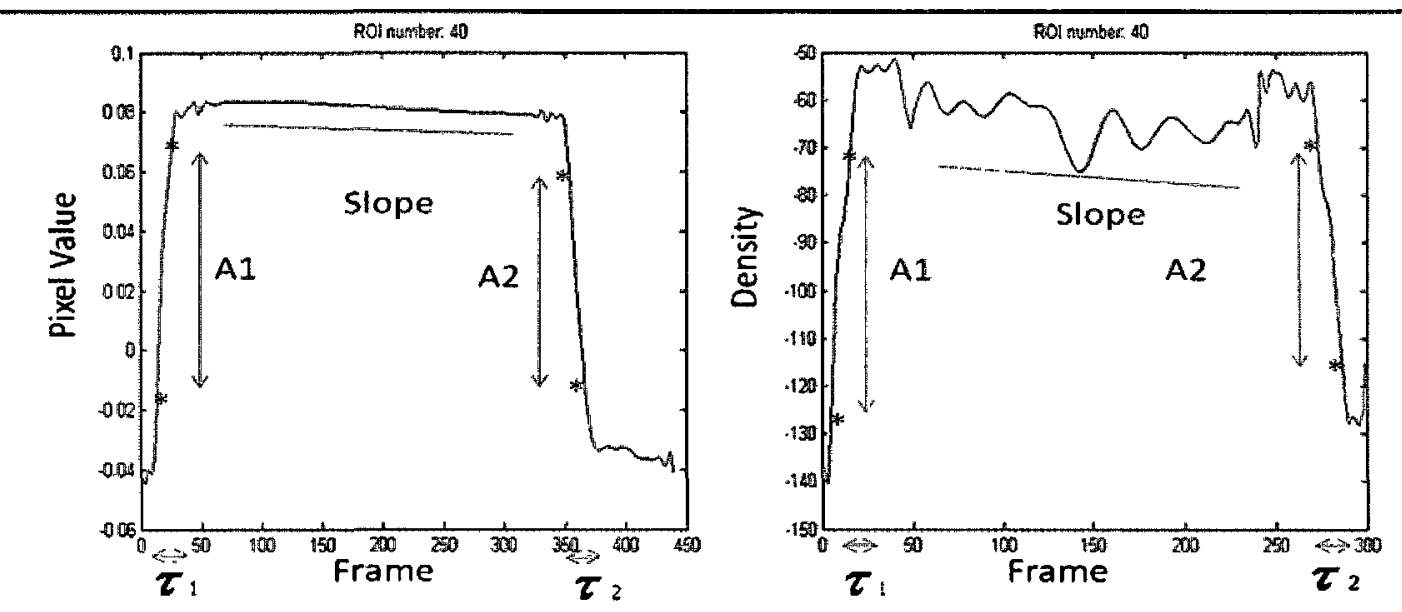

Figure 6- 8: (left) the chosen ROI number 40 pixel value versus frame number graph for EIT image of the lungs of the sample ALI diseased pig labelled "ards22-03", (Right) ROI number 40density versus frame number for CT image of the same healthy pig lungs. Both graphs show the 5 research parameters that were estimated.

As shown in figure 6- 8, (left) pixel value versus frame number graph obtained from EIT image of the lungs for the selected ROI number 40 . The right figure shows the density versus the frame number for the same ROI number 40 on the CT image of the lung for the same pig. The 5 research parameters estimated on each graph is shown the following table 6-2.

\begin{tabular}{|c|c|c|c|c|c|}
\hline $\begin{array}{c}\text { Imaging } \\
\text { technique }\end{array}$ & $\mathbf{A 1}(\square)$ & $\tau_{1}$ (Second) & Slope & $\mathbf{A 2}(\square)$ & $\tau_{2}$ (Second) \\
\hline Dynamic CT & 0.998 & 0.9 & $2.67 \times$ & 1.070 & 1.3 \\
\hline EIT & 1.082 & 0.8 & $2.85 \times$ & 1.101 & 1.3 \\
\hline
\end{tabular}

Table 6- 2: 5 research parameters estimated from both CT and EIT images for selected ROI number 40 of the representative 130 ROIs obtained from ALI diseased pig labelled "ards22-03".

As seen in table 6- 2, ROI number 40 selected on the right lung of EIT image is validated using the 5 research parameters that were estimated from both EIT and C T data obtained. The 
table shows that the five research parameters e stimated values are close for both CT and EIT images.

The chosen 130 ROIs selected on both EIT and CT images of the lungs were analyzed and parameters were estimated. Each ROI selected on the EIT and CT images chosen to be on the same location of the lungs estimated for the same ALI diseased pig.
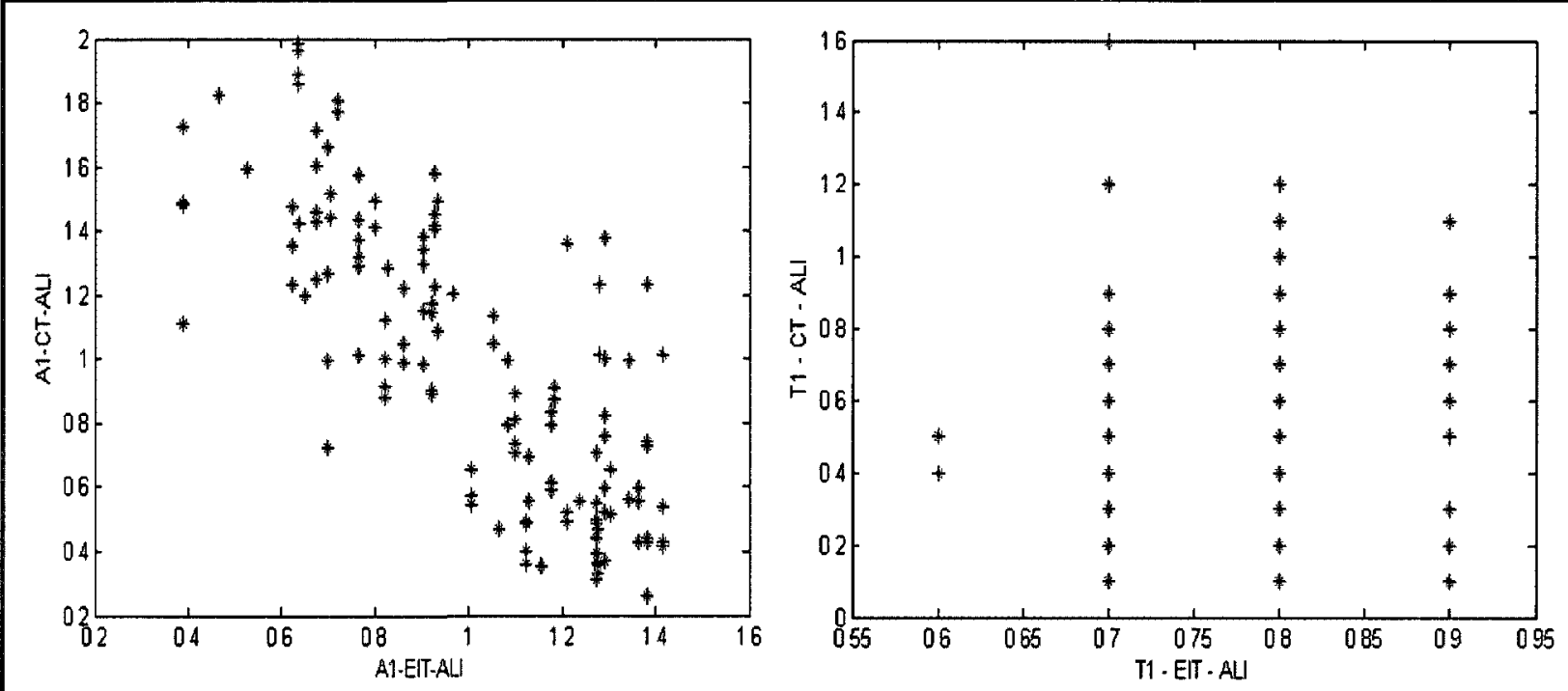

Figure 6- 9: (left), correlation between research parameter Al estimated on 130 ROIs selected on both CT and EIT images obtained from ALI diseased pig labelled "ards22-03", (right) correlation between research parameter $\tau_{\text {। }}$ estimated on both EIT and CT images of the same pig.

As seen in figure 6- 9 (left), Al research parameter was estimated on ROIs selected on both EIT and CT images for the same ALI diseased pig. It shows a negative correlation between A1 estimated on ROIs selected on CT and EIT images. The right figure shows the $\tau_{1}$ research 
parameter estimated on ROIs selected on both EIT and CT images of the same pig. It shows poor correlation between $\tau_{1}$ estimated on EIT and CT ROIs.

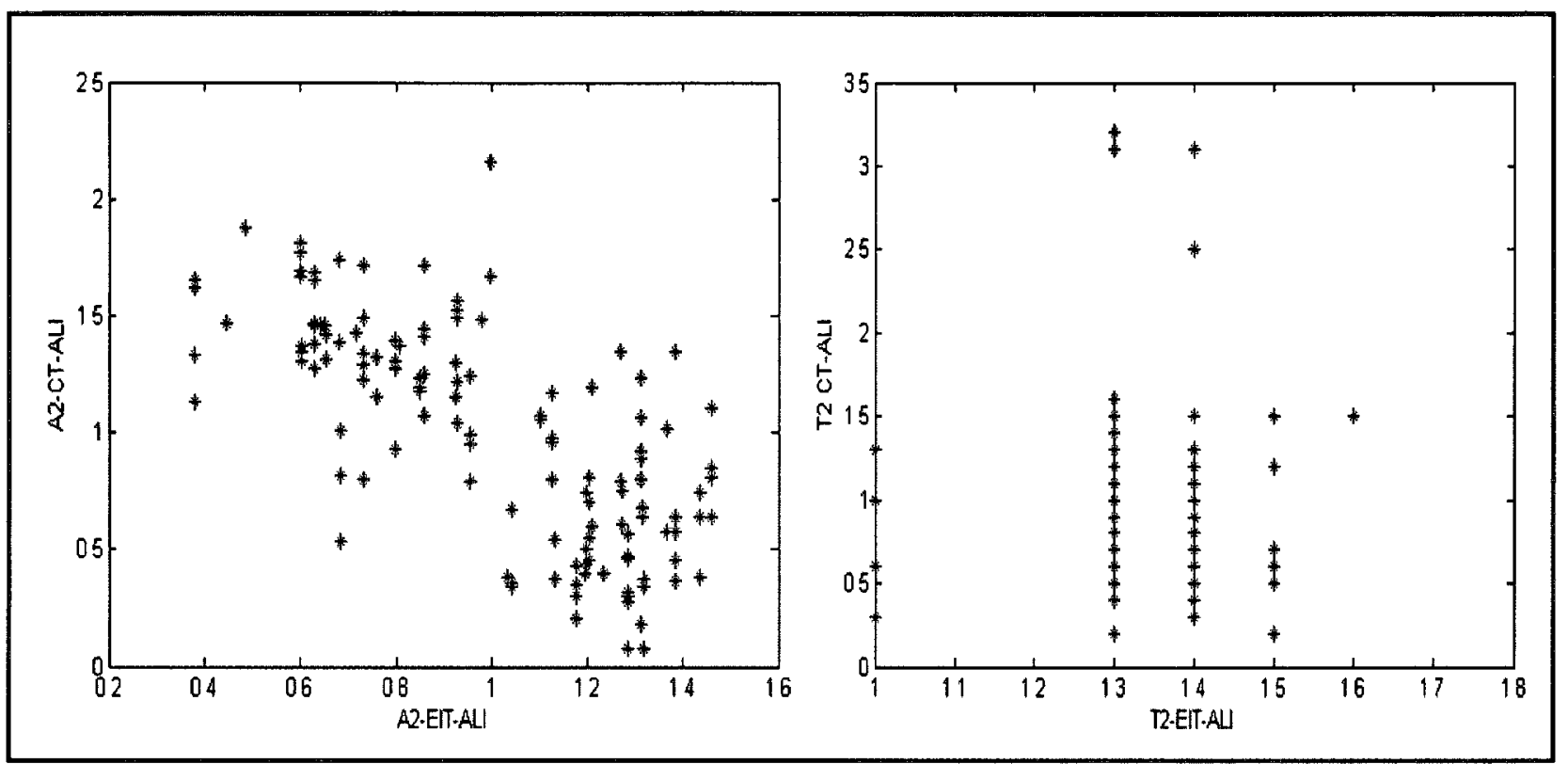

Figure 6- 10: (left), correlation between research parameter A2 estimated on 130 ROIs selected on both CT and EIT images obtained from ALI diseased pig labelled "ards22-03", (right) correlation between research parameter 2 estimated on both EIT and CT image of the same pig.

As seen in figure 6- 10 (left), A2 research parameter was estimated on ROIs selected on both EIT and CT images of ALI diseased $\mathrm{p}$ ig. It $\mathrm{s}$ hows a $\mathrm{n}$ egative $\mathrm{c}$ orrelation $\mathrm{b}$ etween A 2 research parameter estimated on EIT R OIs and C T R OIs. T he $\mathrm{r}$ ight figure $\mathrm{s}$ hows research parameter $\tau_{2}$ estimated on the same ROIs selected on the same images. It shows poor correlation between $\tau_{2}$ estimated on EIT images ROIs and the same parameter estimated on CT images ROIs. 


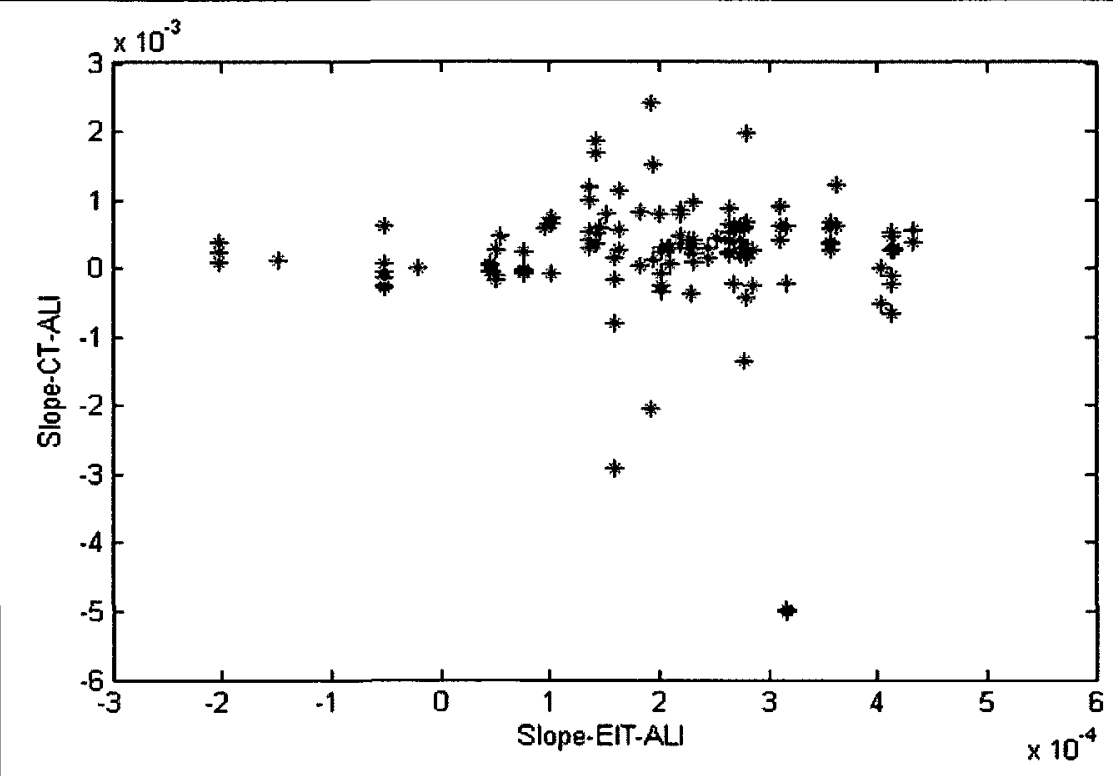

Figure 6-11: Correlation between research parameter Slope estimated on 130 ROIs selected on both CT and EIT images of ALI diseased pig labelled "ards22-03".

As seen in figure 6- 11, slope research parameter was estimated on ROIs selected on both EIT and CT images of the lungs for the same ALI diseased pig. It shows poor correlation between the slope parameter estimated on EIT ROIs and CT ROIs.

5 research parameters were estimated on ROIs s elected on EIT and CT i mages of the other pigs' lungs. Data from 4 pigs labelled 'ards22-03,04, 05, and $08^{\prime}$ were analyzed and studied as shown in table 6-3. 


\begin{tabular}{|c|c|c|c|c|c|c|}
\hline $\begin{array}{c}\text { Pig number } \\
\text { "ards22-" }\end{array}$ & $\begin{array}{c}\text { Healthy/ALI } \\
\text { diseased }\end{array}$ & $\mathbf{A 1}$ & $\boldsymbol{\tau}_{\mathbf{1}}$ & Slope & A2 & $\boldsymbol{\tau}_{\mathbf{2}}$ \\
\hline \multirow{3}{*}{03} & Healthy & -0.424 & 0.142 & 0.005 & -0.515 & 0.021 \\
\cline { 2 - 7 } & ALI diseased & -0.780 & 0.002 & -0.018 & -0.716 & -0.037 \\
\hline \multirow{2}{*}{04} & Healthy & -0.181 & -0.159 & 0.067 & -0.062 & -0.196 \\
\cline { 2 - 7 } & ALI diseased & -0.114 & -0.068 & 0.156 & 0.020 & -0.334 \\
\hline \multirow{2}{*}{05} & Healthy & -0.442 & -0.056 & 0.119 & -0.492 & 0.040 \\
\cline { 2 - 7 } & ALI diseased & 0.045 & 0.047 & 0.123 & 0.132 & 0.044 \\
\hline \multirow{2}{*}{08} & Healthy & -0.575 & -0.033 & 0.163 & 0.010 & -0.125 \\
\cline { 2 - 7 } & ALI diseased & -0.766 & -0.039 & -0.158 & -0.616 & -0.032 \\
\hline
\end{tabular}

Table 6- 3: Correlation coefficients for 5 research parameters estimated on ROIs selected on EIT and CT images of lungs for healthy and ALI diseased pigs labelled "ards22-03, 04, 05, and 08"

As seen in table 6- 3, four pigs' data were analyzed and the 5 research parameters were estimated for each pig data healthy and ALI diseased. The table shows the correlation relationship between each parameter estimated on the CT and EIT data. It shows the correlation coefficients when comparing each parameter estimated on the EIT data to same parameter estimated on the CT data. For example, for pig labelled "ards22-03", there are negative correlations when analyzing research parameters A1 and A2 for both healthy and ALI diseased data.

The five Research parameters were analyzed and studied for each EIT image ROIs and CT image ROIs separately for each healthy and ALI diseased pigs. Each parameter estimated on each ROI is compared to the y-coordinate of the chosen ROIs selected on the EIT image (table 6- 
3). All CT image ROIs estimated parameters were compared to the y-coordinates of each chosen ROI (table 6- 4).

\begin{tabular}{|c|c|c|c|c|c|c|}
\hline $\begin{array}{c}\text { Pig } \\
\text { number } \\
\text { ards22-" }\end{array}$ & $\begin{array}{c}\text { Healthy/ALI } \\
\text { diseased }\end{array}$ & A1 & $\boldsymbol{\tau}_{\mathbf{1}}$ & Slope & A2 & $\boldsymbol{\tau}_{\mathbf{2}}$ \\
\hline \multirow{2}{*}{03} & Healthy & -0.950 & -0.166 & -0.058 & -0.922 & -0.213 \\
\cline { 2 - 7 } & ALI diseased & -0.804 & -0.577 & 0.357 & -0.819 & -0.440 \\
\hline \multirow{2}{*}{04} & Healthy & -0.726 & -0.120 & -0.709 & -0.746 & -0.162 \\
\cline { 2 - 7 } & ALI diseased & -0.653 & -0.297 & -0.220 & -0.639 & 0.351 \\
\hline \multirow{2}{*}{05} & Healthy & -0.824 & 0.200 & 0.882 & -0.913 & 0.024 \\
\cline { 2 - 7 } & ALI diseased & 0.068 & -0.506 & 0.517 & 0.093 & -0.090 \\
\hline \multirow{2}{*}{08} & Healthy & -0.910 & 0.359 & -0.511 & -0.930 & -0.745 \\
\cline { 2 - 7 } & ALI diseased & -0.943 & 0.233 & 0.771 & -0.955 & 0.449 \\
\hline
\end{tabular}

Table 6- 4: Correlation coefficients for the y-coordinate (image row number) of each ROI and 5 research parameters estimated on ROIs selected on EIT images of lungs for healthy and ALI diseased pigs labelled "ards22-03, 04, 05, and 08 "

As seen in table 6- 4, 5 research parameters were compared to the y-coordinate (image row number) of the selected ROIs on the EIT image of the chosen pigs. The table shows the correlation coefficients of each parameter and the y-coordinates of the selected ROIs. For example, for pig labelled "ards22-03", the estimated research parameter A1 negatively correlates with the $y$-coordinate of the selected ROIs on the EIT image. 


\begin{tabular}{|c|c|c|c|c|c|c|}
\hline \multirow{2}{*}{$\begin{array}{c}\text { Pig label } \\
\text { “ards22-" }\end{array}$} & $\begin{array}{c}\text { Healthy/ALI } \\
\text { diseased }\end{array}$ & $\mathbf{A 1}$ & $\boldsymbol{\tau}_{\mathbf{1}}$ & Slope & A2 & $\boldsymbol{\tau}_{\mathbf{2}}$ \\
\hline \multirow{3}{*}{$\mathbf{0 3}$} & Healthy & $\mathbf{0 . 4 6 7}$ & -0.053 & 0.040 & 0.548 & -0.032 \\
\cline { 2 - 7 } & ALI diseased & $\mathbf{0 . 7 9 2}$ & -0.059 & 0.207 & 0.813 & 0.063 \\
\hline \multirow{3}{*}{$\mathbf{0 4}$} & Healthy & $\mathbf{0 . 4 8 0}$ & -0.198 & -0.109 & 0.418 & -0.132 \\
\cline { 2 - 7 } & ALI diseased & $\mathbf{0 . 5 1 6}$ & -0.012 & -0.094 & 0.402 & -0.160 \\
\cline { 2 - 7 } & Healthy & $\mathbf{0 . 4 9 7}$ & -0.132 & 0.020 & 0.448 & -0.204 \\
\hline \multirow{2}{*}{$\mathbf{0 8}$} & ALI diseased & 0.888 & -0.310 & 0.102 & 0.776 & 0.006 \\
\cline { 2 - 7 } & HLI diseased & 0.739 & -0.427 & 0.180 & 0.590 & 0.032 \\
\hline
\end{tabular}

Table 6- 5: Correlation coefficients for the y-coordinate (image row number) of each ROI and 5 research parameters estimated on ROIs selected on CT images of lungs for healthy and ALI diseased pigs labelled "ards22-03, 04, 05, and 08 "

As seen in table 6-5, the estimated 5 research parameters were compared to the $y$ coordinates (image row number) of the chosen ROIs on the CT images. The table shows the correlation coefficients of each parameter in comparison to the y-coordinate of the chosen ROIs. For example, for healthy pig labelled "ards22-03", there is a positive correlation when comparing research parameter A1 to the $\mathrm{y}$-coordinate of the chosen ROIs on the CT image.

Data obtained from both EIT and CT images were processed and analyzed before and after lungs lavage (healthy and ALI diseased pigs images). Research parameters were estimated on both images ROIs for the same healthy and ALI diseased pigs. 

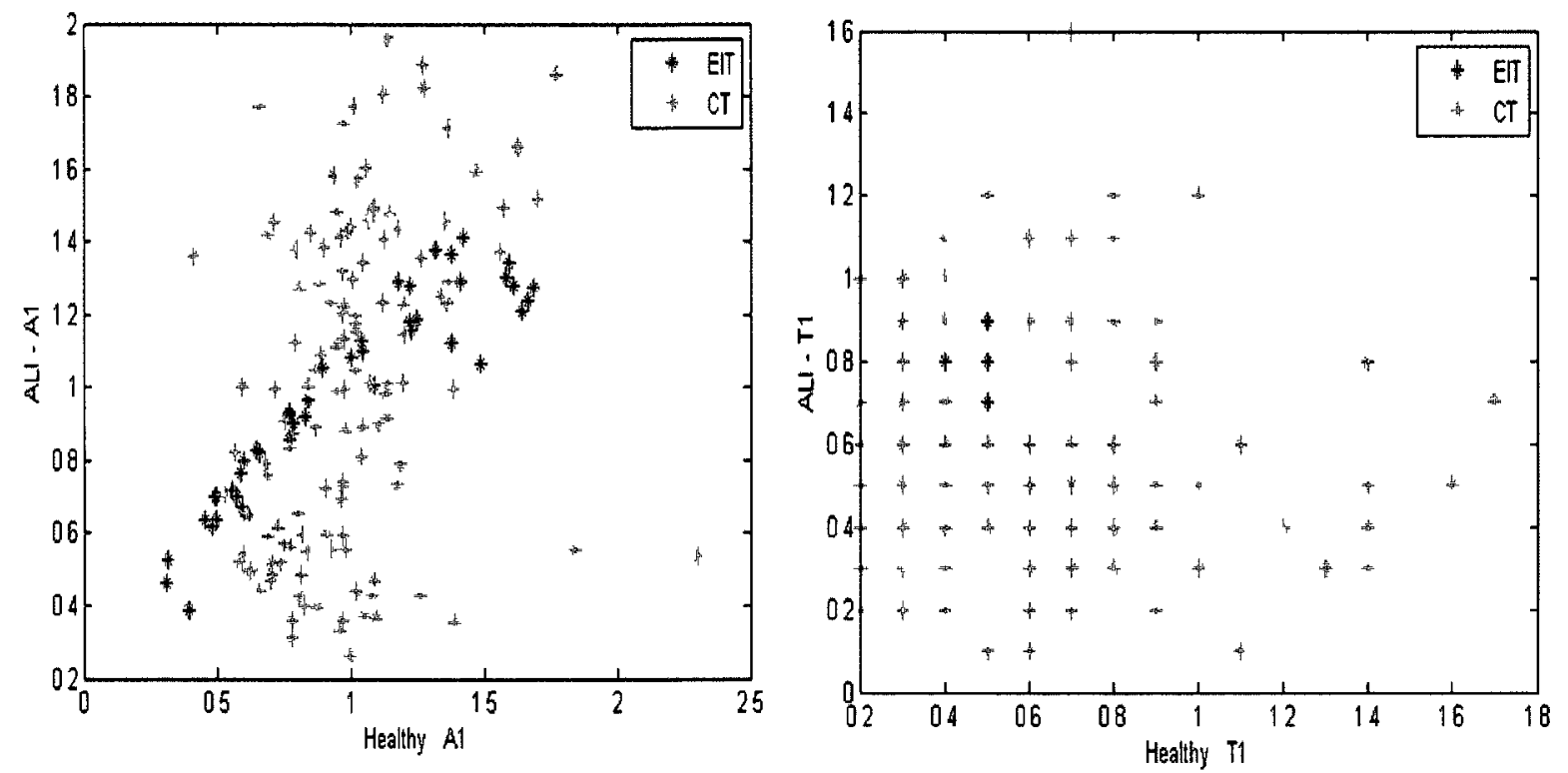

Figure 6-12: (left) A1 research parameter correlation between healthy and ALI diseased pig labelled "ards22-03" for both EIT, blue "*", and CT ,green "*”, image, (right) research parameter correlation for the same healthy and ALI diseased pig EIT , blue “*”, and CT ,green “*”, images.

As seen in figure 6- 12 (left), research parameter A1 was estimated on ROIs selected on both EIT and CT images of both healthy and ALI diseased pig. It shows positive correlation for EIT and CT images ROIs. The right figure shows research parameter estimated on R OIs selected on EIT and CT images for the $\mathrm{s}$ ame $\mathrm{p}$ ig. It $\mathrm{s}$ hows $\mathrm{p}$ oor $\mathrm{c}$ orrelation $\mathrm{b}$ etween $\mathrm{R}$ OIs selected on EIT and CT images of healthy and ALI diseased pig. 


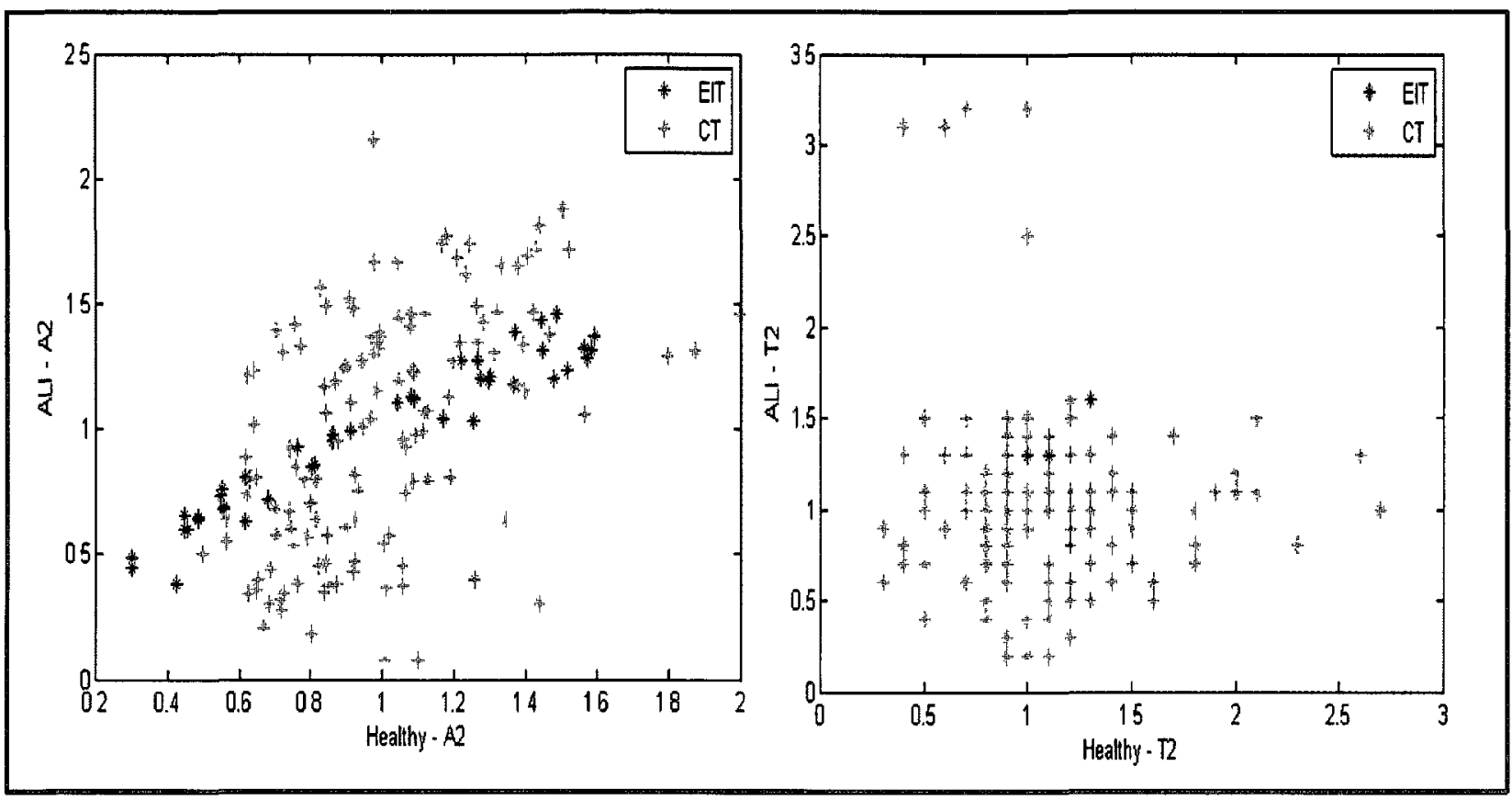

Figure 6- 13: (left) A2 research parameter correlation between healthy and ALI diseased pig labelled "ards22-03" for both EIT , blue "*", and CT ,green "*”, image, (right) research parameter correlation for the same healthy and ALI diseased pig EIT , blue "*”, and CT ,green “*”, images.

As seen in figure 6- 13 (left), A2 research parameter was estimated on ROIs selected on both EIT and CT images for healthy and ALI diseased pig. It shows positive correlation between A2 research parameter estimated on healthy and ALI diseased pig EIT and CT image. The right figure shows the research parameter estimated on ROIs selected on EIT and CT images of the same healthy and ALI diseased pig. It shows poor correlation between estimated on healthy and ALI diseased pig EIT and CT images. 


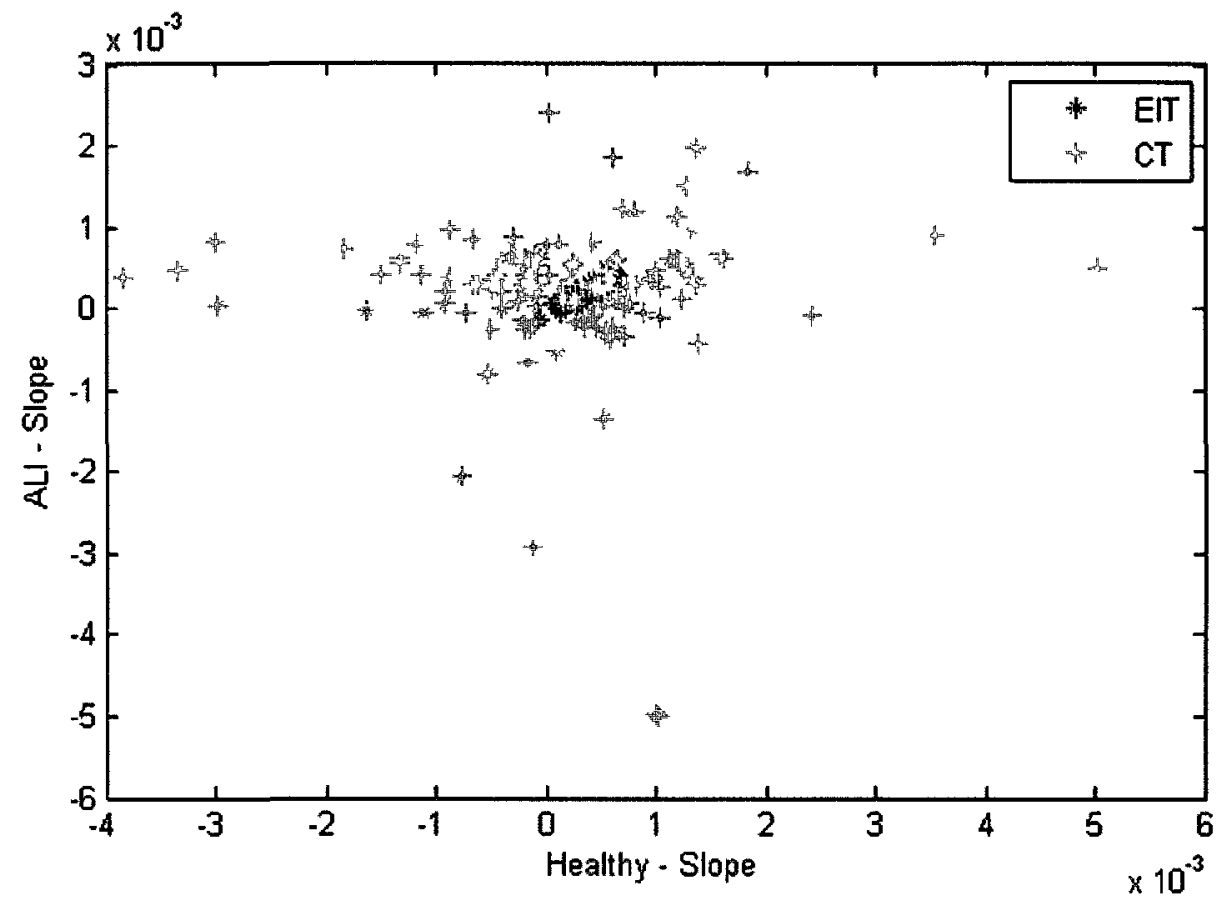

Figure 6-14: Correlation between research parameter Slope estimated on 130 ROIs selected on both EIT, blue "**", and CT ,green "*", images of healthy and ALI diseased pig labelled "ards22-03".

As seen in figure 6- 14, Slope research parameter was estimated on ROIs selected on EIT and CT images of healthy and ALI diseased pig. It shows positive correlation between the estimated slope on health and ALI diseased pig EIT images. Also, for the same parameter, it shows poor correlation between healthy and ALI diseased pig CT data.

The following table 6- 6 shows the correlation coefficients for the 5 research parameters between healthy and ALI diseased pig. Data shown in the table are from 4 pigs labelled "ards2203, 04, 05, and 08 " and for both EIT and CT images. 


\begin{tabular}{|c|c|c|c|c|c|c|}
\hline $\begin{array}{c}\text { Pig label } \\
\text { “ards22-" }\end{array}$ & EIT/CT & A1 & $\boldsymbol{\tau}_{1}$ & Slope & A2 & $\boldsymbol{\tau}_{2}$ \\
\hline \multirow{2}{*}{$\mathbf{0 3}$} & EIT & 0.928 & -0.180 & 0.854 & 0.958 & 0.371 \\
\cline { 2 - 7 } & CT & 0.306 & -0.032 & 0.007 & 0.484 & -0.074 \\
\hline \multirow{2}{*}{$\mathbf{0 4}$} & EIT & 0.989 & 0.558 & 0.495 & 0.970 & 0.726 \\
\cline { 2 - 7 } & CT & 0.949 & 0.553 & 0.799 & 0.968 & 0.712 \\
& EIT & 0.161 & -0.183 & 0.545 & 0.743 & 0.144 \\
\hline \multirow{2}{*}{08} & CT & 0.458 & 0.103 & 0.183 & 0.419 & -0.030 \\
\hline & EIT & 0.987 & -0.237 & 0.894 & 0.981 & -0.163 \\
\hline
\end{tabular}

Table 6- 6: Correlation coefficients for each research parameter between healthy and ALI diseased data for both EIT and CT images. Each parameter estimated on ROIs selected on image of healthy pig is compared to the same parameter estimated on the same image of the same ALI diseased pig. Pigs labelled "ards22-03, 04, 05, and 08" were analyzed for both EIT and CT images.

As seen in figure 6- 6, each research parameter estimated on ROIs selected on EIT and CT images of healthy pig are compared to the same parameter estimated ROIs selected on the same ALI diseased pig EIT and CT images. The table shows the correlation coefficient of each research parameter between healthy and ALI diseased pig images. For example, for pig labelled "ards22-03", there is a positive correlation when comparing A1 research parameter estimated on EIT images of healthy pig with the same parameter estimated on EIT images of the same ALI diseased pig.

Research parameters estimated from each ROI selected on EIT and CT images were studied and analyzed for both healthy and ALI pigs. Each parameter estimated on ALI EIT and CT images was subtracted from the same parameter estimated on healthy EIT and CT images. 
The result is divided by the healthy estimated value for the same parameter. The process is repeated to obtain a set of data for all ROIs selected on EIT and CT images. The average change and the standard deviation of the data set are obtained and presented in the following table 6- 7.

\begin{tabular}{|c|c|c|c|c|c|c|}
\hline $\begin{array}{c}\text { Pig } \\
\text { label } \\
\text { “ards2 } \\
-"\end{array}$ & $\begin{array}{c}\text { EIT/ } \\
\text { CT }\end{array}$ & A1 & $\boldsymbol{\tau}_{1}$ & Slope & A2 & $\boldsymbol{\tau}_{\mathbf{2}}$ \\
\hline \multirow{2}{*}{$\mathbf{0 3}$} & EIT & $-7.2 \% \pm 19.1 \%$ & $-80.1 \% \pm 26.2 \%$ & $43.7 \% \pm 41.1 \%$ & $-6.1 \% \pm 18.2 \%$ & $-24.1 \% \pm 9.0 \%$ \\
\cline { 2 - 7 } & CT & $-3.1 \% \pm 47.4 \%$ & $-18.2 \% \pm 88.6 \%$ & $-4.2 \% \pm 95.5 \%$ & $-1.2 \% \pm 44.1 \%$ & $-13.2 \% \pm 96.4 \%$ \\
\hline \multirow{2}{*}{$\mathbf{0 4}$} & EIT & $-6.2 \% \pm 16.1 \%$ & $-14.3 \% \pm 15.8 \%$ & $29.2 \% \pm 18.1 \%$ & $-5.3 \% \pm 18.2 \%$ & $12.2 \% \pm 6.9 \%$ \\
\cline { 2 - 7 } & CT & $1.0 \% \pm 10.3 \%$ & $-12.7 \% \pm 39.2 \%$ & $8.2 \% \pm 130 \%$ & $-1.9 \% \pm 11.4 \%$ & $-11.3 \% \pm 37.2 \%$ \\
\hline \multirow{2}{*}{$\mathbf{0 5}$} & EIT & $-42.2 \% \pm 130 \%$ & $-6.5 \% \pm 27.7 \%$ & $92.2 \% \pm 106 \%$ & $-80.1 \% \pm 209 \%$ & $-10.3 \% \pm 20.2 \%$ \\
\cline { 2 - 7 } & CT & $-2.2 \% \pm 40.2 \%$ & $-22.2 \% \pm 97.8 \%$ & $474 \% \pm 34.41 \%$ & $-6.1 \% \pm 52.5 \%$ & $-19.3 \% \pm 105 \%$ \\
\hline \multirow{2}{*}{$\mathbf{0 8}$} & EIT & $-7.1 \% \pm 0.21 \%$ & $-143 \% \pm 75.5 \%$ & $31.3 \% \pm 74.4 \%$ & $-13.2 \% \pm 31.8 \%$ & $-7.1 \% \pm 30.9 \%$ \\
\cline { 2 - 7 } & CT & $-5.7 \% \pm 42.2 \%$ & $4.2 \% \pm 84.3 \%$ & $28.7 \% \pm 669.1 \%$ & $-25.2 \% \pm 189 \%$ & $8.3 \% \pm 101 \%$ \\
\hline
\end{tabular}

Table 6- 7: the average change \pm the standard deviation of each parameter estimated on healthy and ALI images. Pigs labelled "ards22-03, 04, 05, and 08" were analyzed for both EIT and CT images.

As seen in table 6- 7, each parameter was studied and analyzed for both healthy and ALI EIT and CT ROIs for 4 pigs labelled "ards22-03, 04, 05, and 08". The table shows the average change for each parameter between healthy and ALI estimated values. For example, for pig labelled "ards22-03", A1 research parameter was analyzed for both healthy and ALI ROIs selected on EIT and CT images. The average change between healthy and ALI estimated values for all ROIs selected on EIT images is $-7.2 \%$ with a standard deviation equal to $19.1 \%$. 
Testing that healthy parameters are different from the same ALI parameters estimated on EIT and CT images was done by using the MATLAB 'ANOVA' function. It is an algorithm that compares the mean of healthy and ALI data and returns the p-value for the null hypothesis that the means of both data are equal. If the p-value is near zero, the null hypothesis may be rejected, indicating that the mean values of healthy and ALI animals are significantly different from each other.

\begin{tabular}{|c|c|c|c|c|c|c|}
\hline $\begin{array}{c}\text { Pig } \\
\text { label } \\
\text { "ards22 } \\
-"\end{array}$ & $\begin{array}{c}\text { EIT/ } \\
\text { CT }\end{array}$ & A1 & $\boldsymbol{\tau}_{1}$ & Slope & A2 & $\boldsymbol{\tau}_{2}$ \\
\hline \multirow{2}{*}{03} & EIT & 1 & 0 & 0 & 1 & 0 \\
\cline { 2 - 7 } & CT & 1 & 0.098 & 0.720 & 1 & 0.243 \\
\hline \multirow{204}{*}{0} & EIT & 1 & $2.52 \times 10^{-7}$ & $1.632 \times 10^{-4}$ & 1 & $5.10 \times 10^{-15}$ \\
\cline { 2 - 7 } & CT & 1 & 0.352 & 0.057 & 1 & 0.075 \\
\hline \multirow{2}{*}{05} & EIT & 1 & 0.241 & 0 & 1 & $7.77 \times 10^{-4}$ \\
\cline { 2 - 7 } & CT & 1 & 0.397 & $1.78 \times 10^{-10}$ & 1 & 0.011 \\
\hline \multirow{2}{*}{08} & EIT & 1 & 0.0007 & $3.73 \times 10^{-5}$ & 1 & 0.023 \\
\cline { 2 - 7 } & CT & 1 & $5.79 \times 10^{-7}$ & 0.685 & 1 & $1.87 \times 10^{-13}$ \\
\hline
\end{tabular}

Table 6- 8: P-value showing the null hypothesis that the means of both healthy and ALI parameters are equal. If $\mathrm{p}-$ value is close to zero, there is more doubt on the null hypothesis and shows that the mean of healthy parameters is different from the mean of ALI parameters.

As seen in table 6- 8, the p-value were calculated for each research parameter estimated on EIT and CT images. In this case, the null hypothesis cannot be rejected, and we cannot distinguish the means of healthy and ALI parameters. For example, for pig labelled "ards22-05", the p-value for $\tau_{2}$ parameter estimated on CT images was found to be 0.011 which indicates that the research parameter $\tau_{2}$ estimated on healthy CT images is different from the same parameter estimated on the ALI CT images. 
Testing the reliability of the correlation between healthy and ALI estimated research parameters was done by finding the p-value. The following table 6- 9 shows the p-value indicating the probability that the correlation between healthy and ALI parameters is not random.

\begin{tabular}{|c|c|c|c|c|c|c|}
\hline $\begin{array}{c}\text { Pig } \\
\text { label } \\
\text { ards22 } \\
-"\end{array}$ & $\begin{array}{c}\text { EIT/ } \\
\text { CT }\end{array}$ & A1 & $\boldsymbol{\tau}_{1}$ & Slope & A2 & $\boldsymbol{\tau}_{2}$ \\
\hline \multirow{2}{*}{03} & EIT & $8.02 \times 10^{-57}$ & 0.04 & $2.95 \times 10^{-38}$ & $2.40 \times 10^{-71}$ & $1.32 \times 10^{-5}$ \\
\cline { 2 - 7 } & CT & $3.84 \times 10^{-4}$ & 0.44 & 0.93 & $5.18 \times 10^{-9}$ & 0.39 \\
\hline \multirow{2}{*}{04} & EIT & $1.85 \times 10^{-73}$ & $1.88 \times 10^{-8}$ & $2.64 \times 10^{-81}$ & $2.92 \times 10^{-54}$ & $1.65 \times 10^{-15}$ \\
\cline { 2 - 7 } & CT & $1.34 \times 10^{-44}$ & $2.61 \times 10^{-8}$ & $3.89 \times 10^{-24}$ & $4.00 \times 10^{-53}$ & $9.64 \times 10^{-15}$ \\
\hline \multirow{2}{*}{05} & EIT & 0.46 & 0.15 & $4.32 \times 10^{-9}$ & 0.10 & 0.06 \\
\cline { 2 - 7 } & CT & $1.58 \times 10^{-6}$ & 0.30 & 0.06 & $1.42 \times 10^{-5}$ & 0.76 \\
\hline \multirow{2}{*}{08} & EIT & $2.84 \times 10^{-93}$ & 0.01 & $3.01 \times 10^{-41}$ & $2.41 \times 10^{-83}$ & 0.08 \\
\cline { 2 - 7 } & CT & $3.63 \times 10^{-9}$ & 0.67 & 0.78 & 0.25 & 0.62 \\
\hline
\end{tabular}

Table 6- 9: P-value for each parameter showing that the correlation between healthy and ALI parameters estimated on pigs labelled "ards22-03, 04, 05, and 08" for both EIT and CT images is not Random.

As seen in figure 6-9, p-value was calculated for each parameter estimated on EIT and CT images of healthy pigs labelled "ards $22-03,04,05$, and 08 ". The table shows that the correlation between healthy and ALI parameters is not random, indicating a high significant correlation between healthy and ALI data. 


\subsection{Excluded Pigs Results:}

EIT and CT images of 6 pigs were excluded from the analysis because of many reasons. For example, data from pig labelled "ards22-02" were excluded because there were n o A LI diseased CT images of the pig lungs.

Other pigs' results were excluded because data obtained from pig i.e. labelled "ards2201 " shows that the lungs get air filled at 2 stages and then air emptied from the lungs in 2 stages (figure 6-12). Therefore, data obtained from this pig is not suitable to estimate the 5 research parameters.

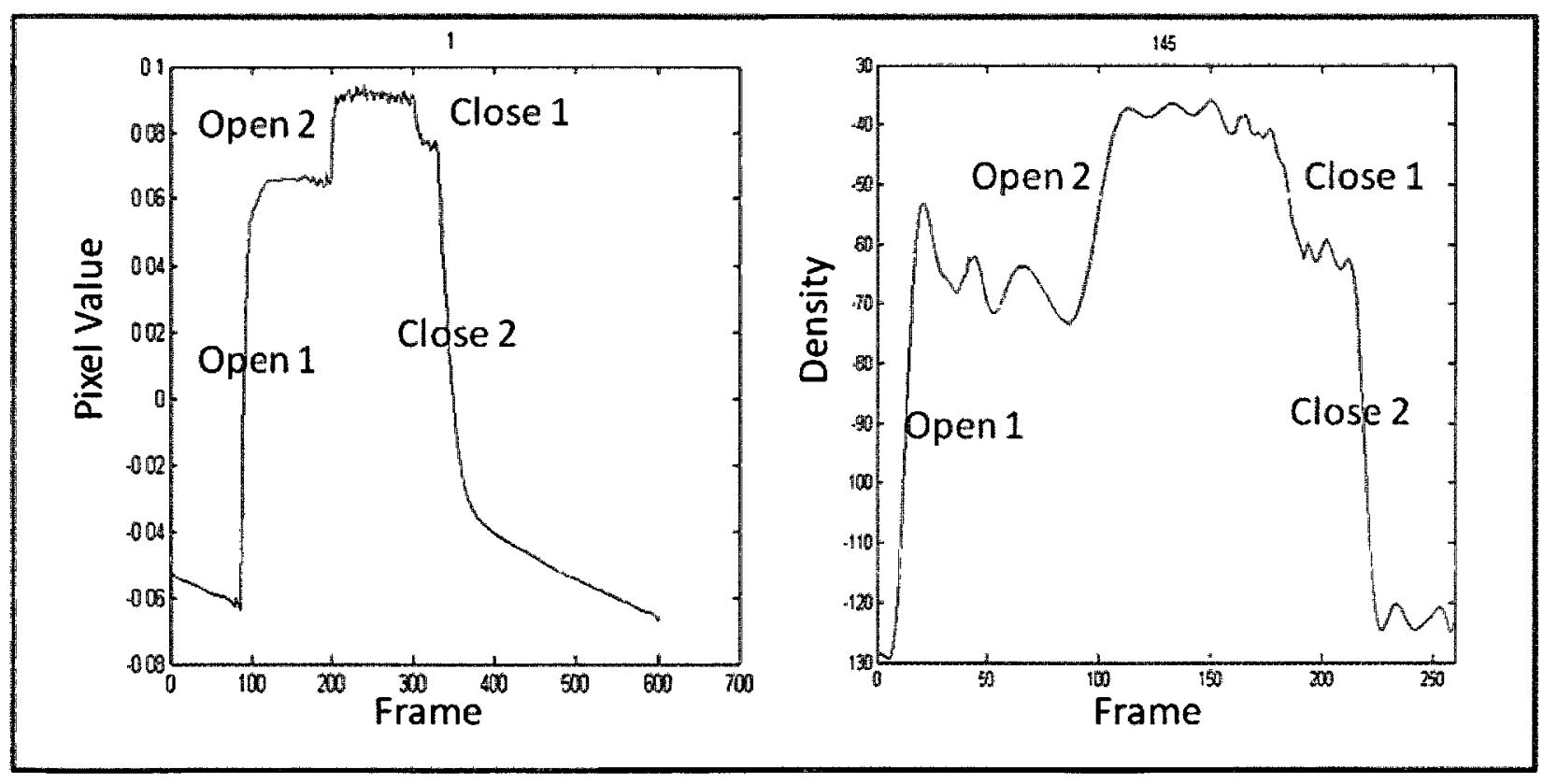

Figure 6- 15: (Left) pixel value versus frame number of a ROI selected on pig labelled "ards22-01" EIT image of the lungs. Right, density versus frame number of a ROI selected on the same pig CT image of the lungs. Both graphs show open 1 and open 2 indicating that the lungs open in 2 stages and air gets filled into the lungs. Close 1 and close 2 indicating that the lungs closes in 2 stages and air emptied from the lungs. 
As seen in figure 6-15, the left figure shows the pixel value versus frame number of a chosen ROI selected on EIT image of the lung for pig labelled "ards22-01". The right figure shows density versus frame number of a ROI selected on the CT image of the lung for the same pig.

Both graphs show that lungs get filled with air and the chosen ROI expands and opens at 2 stages (indicated by open 1 and open 2). Also, the figure shows that air gets emptied from the lungs, which in turn close at 2 stages (indicated by close 1 and close 2 ). 


\section{Chapter 7}

\section{Discussion:}

This chapter discusses the results presented in chapter 6. The research algorithm implemented validates ROIs selected on EIT images of pig's lungs using ROIs selected on dynamic CT images of the same pig's lungs. This technique studies and analyzes the functional behaviour of ROIs selected on sample pig's dynamic CT and EIT images of the lungs. Images of the 4 pigs labelled "ards $22-03,04,05$, and 08 " were studied and analyzed through this thesis algorithm before and after lung lavage (healthy and ALI diseased pigs).

Thesis algorithm estimates five research parameters on ROIs selected on both $\mathrm{CT}$ and EIT images of the lungs. The results show that the chosen ROIs selected on EIT and CT images of the lungs change as a result of air filling and emptying. The algorithm estimated the five research parameters for each chosen healthy and ALI diseased pig.

Research parameters A1 and A2 estimated on ROIs selected on the EIT image negatively correlate with the same parameters estimated on the CT image's ROIs. Parameters $\tau_{1}, \tau_{2}$, and the slope estimated on the EIT and CT images poorly correlate to each other. However, the 5 research parameters estimated in the CT images show a high positive correlation with $y$ coordinates of the ROIs selected on the image. On the other hand, there is a negative correlation when comparing the five research parameters estimated on the EIT image's ROIs with the ycoordinates of the chosen ROIs.

The negative correlation between image ROI amplitude values between EIT and CT was unexpected. Physiologically, ventilation is expected to increase in the dependent parts of the 
lungs (i.e., the dorsal region for a prone subject). This is consistent with the CT data in this thesis, but not with the EIT. However, EIT has been shown in many studies to show this dependent increase in ventilation (i.e., Frerichs et al, 2003). In order to attempt to explain why the opposite dependence is seen in this study, we note that the ROIs identified are relatively much lower in the EIT images than in the CT. Specifically, the upper lungs in the CT corresponds to the center or lower part of the ventilated region in the EIT images. Thus, as the ROI moves toward the dorsal region in the CT, it leaves the ventilated region in the EIT images, resulting in a decrease of ventilation. If this explanation is correct, the primary cause is the position error in the EIT images; ventilated regions are "stretched" away from their correct anatomical locations by errors in the EIT image reconstruction algorithm. In order to correct for this effect in future work, it is necessary to determine the pattern of such position errors (either from an appropriate model, or from the images) and to associate the appropriate ROIs in each modality.

Results show changes in research parameters estimated on healthy and ALI diseased pigs' images. Values of research parameter $\tau_{1}$ estimated on healthy pigs EIT and CT images are less than the estimated values on the ALI diseased pig's images. The A1 and A2 research parameters estimated on healthy pig EIT and CT images positively correlate to the same parameters estimated on EIT and CT images of the same ALI diseased pig. The Slope, $\tau_{1}$, and $\tau_{2}$ research parameters are either negatively or poorly correlated when comparing healthy pig EIT and CT images to the same ALI diseased pig EIT and CT images.

Thesis investigation shows a significant average change between each research parameter estimated on healthy and ALI diseased pigs' images. The standard deviation of the obtained data 
set was found very small (e.g., for the pig labelled "ards22-03", the standard deviation for A1 is 19.1\%). The estimated research parameters for both healthy and ALI diseased pigs' images tend to be very close to the average change obtained. It was found that there is a high hypothesis that there is difference between healthy and ALI parameters $\left(\tau_{1}\right.$ and $\tau_{2}$ ) for both EIT and CT images. These parameters poorly correlated when comparing healthy to ALI EIT and CT images. The correlation between healthy and ALI diseased pigs images is significant for some parameters such as $\mathrm{A} 1$, and $\mathrm{A} 1$. Other parameter such as $\tau_{1}$ and $\tau_{2}$ estimated on healthy images are less correlated to the same parameters estimated on the ALI EIT and CT images.

\subsection{Research Problems}

The main problem faced in this thesis project is matching the location of the ROIs selected on the EIT images with the location of the ROIs selected on the CT images. The locations of the ROIs are chosen to be on the lungs selected on both images. In case of the CT images, the lungs and other organs such as the heart are clear on the images. Therefore, the location of the chosen ROIs is certainly selected on the lungs. Whereas, the EIT images do not clearly show the location of the lungs or either any other organ which in turn lead to uncertain location of the ROIs selected on the lungs.

The way a pig placed in the CT machine affects on the shape of the CT image. For example, some CT images shows that the pig lying on its back and leaning on the left side. This position is clear in the image that the heart is covering the top area of the left lung and shows the right lung clearer and bigger than the left one (figure 7-1). 


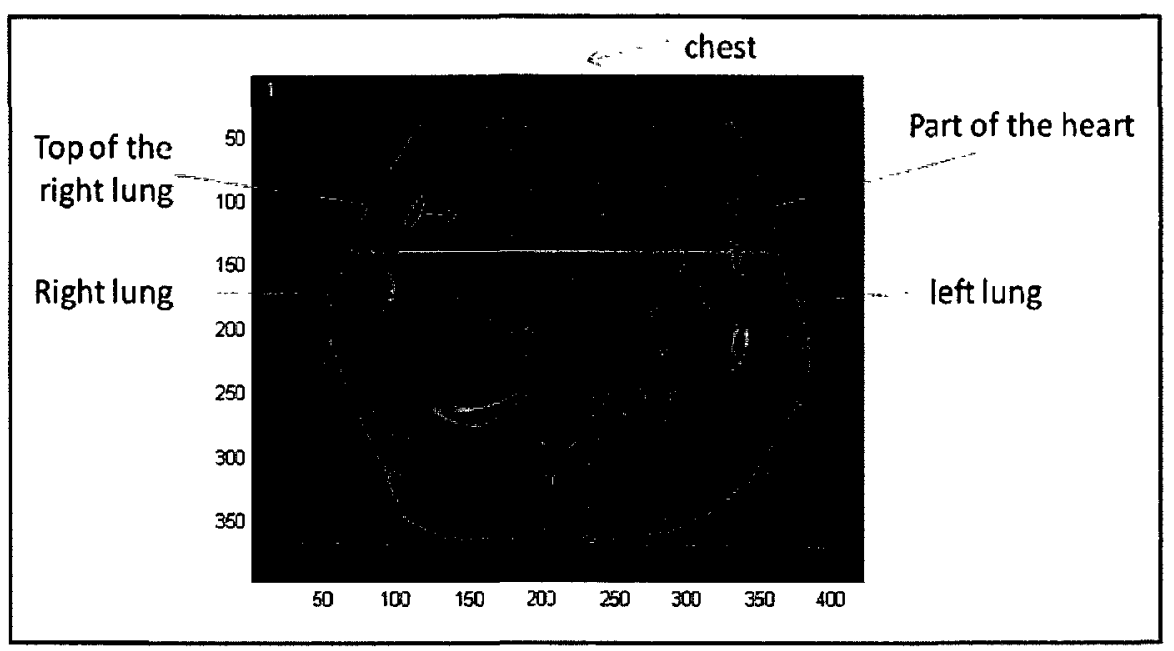

Figure 7- 1: pig's labelled "ards22-02" CT image of the lungs showing that the hear is coverring the top side of the left lung and the top boundary of the thorax doesn't show part of the chest.

As seen in figure 7-1, the top area of the right lung is clear in the CT image of the lungs and the heart covers the top left. This is not clear in the EIT images of the lungs and we cannot certainly locate the lungs or even the heart or any other organ. Also, in the CT image, the top boundary of the thorax is not showing part of the chest and there is extra space in the bottom of the image. EIT images do not show variations in the boundaries or the structure of any other organ. Therefore, the lungs size, shape and location are not clear and this lead to inaccuracy in choosing the ROIs on the EIT image (figure 7-2). 


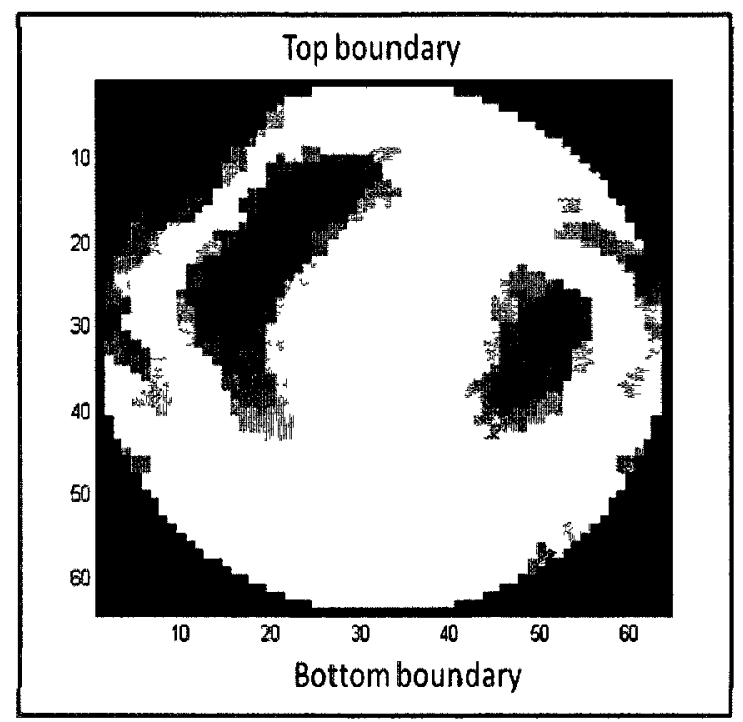

Figure 7- 2: pig labelled "ards22-02" EIT image of the lungs, thickness of the boundaries is not clear.

As seen in figure 7-2, EIT image of the same pig's lungs shows all the boundaries of the chest but with no difference between the top, left, right and bottom. The bottom boundary in the CT image shows the thickness of the pig's back, which is thicker than the top boundary, which shows the thickness of the chest. Looking at the EIT image, we can't determine the thickness of such a boundary, and can't determine the exact location of these organs.

The general shape of the EIT image is not the same shape as the CT image of the lungs. When the lungs expand during air filling, they get bigger, and when the lungs contract when emptying, it gets smaller. This is clear in the CT image we can detect the changes in the lungs size and the location of the boundaries or any other organ (please see figure 7-3). On the other hand, the EIT image does not show a change in the lungs size and the whole image stays at a fixed shape that is not changing as results of air filling or air emptying events (figure 7-3). 


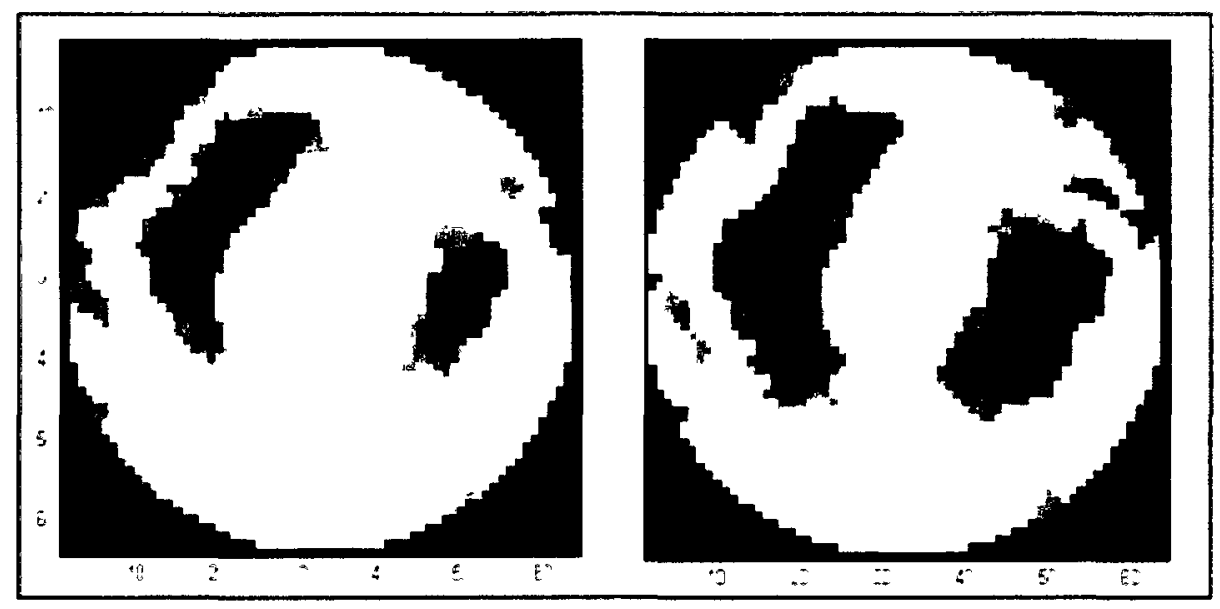

Figure 7- 3: (left) EIT image of the lungs for a healthy pig labelled "ards22-02" before air filling, (right) EIT image of the same pig's lungs after air filling. The size of both images is the same. Both images do not show a change in the shape or location of organs.

As seen in figure 7- 3, EIT image has the same shape and size in both left and right images. The left image shows the lungs before air filling and the right image shows the lungs after air filling. The size, shape, and location of lungs in both images are clear. The locations of the chest boundaries are the same before and after air filling.

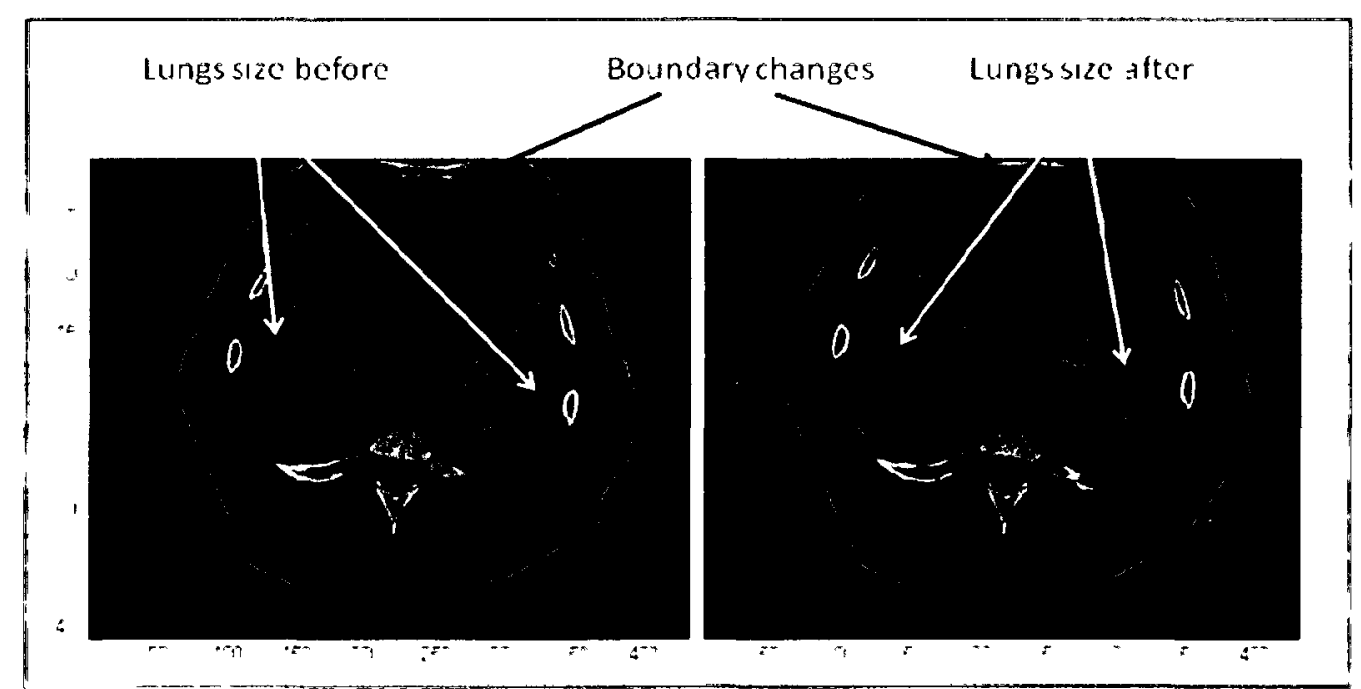

Figure 7- 4: (left) CT image of the lungs for healthy pig labelled "ards22-02" showing the size of the lungs and the boundaries before air filling, (right) CT image of the same pig's lungs showing the changes in the lungs size and the location of the boundaries. The lungs size and boundaries change before air filling (left image) and after air filling (right image). 
As seen in figure 7-4, CT image of the lungs changes in size as a result of air filling. The left image shows the lungs are smaller than $t$ he 1 ungs s hown in the $r$ ight $i$ mage. Also, the locations of the boundaries are changed in the right i mage comparing to the locations of the boundaries in the left image.

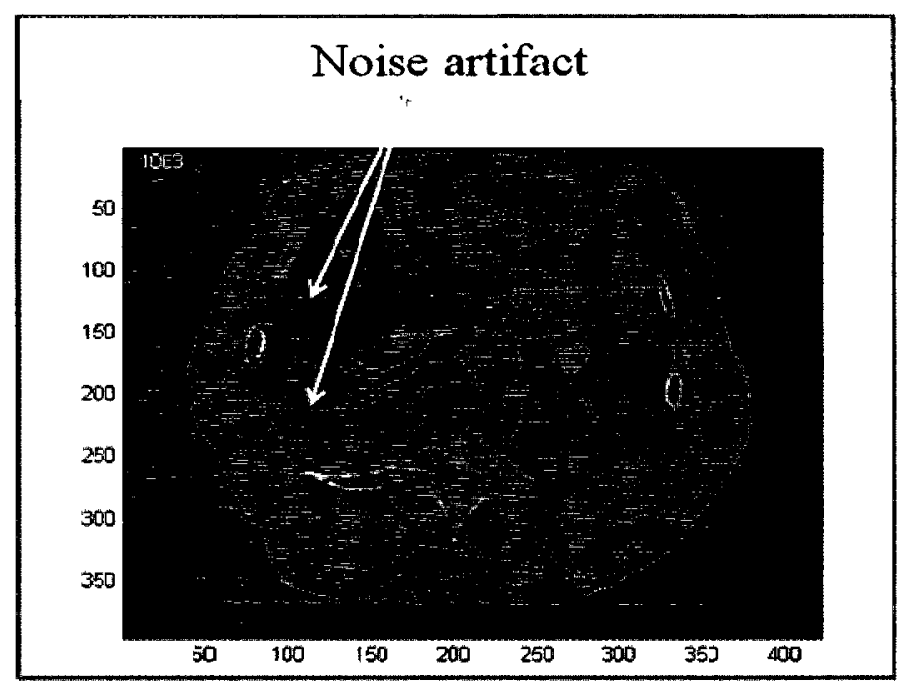

Figure 7- 5: CT image of ALI diseased pig labelled "ards22-02" showing noise artifacts (horizontal dashed lines) that affect the readings of ROIs and lead to a glitch in the graph obtained

As seen in figure 7- 5, a CT image of ALI diseased pig's lungs showing a noise artifact that distorts the image and results in an incorrect measurements of ROIs. The noise artifact is one of the problems that affect on the behaviour of each $\mathrm{R} O I$ and 1 ead to a n oise in the $\mathrm{g}$ raph obtained from all ROIs.

\section{$7.2 \quad$ Future work}

During the process of this research project, many interesting issues showed up. Some of these issues were problems faced in this thesis and some indicate promising points need to be 
resolved in order to improve the quality of EIT images. The following topics are important areas of the future work that might be accomplished in further research.

Clear organs shape detection. For example, the lung's shape is different than the heart shape. Therefore, EIT image reconstruction process should consider the actual shape of the lungs or any other organs in the body by separately modelling the changes in different areas of the image.

$>$ Organs motion tracking. For example, when the lungs expand and contract, it changes in shape and size and the boundaries of the chest change as well. EIT imaging technology could model the changes in size and motion happening inside the chest to the changes in size and location of the outer boundary (e.g., thorax and ribs).

$>$ Electrodes motion detection. The CT image shows the location of the electrodes on the boundaries of the thorax and when the lungs expand and contract, the location of the electrodes changes as a results of the ventilation process. EIT images should be considering this issue in order to minimize the number of errors (i.e., Gomez and Adler, 2008).

Studying and analyzing more ROIs selected on different areas on the heart, boundaries, and the air ways. This could help modeling the changes in breathing behaviour and monitor the volume of air being transferred through the lungs.

Analyzing EIT images time resolution by studying different ROIs considering the quantization effects. Interpolation technique can be implemented to mitigate this 
effect by improving the resolution of the time when analyzing different ROIs selected on EIT images. 


\section{References}

A. Adler and R. Guardo, "Electrical Impedance Tomography: Regularised Imaging and Contrast Detection", IEEE Trans. Medical Imaging, 15: 170-179, 1996.

A. Adler, R. Guardo, and Y. Berthiaume, "Imaging of Gastric Emptying with Electrical Impedance Tomography", 1994.

A. Adler and W.R.B. Lionheart. Uses and abuses of EIDORS: An extensible software base for EIT. Physiol. Meas., 27:S25-S42, 2006.

A. Adler, N. Shinozuka, Y. Berthiaume, R. Guardo, and J. Bates, "Electrical impedance tomography can monitor dynamic hyperinflation in dogs", J Appl Physiol 84:726-732, 1998.

American Lung Association, "Lung Disease Data: 2008", Acute Respiratory Distress Syndrome (ARDS): 9-13, 2008.

N. Applbaum and Y.h. Applbaum, "The Use of Medical Computed Tomography (CT) Imaging in the Study of Ceramic and Clay Archaeological Artifacts from the Ancient Near East", Springer, Netherlands, 2005.

V. Arora and U. Sankar. U, “Acute Lung Injury”, Clinical review, Lung India, No. 1 (P 3234), 1995.

B. C. Barber, "Electrical Impedance Tomography". The Biomedical Engineering Handbook: $2^{\text {nd }}$ Edition, CRC press LLC, 2000.

D. C. Barber and B. H. Brown, "Applied potential tomography", (Review Article), J. Phys. E: Sci. Instrum., 17:723-733, 1984.

D. C. Barber and B. H. Brown, "Electrical Impedance Tomography", British Technology Group Limited, 1997.

R. Bayford, P. Kantartzis, A. Tizzard, R. Yerworht, P. Liatsis, and A. Demosthenous, "Reconstruction Algoriths to Monitor Neonate Lung Function", Springer-Verlag Berlin Heidelberg, IFMBE Processins 17, pp. 352-255, 2007.

G. E. Bokinsky, "Comparision of measurements of total lung capacity by a computer-aided radiographic method with body plethysmography in a clinical setting", Martinus Nijhoff, International Journal of Clinical Monitoring and Computing 2: 163-166, 1986.

L. Borcea, "Electrical Impedance tomography", Institute of Physics Publishing, Inverse Problem 18, F99-R136, 2002 
B. Bouhemad, J. Richecoeur, Q. Lu, L. M. Malbouisson, P. Cluzel. J. Rouby, and ARDS CT Scan Study Group, "Effective of contrast material on computed tomographic measurements of lung volumes in patients with acute lung injury", Critical Care, 7:63-71, 2002.

D.J. Brenner and E.J. Hall, "Computed Tomography - An Increasing Source of Radiation Exposure", Review Article, the New England Journal of Medicine, 357:2277-84, 2007.

D. S. Calfee, "Acute Lung Injury/ARDS Update", American Thoracic Society, 2009.

V. Chan, J. Pisegna, R. Rosian, and S. E. DiCarlo, "Model Demonstrating Respiratory Mechanics for High School Students", The American Physiological Society, Volume 15, Number 1, 1996.

T. Dai, and A. Adler, "Electrical Impedance Tomography Reconstruction Using L1 Norms Data and images", $30^{\text {th }}$ Annual international IEEE EMBS Conference, 2008.

M. David, J. Karmrodt, C. Bletz, S. David, A. Herweling, H. Kauczor, and K. Markstaller, "Analysis of Atelectasis, Ventilated, and Hyperinflated Lung During Mechanical Ventialtion by Dynamic CT", American College of Chest Physicians, 128, 5, 3757-3770, 2005.

J. Fagon and J. Chastre,"Diagnosis and treatment of nosocomial pneumonia in ALI/ARDS patients", European Respiratory Journal, 22 supple 42, 77s-83s, 2003.

I. Frerichs, G. Hahn, T. Schröder and G. Heilige, "Electrical impedance tomography in monitoring experimental lung injury", Intensive Care Medicine, Springer-Verlag, 24:829836, 1998.

I. Frerichs, G. Hahn, and G. Hellige, "Thoracic electrical impedance tomographic measurements during volume controlled ventilation-effects of tidal volume and positive endexpiratory pressure", IEEE Transactions on Medical Imaging, Vol. 18. No. 9. 1999.

I. Frerichs, H. Schiffmann, R. Oehler, T. Dudykevych, G. Hahn, J. Hinz, G. Hellige, "Distribution of lung ventilation in spontaneously breathing neonates lying in different body positions", Intensive Care Medicine, Springer-Verlag, 29:787-794, 2003.

L. Gattinoni, P. Caironi, M. Cressoni, D. Chiumello, M. Ranieri, M. Quintel, S. Russo, N. Patroniti, R. Cornejo, G. Bugedo, "Lung Recruitment in patients with the Acute Respiratory Distress Syndrome", N Engl J Med, 354: 1775-1786, 2006.

L. Geddes and R. Roeder, "Handbook of Electrical hazards and Accidents", Second Edition. Lawyers and Judges Publishing Company, Inc, 2006 
C. Gómez-Laberge and A. Adler, "Direct EIT Jacobian calculations for conductivity change and electrode movement", Physiological Measurement, Volume 29, S89-S99, 2008.

A. Hartinger, H. Gagnon, and R. Guardo, "Accounting for hardware imperfections in EIT image reconstruction algorithms", Physiological Measurement, Volume 28, Number 7, 2007.

H. Hayashi, M. Machida, T. Sekine, H. Yamaguchi, T. Kiriyama, "Beam-hardening artifacts on computed tomography images caused by lanthanum carbonate hydrate in a patient on dialysis", Japan Radiological Society, 28:322-324, 2010.

Health Canada, "Health Effects of Air pollution", [Online] visited on (July, 2010), Available: http://www.hc-sc.gc.ca/ewh-semt/air/out-ext/effe/health_effects-effets_sante-eng.php

HealthCare Human Factors Group, "Computed Tomography Radiation Safety Issues in Ontario", University Health Network, 2006

D. S. Holder, "Electrical Impedance Tomography (EIT) of Brain function", Brain Topography, Volume 5, Number 2, 1992.

Inner health group, “LUNGS FACTROS”, Michael's Naturopathic Programs, issue \# 10280, 1996.

X. Li, J. Ni, T. He, G. Wang, S. Wang, and B. Knosp, "Parallel Iterative CT Image Reconstruction on a Linux Cluster of Legacy Computers", University of Iowa, 370-373, 2005

W. R.B. Lionheart, F.J. Lidgey, C. N. Mcleod, K.S. Paulson, M.K. Pidcock, and Y. Shi, "Electrical Impedance Tomography for high speed chest imaging", The University of Manchester, MIMS EPrint, 1998.

M. A. Matthay, G. A. Zimmerman, C. Esmon, J. Bhattacharya, B. Coller, C. M. Doerschuk, J. Floros, M. A. Gimbrone Jr, E. Hoffman, R. D. Hubmayr, M. Leppert, S. Matalon, R. Munford, P. Parsons, A. S. Slutsky, K. J. Tracey, P. Ward, D. B. Gail, and A. L. Harabin, "Furure Research Dictionary in Acute Lung Injury NHLBI Workshop Summary", Am J Respir Crit Care Med vol.167, pp 1027-1035, 2003.

N. Moreno, B. Z. Tharp, and J. Dresden, "Breathing Machine", Baylor College of Medicine, 2006.

A. Movafeghi, A. Nateghi, M. Soleimani, M. Kargarnovin, and H. Soltanianzadeh. "Image Reconstruction Algorithms or Sut-1 EIT System", 2004 
R. Nanchal, E. J. Jimenez, and F. E. Poalillo, “ALI, ARDS, and Protective Lung Ventilation", Intensive and Critical Care Medicine, Springer-Verlag Italia, 18: 207- 218, 2009.

U.S. Navy Diving Manual,“Underwater Physiology and Diving Disorders”, Revision 6, Volume 1, Ch. 3, pages 6-12, SS521-AG-PRO-010, 2008.

P. Neligan, "Pulmonary Critical care Medicine Tutorials", University of Pennsylvania, 2002, [Online], visited on (July 2010), Available: http://www.ccmtutorials.com/

R. Notter, "Lung Surfactants Basic Science and Clinical Applications", vol. 149, (Lung Biology in Health and Diseases), New York, N. Y, Marcel Dekker, 2000.

N. Otsu, "A Threshold Selection Method from Gray-Level Histograms", IEEE transactions on Systems, Man, and Cybernetics, vol. 9, no. 1, pp. 62-66, 1979.

A.S. Pandya, A. Arimoto, A. Agarwal, and Y. Kinoushi, "A Novel Approach for Measuring Electrical Impedance Tomography for Local Tissue with Artificial Intelligent Algorithm", International Journal of Biometrics and Bioinformatic. (IJBB), Volume (3), Issue (5), 2009.

D. Pointer, "Computed Tomography (CT) Scan Image Reconstruction on the SRC-7", SRC Computers, Inc, 2008

C. Putensen, J. Zinserling, and H. Wrigge, "Electrical Impedance Tomography for monitoring of Regional Ventilation in Critical III Patients", Springer Berlin Heidelberg, Volume 2006, Part 10, 448-457, 2006.

Radiological Society of North America, "Computed Tomography (CT) - Children (Pediatric)", 2010.

Rensselaer Polytechnic Institute, Electrical Impedance Imaging Laboratory [Online], visited on (July 2010), Available: http://www.ecse.rpi.edu/homepages/saulnier/eit/eit.html

A. T. Rotta, and D. M. Steinhorn, "Conventional mechanical ventilation in pediatrics", Review Article, jornal de Pediatria, vol. 83 no.2, 2007.

W. Ruan, R. Guardo, and A. Adler, "Experimental Evaluation of Two Iterative Reconstruction Method for Induced Current Electrical Impedance Tomography", IEEE Transaction on Medical imaging, VOL 15, NO. 2, 1996

I. Schneider, R. Kleffel, D. Jennings, and A. Courtenay, "Design of an electrical impedance tomography phantom using active elements", Medical \& Biological Engineering \& Computing, VOL 38, 390-394, 2000.

R. Stacey, "Electrical Impedance Tomography", Department of Energy and by the Department of Petroleum Engineering, Stanford University, 2006 
TutorVista Global Pvt. Ltd, "Parts and Functions of Respiratory System", [Online] visited on (August, 2010), Available: http://www.tutorvista.com/biology/parts-and-functions-ofrespiratory-system.

J. Wanger, J.L. Clausen, A. Coates, O.F. Pedersen, V. Brusasco, F. Burgos, R. Casaburi, R. Crapo, P. Enright, C.P.M. van der Grinten, P. Gustafsson, J. Hankinson, R. Jensen, D. Johnson, N. MacIntyre, R. McKay, M.R. Miller, D. Navajas, R. Pellegrino and G. Viegi, "Standardisation of the measurements of lung volumes", European Respiratory Journal, 26:511-522, 2005.

B. Xiaoyan, W. Huaxiang, Z. Bo, and S. Xiaolei, "Lung Ventilation Functional Monitoring Based on Electrical Impedance Tomography", Transactions of Tianjin University, vol.15 No. 1, pages 7-12, 2009. 